ESAIM: M2AN 55 (2021) S811-S851

https://doi.org/10.1051/m2an/2020058
ESAIM: Mathematical Modelling and Numerical Analysis

www.esaim-m2an.org

\title{
AN ASYMPTOTICALLY COMPATIBLE APPROACH FOR NEUMANN-TYPE BOUNDARY CONDITION ON NONLOCAL PROBLEMS
}

\author{
Huaiqian You ${ }^{1}$, Xin Yang Lu², Nathaniel Trask ${ }^{3}$ and Yue Yu ${ }^{1, *}$
}

\begin{abstract}
In this paper we consider 2D nonlocal diffusion models with a finite nonlocal horizon parameter $\delta$ characterizing the range of nonlocal interactions, and consider the treatment of Neumannlike boundary conditions that have proven challenging for discretizations of nonlocal models. We propose a new generalization of classical local Neumann conditions by converting the local flux to a correction term in the nonlocal model, which provides an estimate for the nonlocal interactions of each point with points outside the domain. While existing 2D nonlocal flux boundary conditions have been shown to exhibit at most first order convergence to the local counter part as $\delta \rightarrow 0$, the proposed Neumanntype boundary formulation recovers the local case as $O\left(\delta^{2}\right)$ in the $L^{\infty}(\Omega)$ norm, which is optimal considering the $O\left(\delta^{2}\right)$ convergence of the nonlocal equation to its local limit away from the boundary. We analyze the application of this new boundary treatment to the nonlocal diffusion problem, and present conditions under which the solution of the nonlocal boundary value problem converges to the solution of the corresponding local Neumann problem as the horizon is reduced. To demonstrate the applicability of this nonlocal flux boundary condition to more complicated scenarios, we extend the approach to less regular domains, numerically verifying that we preserve second-order convergence for non-convex domains with corners. Based on the new formulation for nonlocal boundary condition, we develop an asymptotically compatible meshfree discretization, obtaining a solution to the nonlocal diffusion equation with mixed boundary conditions that converges with $O\left(\delta^{2}\right)$ convergence.
\end{abstract}

Mathematics Subject Classification. 45K05, 76R50, 65R20, 65G99.

Received March 15, 2019. Accepted December 6, 2019.

\section{BACKGROUND}

In recent years, there has been great interest in using nonlocal integro-differential equations (IDEs) as a means to describe physical systems, due to their natural ability to describe physical phenomena at small scales and their reduced regularity requirements which lead to greater flexibility $[3,9,15-17,20,21,25$, $26,29,31,34-36,42,47,48,55,58,60,69,70]$. In particular, nonlocal problems with Neumann-type boundary constraints have received particular attention $[1,7,8,18,19,23,27,28,30,32,39,41,52,53,57,61,69]$ due to their

Keywords and phrases. Integro-differential equations, nonlocal diffusion, Neumann-type boundary condition, meshless, asymptotic compatibility.

1 Department of Mathematics, Lehigh University, 14 East Packer Avenue, Bethlehem, PA 18015, USA.

2 Department of Mathematical Sciences, Lakehead University, 955 Oliver Road, Thunder Bay, ON P7B 5E1, Canada.

3 Center for Computing Research, Sandia National Laboratories, 1450 Innovation Parkway, Albuquerque, NM 87123, USA.

*Corresponding author: yuy214@lehigh.edu 
prevalence in describing problems related to: interfaces [2], free boundaries, and multiscale/multiphysics coupling problems $[5,6,43,59,68]$. Unlike classical PDE models, in the nonlocal IDEs the boundary conditions must be defined on a region with non-zero volume outside the surface $[19,28,61]$, in contrast to more traditional engineering scenarios where boundary conditions are typically imposed on a sharp co-dimension one surface. Therefore, theoretical and numerical challenges arise from how to mathematically impose inhomogeneous Neumann-type boundary conditions properly in the nonlocal model. For instance, in the peridynamic theory of solid mechanics $[4,22,33,37,38,44-46,60,62,65,67]$, the classical description of material deformation locally via a deformation gradient is replaced by a nonlocal interaction described with integral operators. In these models, it has been shown that the careless imposition of traction conditions on the nonlocal boundary induces an unphysical strain energy concentration, leading in turn to the material being softer near the boundary. Such artificial phenomena are referred to in the literature as a "surface" or "skin" effect $[11,40]$. On the other hand, differs from the local problems, in some nonlocal problems boundary effects play a major role. For example, in nonlocal minimal surface problems, the "stickiness" effect arises and the boundary datum may not be attained continuously $[12,24]$. All the above examples indicate that careful treatments of the nonlocal boundary conditions are critical for the study of nonlocal problems.

A key feature in the discretization of nonlocal models has been the concept of asymptotic compatibility, originally introduced by Tian and Du [63], which describes the ability of a nonlocal discretization to recover a corresponding local model as both $\delta$ and a characteristic discretization lengthscale are reduced at the same rate. We advocate the development of both nonlocal boundary treatment and discretization with the objective of preserving this limit. In doing so, we ensure that nonlocal models recover a well-understood classical limit, avoiding phenomena such as the surface effect. To this end, we introduce here a non-local boundary treatment that is designed to recover the classical theory. After rigorously proving that this nonlocal boundary value problem recovers the desired local Neumann problem as $\delta \rightarrow 0$, we have a firm mathematical foundation upon which to demonstrate asymptotic compatibility, where we will develop an asymptotically compatible numerical method and demonstrate its high-order convergence and a lack of artificial surface phenomena.

In this paper, we study compactly supported nonlocal integro-differential equations (IDEs) with radial kernels. For concreteness, we focus on the nonlocal diffusion equation

$$
L_{\delta} u_{\delta}:=-2 \int_{B(\mathbf{x}, \delta)} J_{\delta}(|\mathbf{x}-\mathbf{y}|)\left(u_{\delta}(\mathbf{y})-u_{\delta}(\mathbf{x})\right) \mathrm{d} \mathbf{y}=f(\mathbf{x}), \quad \mathbf{x} \in \Omega \subset \mathbb{R}^{N},
$$

although the proposed technique is applicable to more general problems. Here $B(\mathbf{x}, \delta)$ is the ball centered at $\mathbf{x}$ with radius $\delta, u_{\delta}(\mathbf{x})$ is the solution, $\Omega$ is a bounded and connected domain in $\mathbb{R}^{N}(N=2), f(\mathbf{x})$ is given data, and the kernel function $J_{\delta}: \mathbb{R} \rightarrow \mathbb{R}$ is parameterized by a positive horizon parameter $\delta$ which measures the extent of nonlocal interaction. We further take a popular choice of $J_{\delta}$ as a rescaled kernel given by

$$
J_{\delta}(|\boldsymbol{\xi}|)=\frac{c}{\delta^{N+2}} J\left(\frac{|\boldsymbol{\xi}|}{\delta}\right),
$$

where $J:[0, \infty) \rightarrow \mathbb{R}$ is a nonnegative and continuous function with $\int_{\mathbb{R}^{N}} J(|\mathbf{z}|)|\mathbf{z}|^{2} \mathrm{~d} \mathbf{z}=N$. Similar as in [61], we also assume that $J(r)$ is nonincreasing in $r$, strictly positive in $r \in[0,1]$ and vanishes when $r>1$. In this work we aim to design a new formulation of Neumann-type constraint for the nonlocal problem (1.1) with mixed boundary conditions of Dirichlet, Neumann and mixed type, and present a numerical discretization of the resulting problem.

We pose three requirements for this formulation:

(1) The constraint should be a proper nonlocal analogue to the local Neumann-type boundary conditions, so the formulation provides an approximation of physical boundary conditions on a sharp surface.

(2) A boundary value problem given by the nonlocal Neumann-type constraint with the nonlocal diffusion equation (1.1) should be well-posed. Rigorous mathematical analysis on the existence, uniqueness and continuous dependence on data should be addressed for the associated variational problem. 

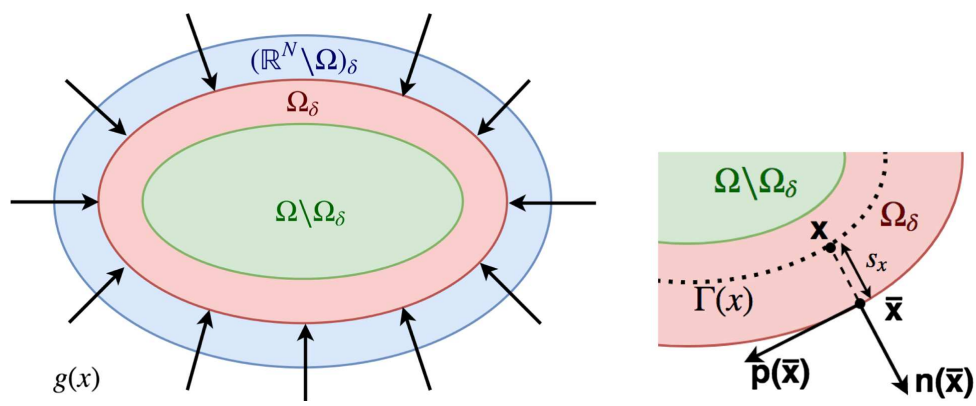

FIgURE 1. Left: notations for the domain, where $\Omega$ is represented by the green and red regions together, and the nonlocal Neumann boundary condition is applied on the red region $\Omega_{\delta}$. Right: notations for the projection of point $\mathbf{x} \in \Omega_{\delta}$, the corresponding unit tangential vector $\mathbf{p}(\overline{\mathbf{x}})$ and the unit normal vector $\mathbf{n}(\overline{\mathbf{x}})$.

(3) The nonlocal Neumann-type boundary value problem should recover the classical Neumann problem as $\delta \rightarrow 0$, preferably with an optimal convergence rate of $O\left(\delta^{2}\right)$ in the $L^{\infty}$ norm.

In the first part of the paper, we provide analysis of the boundary value problem (BVP) to establish the consistency and well-posedness of the boundary value problem. We establish here second-order convergence on non-trivial geometry, improving upon the first-order, one-dimensional analysis found in the literature.[19,30,61]. In the second part of this paper, we will present a new asymptotically compatible meshfree discretization of the proposed nonlocal BVP [10,54,64]. We pursue an extension of previous work by Trask et al. [64] utilizing an optimization-based approach to meshfree quadrature. This framework is attractive due to its demonstrated ability to achieve high-order asymptotically compatible solutions on unstructured data, which is complementary to the objective of developing boundary conditions consistent for irregular geometries. By introducing the new boundary treatment we will demonstrate improved second-order convergence over the previously demonstrated first order-convergence shown for Neumann problems [64].

The paper is organized as follows. We first present in Section 2 a definition of the nonlocal Neumann-type boundary condition and the corresponding nonlocal variational problem, together with the associated nonlocal operator and natural energy space. In Section 3, we study the well-posedness of the nonlocal variational problem for convex and sufficiently regular domains. We provide a consistency result for the nonlocal BVP by showing that the weak solution of the proposed nonlocal Neumann-type constrained value problem (denoted as $u_{\delta}$ ) converges to the solution of the corresponding classical diffusion problem (denoted as $u_{0}$ ) as the interaction horizon $\delta \rightarrow 0$ in the $L^{2}(\Omega)$ norm. Although the main proof in this section has assumed homogeneous Neumann boundary conditions, a discussion on the extension to inhomogeneous Neumann-type boundary condition is also provided. Furthermore, in Section 4 we prove the $O\left(\delta^{2}\right)$ convergence rate of the continuous nonlocal solution $u_{\delta}$ to $u_{0}$ in the $L^{\infty}(\Omega)$ norm without extra regularity assumptions on $u_{\delta}$. Numerical results utilizing a meshfree quadrature rule are presented in Section 5. To verify the asymptotic compatibility of the combined boundary treatment and the meshfree numerical scheme, in Section 6 we use manufactured solutions to demonstrate the convergence of the discrete model to the local solution as both the discretization length scale $h$ and the nonlocal interaction length scale $\delta \rightarrow 0$. Furthermore, in Section 7 we extend the approach to domains with corners which indicate that the conclusions of the model problem and the convergence rates extrapolate to nontrivial problems of interest to the broader engineering community. Section 8 summarizes our findings and discusses future research. The appendices include additional technical details on the theoretical analysis. 


\section{A NONLOCAL FLUX CONDition FOR 2D DIFFUSION PROBLEM}

In this section, we first introduce a nonlocal flux boundary condition, and then provide a corresponding nonlocal variational problem along with the associated energy space for the purpose of analysis. Given that $\Omega \in \mathbb{R}^{N}(N=2)$ is a bounded, convex, connected and $C^{3}$ domain, we seek a nonlocal analogue to the local Neumann boundary condition $\frac{\partial u}{\partial \mathbf{n}}=g(\mathbf{x}), \mathbf{x} \in \partial \Omega$ in the following classical problem

$$
\begin{cases}L_{0} u_{0}:=-\triangle u_{0}=f(\mathbf{x}), & \text { in } \Omega \\ \frac{\partial u_{0}}{\partial \mathbf{n}}=g(\mathbf{x}), & \text { on } \partial \Omega \\ \int_{\Omega} u_{0}(\mathbf{x}) \mathrm{d} \mathbf{x}=0 . & \end{cases}
$$

Here $\mathbf{n}(\mathbf{x})$ is the unit exterior normal to $\Omega$ at $\mathbf{x}$. Moreover, we will use $\mathbf{p}(\mathbf{x})$ to represent the unit tangential vector with orientation clockwise to $\mathbf{n}(\mathbf{x})$. Before introducing our nonlocal formulation, we denote the following notation (see Fig. 1 for illustration)

$$
\Omega_{\delta}:=\{\mathbf{x} \in \Omega \mid \operatorname{dist}(\mathbf{x}, \partial \Omega)<\delta\}, \quad\left(\mathbb{R}^{N} \backslash \Omega\right)_{\delta}:=\left\{\mathbf{x} \in \mathbb{R}^{N} \backslash \Omega \mid \operatorname{dist}(\mathbf{x}, \partial \Omega)<\delta\right\} .
$$

We further assume sufficient regularity in the boundary that we may take $\delta$ sufficiently small so that for any $\mathbf{x} \in \Omega_{\delta}$, there exists a unique orthogonal projection of $\mathbf{x}$ onto $\partial \Omega$. We denote this projection as $\overline{\mathbf{x}}$. Therefore, one has $\overline{\mathbf{x}}-\mathbf{x}=s_{x} \mathbf{n}(\overline{\mathbf{x}})$ for $\mathbf{x} \in \Omega_{\delta}$, where $0<s_{x}<\delta$. We also assume that for $\mathbf{x} \in \Omega_{\delta}$, we can find a contour $\Gamma(\mathbf{x})$ which is parallel to $\partial \Omega$. In the following contents, we denote $\mathbf{x}_{l}$ as the point with distance $l$ to $\mathbf{x}$ along $\Gamma(\mathbf{x})$ following the $\mathbf{p}(\overline{\mathbf{x}})$ direction, and $\mathbf{x}_{-l}$ as the point with distance $l$ to $\mathbf{x}$ in the opposite direction. Moreover, we employ the following notations for the directional components of the Hessian matrix of a scalar function $v$ :

$$
[v(\mathbf{x})]_{p p}:=\mathbf{p}^{T}(\overline{\mathbf{x}}) \nabla^{2} v(\mathbf{x}) \mathbf{p}(\overline{\mathbf{x}}), \quad[v(\mathbf{x})]_{n n}:=\mathbf{n}^{T}(\overline{\mathbf{x}}) \nabla^{2} v(\mathbf{x}) \mathbf{n}(\overline{\mathbf{x}}), \quad[v(\mathbf{x})]_{p n}:=\mathbf{p}^{T}(\overline{\mathbf{x}}) \nabla^{2} v(\mathbf{x}) \mathbf{n}(\overline{\mathbf{x}}),
$$

and the higher order derivative components are similarly defined.

Since $B(\mathbf{x}, \delta) \cap\left(\mathbb{R}^{N} \backslash \Omega\right)_{\delta} \neq \emptyset$ for $\mathbf{x} \in \Omega_{\delta}$, from (1.1) we have

$$
\begin{aligned}
L_{\delta} u_{\delta} & =-2 \int_{B(\mathbf{x}, \delta)} J_{\delta}(|\mathbf{x}-\mathbf{y}|)\left(u_{\delta}(\mathbf{y})-u_{\delta}(\mathbf{x})\right) \mathrm{d} \mathbf{y} \\
& =-2 \int_{B(\mathbf{x}, \delta) \cap \Omega} J_{\delta}(|\mathbf{x}-\mathbf{y}|)\left(u_{\delta}(\mathbf{y})-u_{\delta}(\mathbf{x})\right) \mathrm{d} \mathbf{y}-2 \int_{B(\mathbf{x}, \delta) \cap\left(\mathbb{R}^{N} \backslash \Omega\right)_{\delta}} J_{\delta}(|\mathbf{x}-\mathbf{y}|)\left(u_{\delta}(\mathbf{y})-u_{\delta}(\mathbf{x})\right) \mathrm{d} \mathbf{y},
\end{aligned}
$$

hence we need to approximate the integral in $B(\mathbf{x}, \delta) \cap\left(\mathbb{R}^{N} \backslash \Omega\right)_{\delta}$ and obtain a formulation with correction terms. Specifically, we propose the following flux boundary condition for (1.1): for $\mathbf{x} \in \Omega_{\delta}$

$$
\begin{aligned}
& -2 \int_{\Omega} J_{\delta}(|\mathbf{x}-\mathbf{y}|)\left(u_{\delta}(\mathbf{y})-u_{\delta}(\mathbf{x})\right) \mathrm{d} \mathbf{y}-\int_{\mathbb{R}^{N} \backslash \Omega} J_{\delta}(|\mathbf{x}-\mathbf{y}|)(\mathbf{y}-\mathbf{x}) \cdot \mathbf{n}(\overline{\mathbf{x}})(g(\mathbf{x})+g(\mathbf{y})) \mathrm{d} \mathbf{y} \\
& -\int_{\mathbb{R}^{N} \backslash \Omega} J_{\delta}(|\mathbf{x}-\mathbf{y}|)|(\mathbf{y}-\mathbf{x}) \cdot \mathbf{p}(\overline{\mathbf{x}})|^{2} \mathrm{~d} \mathbf{y}\left[u_{\delta}(\mathbf{x})\right]_{p p}=f(\mathbf{x}),
\end{aligned}
$$

where the second and third terms aim to provide an approximation for

$$
-2 \int_{\mathbb{R}^{N} \backslash \Omega} J_{\delta}(|\mathbf{x}-\mathbf{y}|)\left(u_{\delta}(\mathbf{y})-u_{\delta}(\mathbf{x})\right) \mathrm{d} \mathbf{y} .
$$

Since the boundary condition $g(\mathbf{x})$ is defined only on $\partial \Omega$, the $g(\mathbf{x})$ and $g(\mathbf{y})$ terms in (2.2) will be approximated with the following (local) extensions

$$
\begin{aligned}
& g(\mathbf{x}) \approx g(\overline{\mathbf{x}})-(\mathbf{x}-\overline{\mathbf{x}}) \cdot \mathbf{n}(\overline{\mathbf{x}}) f(\mathbf{x})-(\mathbf{x}-\overline{\mathbf{x}}) \cdot \mathbf{n}(\overline{\mathbf{x}})\left[u_{\delta}(\mathbf{x})\right]_{p p}, \\
& g(\mathbf{y}) \approx g(\overline{\mathbf{x}})-(\mathbf{y}-\overline{\mathbf{x}}) \cdot \mathbf{n}(\overline{\mathbf{x}}) f(\mathbf{x})-(\mathbf{y}-\overline{\mathbf{x}}) \cdot \mathbf{n}(\overline{\mathbf{x}})\left[u_{\delta}(\mathbf{x})\right]_{p p} .
\end{aligned}
$$


Furthermore, we replace $\left[u_{\delta}(\mathbf{x})\right]_{p p}$ with its approximation $2 \int_{-\delta}^{\delta} H_{\delta}(|l|)\left(u_{\delta}\left(\mathbf{x}_{l}\right)-u_{\delta}(\mathbf{x})\right) \mathrm{d} \mathbf{x}_{l}-\kappa(\overline{\mathbf{x}}) g(\overline{\mathbf{x}})$, where $\mathrm{d} \mathbf{x}_{l}$ is the line integral along the contour $\Gamma(\mathbf{x}), \kappa(\overline{\mathbf{x}})$ is the curvature of $\partial \Omega$ at $\overline{\mathbf{x}}$, and $H_{\delta}(|r|)=\frac{c}{\delta^{3}} H\left(\frac{|r|}{\delta}\right)$ is the kernel for 1D nonlocal diffusion model. Similar to the requirements for $J$, we assume here $H:[0, \infty) \rightarrow \mathbb{R}$ to be a nonnegative and continuous function with $\int_{\mathbb{R}} H(|z|)|z|^{2} \mathrm{~d} z=1 . H(r)$ is nonincreasing in $r$, strictly positive in $[0,1]$ and vanishes for $|z|>1$. Moreover, we add a further requirement on $H$ that $\int_{\mathbb{R}} H(z) \mathrm{d} z:=C_{H}<\infty$. Here we note that $2 \int_{-\delta}^{\delta} H_{\delta}(|l|)\left(u_{\delta}\left(\mathbf{x}_{l}\right)-u_{\delta}(\mathbf{x})\right) \mathrm{d} \mathbf{x}_{l}$ is a nonlocal version of the Laplace-Beltrami operator defined on $\Gamma(\mathbf{x})$. Substituting the above two approximations into (2.2), we obtain the following model

$$
\begin{aligned}
& -2 \int_{\Omega} J_{\delta}(|\mathbf{x}-\mathbf{y}|)\left(u_{\delta}(\mathbf{y})-u_{\delta}(\mathbf{x})\right) \mathrm{d} \mathbf{y}-2 M_{\delta}(\mathbf{x}) \int_{-\delta}^{\delta} H_{\delta}(|l|)\left(u_{\delta}\left(\mathbf{x}_{l}\right)-u_{\delta}(\mathbf{x})\right) \mathrm{d} \mathbf{x}_{l} \\
& =f(\mathbf{x})-\int_{\mathbb{R}^{N} \backslash \Omega} J_{\delta}(|\mathbf{x}-\mathbf{y}|)\left[|(\mathbf{y}-\overline{\mathbf{x}}) \cdot \mathbf{n}(\overline{\mathbf{x}})|^{2}-|(\mathbf{x}-\overline{\mathbf{x}}) \cdot \mathbf{n}(\overline{\mathbf{x}})|^{2}\right] \mathrm{d} \mathbf{y} f(\mathbf{x}) \\
& \quad+\left(2 \int_{\mathbb{R}^{N} \backslash \Omega} J_{\delta}(|\mathbf{x}-\mathbf{y}|)(\mathbf{y}-\mathbf{x}) \cdot \mathbf{n}(\overline{\mathbf{x}}) \mathrm{d} \mathbf{y}-M_{\delta}(\mathbf{x}) \kappa(\overline{\mathbf{x}})\right) g(\overline{\mathbf{x}}) .
\end{aligned}
$$

where

$$
M_{\delta}(\mathbf{x}):=\int_{\mathbb{R}^{N} \backslash \Omega} J_{\delta}(|\mathbf{x}-\mathbf{y}|)\left[|(\mathbf{y}-\mathbf{x}) \cdot \mathbf{p}(\overline{\mathbf{x}})|^{2}-|(\mathbf{y}-\overline{\mathbf{x}}) \cdot \mathbf{n}(\overline{\mathbf{x}})|^{2}+|(\mathbf{x}-\overline{\mathbf{x}}) \cdot \mathbf{n}(\overline{\mathbf{x}})|^{2}\right] \mathrm{d} \mathbf{y} .
$$

Thus, by defining the nonlocal operator

$$
L_{N \delta} u:=-2 \int_{\Omega} J_{\delta}(|\mathbf{x}-\mathbf{y}|)(u(\mathbf{y})-u(\mathbf{x})) \mathrm{d} \mathbf{y}-2 M_{\delta}(\mathbf{x}) \int_{-\delta}^{\delta} H_{\delta}(|l|)\left(u\left(\mathbf{x}_{l}\right)-u(\mathbf{x})\right) \mathrm{d} \mathbf{x}_{l}
$$

and

$$
\begin{aligned}
f_{\delta}(\mathbf{x}):= & f(\mathbf{x})-\int_{\mathbb{R}^{N} \backslash \Omega} J_{\delta}(|\mathbf{x}-\mathbf{y}|)\left[|(\mathbf{y}-\overline{\mathbf{x}}) \cdot \mathbf{n}(\overline{\mathbf{x}})|^{2}-|(\mathbf{x}-\overline{\mathbf{x}}) \cdot \mathbf{n}(\overline{\mathbf{x}})|^{2}\right] \mathrm{d} \mathbf{y} f(\mathbf{x}) \\
& +\left(2 \int_{\mathbb{R}^{N} \backslash \Omega} J_{\delta}(|\mathbf{x}-\mathbf{y}|)(\mathbf{y}-\mathbf{x}) \cdot \mathbf{n}(\overline{\mathbf{x}}) \mathrm{d} \mathbf{y}-M_{\delta}(\mathbf{x}) \kappa(\overline{\mathbf{x}})\right) g(\overline{\mathbf{x}})
\end{aligned}
$$

the proposed algorithm is equivalent to the following nonlocal integral equation

$$
\left\{\begin{array}{l}
L_{N \delta} u_{\delta}=f_{\delta}, \text { in } \Omega \\
\int_{\Omega} u_{\delta} \mathrm{d} \mathbf{x}=0
\end{array}\right.
$$

The corresponding nonlocal weak formulation can then be introduced

$$
B_{\delta}\left(u_{\delta}, v\right)=\left(f_{\delta}, v\right)_{L^{2}(\Omega)},
$$

where $B_{\delta}(u, v)$ denotes a nonsymmetric bilinear form $B_{\delta}(u, v):=\left(L_{\delta} u, v\right)$. We note that

$$
\begin{aligned}
& -2 \int_{\Omega} \int_{\Omega} J_{\delta}(|\mathbf{x}-\mathbf{y}|)(u(\mathbf{y})-u(\mathbf{x})) \mathrm{d} \mathbf{y} v(\mathbf{x}) \mathrm{d} \mathbf{x} \\
& \quad=-\int_{\Omega} \int_{\Omega} J_{\delta}(|\mathbf{x}-\mathbf{y}|)(u(\mathbf{y})-u(\mathbf{x})) v(\mathbf{x}) \mathrm{d} \mathbf{y} \mathrm{d} \mathbf{x}-\int_{\Omega} \int_{\Omega} J_{\delta}(|\mathbf{y}-\mathbf{x}|)(u(\mathbf{x})-u(\mathbf{y})) v(\mathbf{y}) \mathrm{d} \mathbf{y} \mathrm{d} \mathbf{x} \\
& \quad=\int_{\Omega} \int_{\Omega} J_{\delta}(|\mathbf{x}-\mathbf{y}|)[u(\mathbf{y})-u(\mathbf{x})][v(\mathbf{y})-v(\mathbf{x})] \mathrm{d} \mathbf{y} \mathrm{d} \mathbf{x}
\end{aligned}
$$


and

$$
\begin{aligned}
-2 \int_{\Omega} & M_{\delta}(\mathbf{x}) \int_{-\delta}^{\delta} H_{\delta}(|l|)\left(u\left(\mathbf{x}_{l}\right)-u(\mathbf{x})\right) \mathrm{d} \mathbf{x}_{l} v(\mathbf{x}) \mathrm{d} \mathbf{x} \\
= & -2 \int_{\Omega} \int_{\Omega} M_{\delta}(\mathbf{x}) H_{\delta}(|l|) D(\mathbf{x}, \mathbf{y})(u(\mathbf{y})-u(\mathbf{x})) v(\mathbf{x}) \mathrm{d} \mathbf{y} \mathrm{d} \mathbf{x} \\
= & \int_{\Omega} \int_{\Omega} M_{\delta}(\mathbf{x}) H_{\delta}(|l|) D(\mathbf{x}, \mathbf{y})(u(\mathbf{y})-u(\mathbf{x}))[v(\mathbf{y})-v(\mathbf{x})] \mathrm{d} \mathbf{y} \mathrm{d} \mathbf{x} \\
& -\int_{\Omega} \int_{\Omega} M_{\delta}(\mathbf{y}) H_{\delta}(|l|) D(\mathbf{y}, \mathbf{x})(u(\mathbf{x})-u(\mathbf{y})) v(\mathbf{x}) \mathrm{d} \mathbf{x} \mathrm{d} \mathbf{y} \\
& -\int_{\Omega} \int_{\Omega} M_{\delta}(\mathbf{x}) H_{\delta}(|l|) D(\mathbf{x}, \mathbf{y})(u(\mathbf{y})-u(\mathbf{x})) v(\mathbf{x}) \mathrm{d} \mathbf{y} \mathrm{d} \mathbf{x} \\
= & \int_{\Omega} \int_{\Omega} M_{\delta}(\mathbf{x}) H_{\delta}(|l|) D(\mathbf{x}, \mathbf{y})[u(\mathbf{y})-u(\mathbf{x})][v(\mathbf{y})-v(\mathbf{x})]\left|\mathbf{r}^{\prime}(\mathbf{y})\right| \mathrm{d} \mathbf{y} \mathrm{d} \mathbf{x} \\
& +\int_{\Omega} \int_{\Omega}\left[M_{\delta}(\mathbf{y}) \frac{\left|\mathbf{r}^{\prime}(\mathbf{x})\right|}{\left|\mathbf{r}^{\prime}(\mathbf{y})\right|}-M_{\delta}(\mathbf{x})\right] H_{\delta}(|l|) D(\mathbf{x}, \mathbf{y})(u(\mathbf{y})-u(\mathbf{x})) v(\mathbf{x})\left|\mathbf{r}^{\prime}(\mathbf{y})\right| \mathrm{d} \mathbf{y} \mathrm{d} \mathbf{x} \\
= & \int_{\Omega_{\delta}} M_{\delta}(\mathbf{x}) \int_{-\delta}^{\delta} H_{\delta}(|l|)\left[u\left(\mathbf{x}_{l}\right)-u(\mathbf{x})\right]\left[v\left(\mathbf{x}_{l}\right)-v(\mathbf{x})\right] \mathrm{d} \mathbf{x}_{l} \mathrm{~d} \mathbf{x} \\
& +\int_{\Omega_{\delta}} \int_{-\delta}^{\delta}\left[M_{\delta}\left(\mathbf{x}_{l}\right) \frac{\left|\mathbf{r}^{\prime}(\mathbf{x})\right|}{\left|\mathbf{r}^{\prime}\left(\mathbf{x}_{l}\right)\right|}-M_{\delta}(\mathbf{x})\right] H_{\delta}(|l|)\left[u\left(\mathbf{x}_{l}\right)-u(\mathbf{x})\right] \mathrm{d} \mathbf{x}_{l} v(\mathbf{x}) \mathrm{d} \mathbf{x},
\end{aligned}
$$

where $\mathbf{r}$ is the bijective parametrization of $\Gamma(\mathbf{x}),\left|\mathbf{r}^{\prime}(\mathbf{x})\right|$ is the Jacobian of $\mathbf{r}$, and $D(\mathbf{x}, \mathbf{y})$ denotes a Dirac-Delta function:

$$
D(\mathbf{x}, \mathbf{y}):=\lim _{\epsilon \rightarrow 0} \epsilon^{-1} \psi(\operatorname{dist}(\mathbf{y}, \Gamma(\mathbf{x})) / \epsilon), \text { where } \psi \text { is a mollifier function on } \mathbb{R} .
$$

Therefore

$$
\begin{aligned}
B_{\delta}(u, v)= & \int_{\Omega} \int_{\Omega} J_{\delta}(|\mathbf{x}-\mathbf{y}|)[u(\mathbf{y})-u(\mathbf{x})][v(\mathbf{y})-v(\mathbf{x})] \mathrm{d} \mathbf{y} \mathrm{d} \mathbf{x} \\
& +\int_{\Omega_{\delta}} M_{\delta}(\mathbf{x}) \int_{-\delta}^{\delta} H_{\delta}(|l|)\left[u\left(\mathbf{x}_{l}\right)-u(\mathbf{x})\right]\left[v\left(\mathbf{x}_{l}\right)-v(\mathbf{x})\right] \mathrm{d} \mathbf{x}_{l} \mathrm{~d} \mathbf{x} \\
& +\int_{\Omega_{\delta}} \int_{-\delta}^{\delta}\left[M_{\delta}\left(\mathbf{x}_{l}\right) \frac{\left|\mathbf{r}^{\prime}(\mathbf{x})\right|}{\left|\mathbf{r}^{\prime}\left(\mathbf{x}_{l}\right)\right|}-M_{\delta}(\mathbf{x})\right] H_{\delta}(|l|)\left[u\left(\mathbf{x}_{l}\right)-u(\mathbf{x})\right] \mathrm{d} \mathbf{x}_{l} v(\mathbf{x}) \mathrm{d} \mathbf{x} .
\end{aligned}
$$

We then consider the nonlocal energy seminorm $\|\cdot\|_{S_{\delta}}$ as

$$
\|u\|_{S_{\delta}}^{2}=\int_{\Omega} \int_{\Omega} J_{\delta}(|\mathbf{x}-\mathbf{y}|)[u(\mathbf{y})-u(\mathbf{x})]^{2} \mathrm{~d} \mathbf{y} \mathrm{d} \mathbf{x}+\int_{\Omega_{\delta}} M_{\delta}(\mathbf{x}) \int_{-\delta}^{\delta} H_{\delta}(|l|)\left[u\left(\mathbf{x}_{l}\right)-u(\mathbf{x})\right]^{2} \mathrm{~d} \mathbf{x}_{l} \mathrm{~d} \mathbf{x},
$$

with corresponding constrained energy space given by

$$
S_{\delta}(\Omega)=\left\{u \in L^{2}(\Omega):\|u\|_{S_{\delta}}<\infty, \int_{\Omega} u \mathrm{~d} \mathbf{x}=0\right\} .
$$

Given the nonlocal Poincaré inequality which will be addressed in the next section, we will see that $\|\cdot\|_{S_{\delta}}$ is actually a full norm. Similar to [50], one can show that the constrained energy space $S_{\delta}(\Omega)$ is a Hilbert space under the given assumptions for the kernels $J$ and $H$. We note that for a fixed $\delta$ and integrable kernels $J, H$, based on the results in $[27,50]$ we have

$$
\|u\|_{S_{\delta}}^{2} \leq C_{1}(\delta)\left(\|u\|_{L^{2}(\Omega)}^{2}+\delta\|u\|_{L^{2}\left(\Omega_{\delta}\right)}^{2}\right) \leq C_{2}(\delta)\|u\|_{L^{2}(\Omega)}^{2},
$$


where $C_{1}(\delta), C_{2}(\delta)$ are constants independent of $u$ but depends on $\delta$. Together with the Poincaré inequality, we immediately obtain the equivalence result between $S_{\delta}(\Omega)$ and the space of $L^{2}(\Omega)$ functions with zero mean.

Remark 2.1. A similar form of the flux condition (2.2) has been proposed in the previous literature, e.g., $[19,61]$. By comparing the second term of (2.2) with the first case in [19], one can see that the second term of (2.2) can be obtained by taking $G=G_{1}$ in [19] and modifying the correction term $\int_{\mathbb{R}^{N} \backslash \Omega} G_{\delta}(\mathbf{x}, \mathbf{x}-\mathbf{y}) g(\mathbf{y})$ dy as $\int_{\mathbb{R}^{N} \backslash \Omega} G_{\delta}(\mathbf{x}, \mathbf{x}-\mathbf{y}) \frac{1}{2}(g(\mathbf{x})+g(\mathbf{y}))$ dy. Actually, this modification is sufficient to provide a nonlocal Neumanntype condition with second order accuracy in the 1D case, as shown in [61]. However, in higher dimensional cases we need to add the third term of (2.2) to achieve second order accuracy.

Remark 2.2. Note that in the current paper we focus on the $2 \mathrm{D}$ nonlocal diffusion problem, while the idea can be further extended to the 3D cases and to more general nonlocal IDEs, which will be addressed in future work.

\section{WELL-POSEDNESS AND ASYMPTOTIC PROPERTY}

In this section, we first address the well-posedness of the proposed nonlocal Neumann volume-constrained problem by providing a nonlocal Poincaré-type inequality based on the estimates for boundary curvature $\kappa(\mathbf{x})$ and its derivative $\kappa^{\prime}(\mathbf{x})$. The coercivity and boundedness of the nonsymmetric bilinear operator $B_{\delta}(\cdot, \cdot)$ defined in (2.7) follow, which yield the well-posedness of the variational problem. Furthermore, we study the consistency of the nonlocal problem with the classical local model. Specifically, following the framework introduced in [63] we prove the uniform embedding property and the precompact property of the proposed norm $S_{\delta}$, and then show the asymptotic property of the solution of $(2.5)$ as $\delta \rightarrow 0$, i.e., the solution $u_{\delta}$ converges to the solution $u_{0}$ from the limiting local model (2.1). Here for simplicity we consider the case when $g(\mathbf{x})=0$, and defer discussion of inhomogeneous boundary conditions until Remark 3.11. For the limiting local model one can define the corresponding inner product $\|u\|_{S_{0}}=\|\nabla u\|_{L^{2}(\Omega)}$, the bilinear form $B_{0}(u, v)=(\nabla u, \nabla v)$ and the constrained energy space $S_{0}=\left\{u \in H^{1}(\Omega): \int_{\Omega} u \mathrm{~d} \mathbf{x}=0\right\}$. Throughout this section, we consider the symbol " $C$ " to indicate a generic constant that is independent of $\delta$, but may have different numerical values in different situations. Moreover, we introduce the following notation for simplicity:

$$
\begin{aligned}
b_{\delta}(u, v) & :=\int_{\Omega} \int_{\Omega} J_{\delta}(|\mathbf{x}-\mathbf{y}|)(u(\mathbf{y})-u(\mathbf{x}))(v(\mathbf{y})-v(\mathbf{x})) \mathrm{d} \mathbf{y} \mathrm{d} \mathbf{x}, \\
h_{\delta}(u, v) & :=\int_{\Omega_{\delta}} M_{\delta}(\mathbf{x}) \int_{-\delta}^{\delta} H_{\delta}(|l|)\left[u\left(\mathbf{x}_{l}\right)-u(\mathbf{x})\right]\left[v\left(\mathbf{x}_{l}\right)-v(\mathbf{x})\right] \mathrm{d} \mathbf{x}_{l} \mathrm{~d} \mathbf{x}, \\
I_{\delta}(\mathbf{x}, \mathbf{y}) & :=|(\mathbf{y}-\mathbf{x}) \cdot \mathbf{p}(\overline{\mathbf{x}})|^{2}-|(\mathbf{y}-\overline{\mathbf{x}}) \cdot \mathbf{n}(\overline{\mathbf{x}})|^{2}+|(\mathbf{x}-\overline{\mathbf{x}}) \cdot \mathbf{n}(\overline{\mathbf{x}})|^{2} .
\end{aligned}
$$

We first have the following estimates of the function $M_{\delta}(\mathbf{x})$ for each $\mathbf{x} \in \Omega_{\delta}$ :

Lemma 3.1. For $l \in[-\delta, \delta]$, and assuming that there exist constants $d, D>0$ such that $\left|\kappa^{\prime}(\mathbf{z})\right| \leq D,|\kappa(\mathbf{z})| \leq D$ and $\sup _{|\xi| \leq d}\left|\frac{\kappa^{\prime}\left(\mathbf{z}_{\xi}\right)}{\kappa(\mathbf{z})}\right| \leq D$ for almost every $\mathbf{z} \in \partial \Omega$, there exists $a 0<\bar{\delta} \leq d$ such that for $\delta \leq \bar{\delta}$ for almost every $\mathbf{x} \in \partial \Omega$ we have $0 \leq M_{\delta}(\mathbf{x}) \leq C$ and

$$
\begin{aligned}
\left|M_{\delta}(\mathbf{x})-M_{\delta}\left(\mathbf{x}_{l}\right) \frac{\left|\mathbf{r}^{\prime}(\mathbf{x})\right|}{\left|\mathbf{r}^{\prime}\left(\mathbf{x}_{l}\right)\right|}\right| & \leq C_{M} \delta^{2} \\
\left|\frac{M_{\delta}(\mathbf{x})\left|\mathbf{r}^{\prime}\left(\mathbf{x}_{l}\right)\right|-M_{\delta}\left(\mathbf{x}_{l}\right)\left|\mathbf{r}^{\prime}(\mathbf{x})\right|}{M_{\delta}(\mathbf{x})\left|\mathbf{r}^{\prime}\left(\mathbf{x}_{l}\right)\right|}\right| & \leq C_{N} \delta
\end{aligned}
$$

where $C_{M}, C_{N}$ are constants independent of $\delta$. 

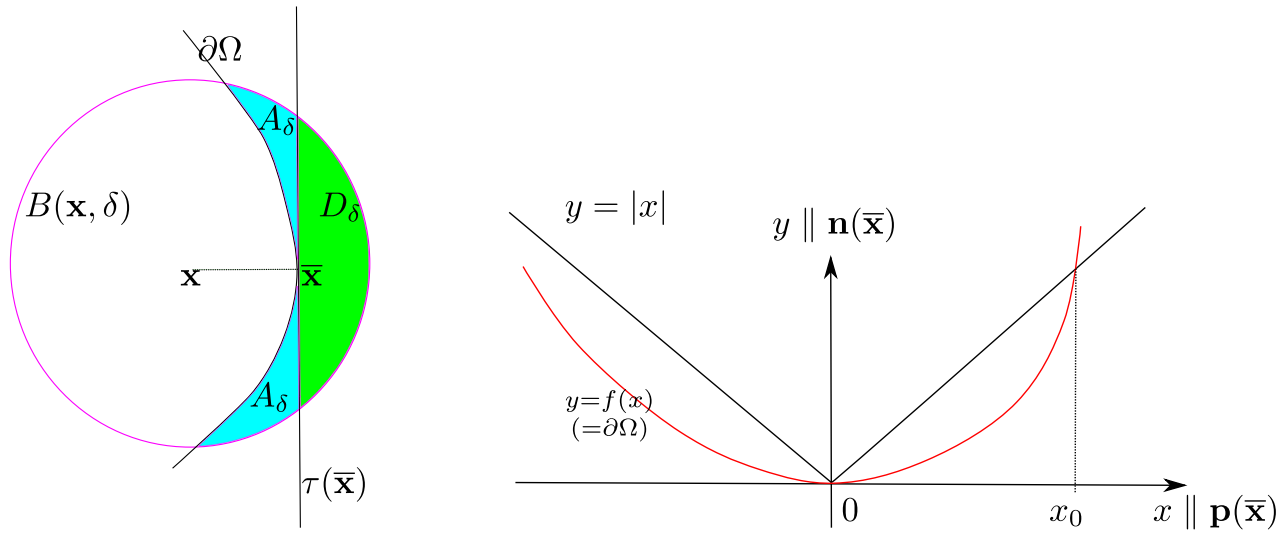

FIGURE 2. Notation for the geometric estimates in Lemma 3.1. Left: illustration of regions $D_{\delta}$ and $A_{\delta}$. Green represents $D_{\delta}$, the region in $B(\mathbf{x}, \delta)$ which lies on the other side of the tangential line at $\overline{\mathbf{x}}$ with respect to $\Omega$. Cyan represents $A_{\delta}$, the region in $B(\mathbf{x}, \delta)$ which lies between $\partial \Omega$ and the tangential line. Right: representation of the Cartesian coordinate system locally near $\overline{\mathbf{x}}$. Here the region $A_{\delta}$ lies below the red curve $y=f(x)$.

We show now that $0 \leq M_{\delta}(\mathbf{x}) \leq C$. Note that

$$
M_{\delta}(\mathbf{x})=\int_{\left(\mathbb{R}^{N} \backslash \Omega\right)_{\delta}} J_{\delta}(|\mathbf{x}-\mathbf{y}|) I_{\delta} \mathrm{d} \mathbf{y}=\int_{D_{\delta}} J_{\delta}(|\mathbf{x}-\mathbf{y}|) I_{\delta} \mathrm{d} \mathbf{y}+\int_{A_{\delta}} J_{\delta}(|\mathbf{x}-\mathbf{y}|) I_{\delta} \mathrm{d} \mathbf{y} .
$$

With $\tau(\overline{\mathbf{x}})$ representing the tangent line to $\partial \Omega$ at $\overline{\mathbf{x}}$, here $D_{\delta}$ is the region of $B(\mathbf{x}, \delta)$ on the side of $\tau(\overline{\mathbf{x}})$ not containing $\Omega$ (as shown in the green region in the left plot of Fig. 2), and $A_{\delta}:=B(\mathbf{x}, \delta) \backslash\left(D_{\delta} \cup \Omega\right.$ ) (as shown in the cyan region in the left plot of Fig. 2). We consider first the $D_{\delta}$ part. One can rewrite $\mathbf{y} \in D_{\delta}$ as $\mathbf{x}+(r \cos (\theta), r \sin (\theta))$ with $s_{x}<r<\delta$ and $-\pi / 2<-\arccos \left(s_{x} / r\right) \leq \theta \leq \arccos \left(s_{x} / r\right) \leq \pi / 2$, which yields

$$
\begin{aligned}
\int_{D_{\delta}} J_{\delta}(|\mathbf{x}-\mathbf{y}|) I_{\delta} \mathrm{d} \mathbf{y} & =\int_{s_{x}}^{\delta} J_{\delta}(r) \int_{-\arccos \left(s_{x} / r\right)}^{\arccos \left(s_{x} / r\right)} I_{\delta}(\mathbf{x}, \mathbf{y}) r \mathrm{~d} \theta \mathrm{d} r \\
& =2 \int_{s_{x}}^{\delta} J_{\delta}(r) r^{2} \sqrt{1-\left(s_{x} / r\right)^{2}} s_{x} \mathrm{~d} r .
\end{aligned}
$$

From (3.3) we can see that $\int_{D_{\delta}} J_{\delta}(|\mathbf{x}-\mathbf{y}|) I_{\delta} \mathrm{d} \mathbf{y} \geq 0$ and

$$
M_{\delta}(\mathbf{x}) \geq \int_{A_{\delta}} J_{\delta}(|\mathbf{x}-\mathbf{y}|)\left[|(\mathbf{y}-\overline{\mathbf{x}}) \cdot \mathbf{p}(\overline{\mathbf{x}})|^{2}-|(\mathbf{y}-\overline{\mathbf{x}}) \cdot \mathbf{n}(\overline{\mathbf{x}})|^{2}\right] \mathrm{d} \mathbf{y} .
$$

Therefore it suffices to show now that

$$
|(\mathbf{y}-\overline{\mathbf{x}}) \cdot \mathbf{p}(\overline{\mathbf{x}})| \geq|(\mathbf{y}-\overline{\mathbf{x}}) \cdot \mathbf{n}(\overline{\mathbf{x}})| \quad \forall \mathbf{y} \in A_{\delta} .
$$

We adopt a Cartesian coordinate system as shown in the right plot of Figure 2, assuming that $\overline{\mathbf{x}}$ coincides with the origin, $\mathbf{p}(\overline{\mathbf{x}})$ is oriented along the positive direction of the $x$-axis while $\mathbf{n}(\overline{\mathbf{x}})$ coincides with the negative direction of the $y$-axis. We then have $\overline{\mathbf{x}}=(0,0), \tau(\overline{\mathbf{x}})=\{y=0\}, \Omega \subseteq\{y \geq 0\}$, and let $y=f(x)$ be the curve describing $\partial \Omega$. We note that any point $\mathbf{y}$ lying below $y=|x|$ satisfies (3.5). Assuming that there exists a point $\mathbf{z} \in \partial \Omega$ lying above $y=|x|$, there exists $x_{0} \neq 0$ such that $f\left(x_{0}\right)=\left|x_{0}\right|$ and $\left(x_{0}, f\left(x_{0}\right)\right) \in \partial \Omega$. For simplicity we consider the case where $x_{0}>0$ since the case where $x_{0}<0$ is analogous. Since $f^{\prime}(0)=0$, by continuity there 
exists at least one point $x_{1} \in\left(0, x_{0}\right)$ such that $f^{\prime}\left(x_{1}\right) \geq 1$. Let $x_{2}:=\inf \left\{t>0: f^{\prime}(t) \geq 1\right\} \leq x_{1} \leq x_{0}$, then by the regularity of $f$ we have $x_{2}>0$. Thus $f^{\prime}\left(x_{2}\right)-f^{\prime}(0)=1=\int_{0}^{x_{2}} f^{\prime \prime}(s) \mathrm{d} s$. Moreover, the unsigned curvature of the graph of $f$ can be given by $\left|f^{\prime \prime}(x)\right|\left(1+f^{\prime}(x)^{2}\right)^{-3 / 2}$. Due to the finiteness of the curvature of $\partial \Omega$, and the fact that $f^{\prime}(x)^{2} \leq 1$ for all $x \in\left[0, x_{2}\right]$, we obtain $D \geq\left|f^{\prime \prime}(x)\right|\left(1+f^{\prime}(x)^{2}\right)^{-3 / 2}$ and therefore

$$
\left|f^{\prime \prime}(x)\right| \leq D\left(1+f^{\prime}(x)^{2}\right)^{3 / 2} \leq 2 \sqrt{2} D, \quad \forall x \in\left[0, x_{2}\right] .
$$

Hence

$$
1=\int_{0}^{x_{2}} f^{\prime \prime}(s) \mathrm{d} s \leq \int_{0}^{x_{2}}\left|f^{\prime \prime}(s)\right| \mathrm{d} s \leq 2 \sqrt{2} x_{2} D \Rightarrow x_{2} \geq \frac{1}{2 \sqrt{2} D} .
$$

But, since $x_{2} \leq x_{0}$, this means that the first intersection point between $y=f(x)$ and $y=|x|$ (which we denote as $\left.\mathbf{w}=\left(x_{0},\left|x_{0}\right|\right)\right)$ has distance at least $x_{2}$ from $\overline{\mathbf{x}}=(0,0)$. Thus, for sufficiently small $\delta \leq \frac{1}{4 \sqrt{2} D}=: \delta_{1}$, we get

$$
|\mathbf{w}-\overline{\mathbf{x}}| \geq x_{2} \geq \frac{1}{2 \sqrt{2} D} \geq 2 \delta=\sup _{p, q \in B(\mathbf{x}, \delta)}|p-q| .
$$

Therefore, $\mathbf{w} \notin B(\mathbf{x}, \delta)$, and the entire region $A_{\delta}$ lies below $y=|x|$. Consequently, any $\mathbf{y} \in A_{\delta}$ satisfies $|(\mathbf{y}-\overline{\mathbf{x}}) \cdot \mathbf{p}(\overline{\mathbf{x}})| \geq|(\mathbf{y}-\overline{\mathbf{x}}) \cdot \mathbf{n}(\overline{\mathbf{x}})|$ and in turn $M_{\delta} \geq 0$. On the other hand, with the $C^{3}$ regularity of $\Omega$ and by Taylor expansion $B(\mathbf{x}, \delta) \cap \partial \Omega$ is the graph of a function of the form $y=f(x)=\frac{\kappa(\overline{\mathbf{x}})}{2} x^{2}+O\left(x^{3}\right)$. Therefore, the area $\left|A_{\delta}\right| \leq C|\kappa(\overline{\mathbf{x}})|\left(\delta^{2}-s_{x}^{2}\right)^{3 / 2} \leq C D \delta^{3}$. Hence

$$
\begin{aligned}
M_{\delta}(\mathbf{x}) & \leq\left|\int_{D_{\delta}} J_{\delta}(|\mathbf{x}-\mathbf{y}|) I_{\delta} \mathrm{d} \mathbf{y}\right|+\left|\int_{A_{\delta}} J_{\delta}(|\mathbf{x}-\mathbf{y}|) I_{\delta} \mathrm{d} \mathbf{y}\right| \\
& \leq C \sup _{r} J(|r|)\left(\delta^{-4} s_{x}\left(\delta^{2}-s_{x}^{2}\right)^{3 / 2}+D \delta\right) \leq C .
\end{aligned}
$$

To show (3.1), denoting by $D_{\delta, \mathbf{x}_{l}}, A_{\delta, \mathbf{x}_{l}}$ the analogous sets of $D_{\delta}, A_{\delta}$ at $\mathbf{x}_{l}$ instead of $\mathbf{x}$, we then have

$$
\begin{aligned}
M_{\delta}(\mathbf{x})-M_{\delta}\left(\mathbf{x}_{l}\right) \frac{\left|\mathbf{r}^{\prime}(\mathbf{x})\right|}{\left|\mathbf{r}^{\prime}\left(\mathbf{x}_{l}\right)\right|}= & \int_{D_{\delta}} J_{\delta}(|\mathbf{x}-\mathbf{y}|) I_{\delta}(\mathbf{x}, \mathbf{y}) \mathrm{d} \mathbf{y}-\frac{\left|\mathbf{r}^{\prime}(\mathbf{x})\right|}{\left|\mathbf{r}^{\prime}\left(\mathbf{x}_{l}\right)\right|} \int_{D_{\delta, \mathbf{x}_{l}}} J_{\delta}\left(\left|\mathbf{x}_{l}-\mathbf{y}\right|\right) I_{\delta}\left(\mathbf{x}_{l}, \mathbf{y}\right) \mathrm{d} \mathbf{y} \\
& +\int_{A_{\delta}} J_{\delta}(|\mathbf{x}-\mathbf{y}|) I_{\delta}(\mathbf{x}, \mathbf{y}) \mathrm{d} \mathbf{y}-\frac{\left|\mathbf{r}^{\prime}(\mathbf{x})\right|}{\left|\mathbf{r}^{\prime}\left(\mathbf{x}_{l}\right)\right|} \int_{A_{\delta, \mathbf{x}_{l}}} J_{\delta}\left(\left|\mathbf{x}_{l}-\mathbf{y}\right|\right) I_{\delta}\left(\mathbf{x}_{l}, \mathbf{y}\right) \mathrm{d} \mathbf{y} .
\end{aligned}
$$

With the definition of $\mathbf{x}_{l}$ and the regularity assumptions on $\Omega$, it holds $s_{\mathbf{x}_{l}}:=\operatorname{dist}\left(\mathbf{x}_{l}, \partial \Omega\right)=\operatorname{dist}(\mathbf{x}, \partial \Omega)$. We obtain

$$
\int_{D_{\delta, \mathbf{x}_{l}}} J_{\delta}\left(\left|\mathbf{x}_{l}-\mathbf{y}\right|\right) I_{\delta}\left(\mathbf{x}_{l}, \mathbf{y}\right) \mathrm{d} \mathbf{y}=\int_{D_{\delta}} J_{\delta}(|\mathbf{x}-\mathbf{y}|) I_{\delta}(\mathbf{x}, \mathbf{y}) \mathrm{d} \mathbf{y} .
$$

Moreover, with the coordinate system as shown in the right plot of Figure 2, we have $\left|\mathbf{r}^{\prime}(\mathbf{x})\right|=1$ and $\left|\mathbf{r}^{\prime}\left(\mathbf{x}_{l}\right)\right|=$ $\sqrt{1+\left(f^{\prime}(x)\right)^{2}}$. Since for any point $\mathbf{x}_{l}=\left(x_{l}, f\left(x_{l}\right)\right)$ in $B(\mathbf{x}, \delta),\left|f^{\prime}\left(x_{l}\right)\right|=\left|x_{l} f^{\prime \prime}(\xi)\right| \leq C \delta$ for some $\xi \in\left[0, x_{l}\right]$, therefore

$$
\left|\frac{\left|\mathbf{r}^{\prime}(\mathbf{x})\right|}{\left|\mathbf{r}^{\prime}\left(\mathbf{x}_{l}\right)\right|}-1\right|=\left|\frac{1}{\sqrt{1+\left(f^{\prime}\left(x_{l}\right)\right)^{2}}}-1\right|=\frac{1}{2}\left(f^{\prime}\left(x_{l}\right)\right)^{2}+O\left(\delta^{4}\right) \leq C \delta^{2}
$$

and hence together with (3.3) we obtain

$$
\int_{D_{\delta}} J_{\delta}(|\mathbf{x}-\mathbf{y}|) I_{\delta}(\mathbf{x}, \mathbf{y}) \mathrm{d} \mathbf{y}-\frac{\left|\mathbf{r}^{\prime}(\mathbf{x})\right|}{\left|\mathbf{r}^{\prime}\left(\mathbf{x}_{l}\right)\right|} \int_{D_{\delta, \mathbf{x}_{l}}} J_{\delta}\left(\left|\mathbf{x}_{l}-\mathbf{y}\right|\right) I_{\delta}\left(\mathbf{x}_{l}, \mathbf{y}\right) \mathrm{d} \mathbf{y} \leq C \delta^{2} .
$$

To estimate $\int_{A_{\delta}} J_{\delta}(|\mathbf{x}-\mathbf{y}|) I_{\delta}(\mathbf{x}, \mathbf{y}) \mathrm{d} \mathbf{y}-\int_{A_{\delta, \mathbf{x}_{l}}} J_{\delta}\left(\left|\mathbf{x}_{l}-\mathbf{y}\right|\right) I_{\delta}\left(\mathbf{x}_{l}, \mathbf{y}\right) \mathrm{d} \mathbf{y}$, let $R$ be the rototranslation mapping such that

$$
R\left(\overline{\mathbf{x}}_{l}\right)=\overline{\mathbf{x}}, \quad R\left(\mathbf{p}\left(\overline{\mathbf{x}}_{l}\right)\right)=\mathbf{p}(\overline{\mathbf{x}}), \quad R\left(\mathbf{n}\left(\overline{\mathbf{x}}_{l}\right)\right)=\mathbf{n}(\overline{\mathbf{x}}) .
$$


With such construction we note that the curves $R\left(B\left(\mathbf{x}_{l}, \delta\right) \cap \partial \Omega\right)$ and $B(\mathbf{x}, \delta) \cap \partial \Omega$ share the same tangential lines at $\overline{\mathbf{x}}$. Meanwhile, $B(\mathbf{x}, \delta) \cap \partial \Omega$ and $R\left(B\left(\mathbf{x}_{l}, \delta\right) \cap \partial \Omega\right)$ have different curvatures $\kappa(\overline{\mathbf{x}})$ and $\kappa\left(\overline{\mathbf{x}}_{l}\right)$, respectively. When $\delta \ll 1 / D$, we have the arc lengths of $R\left(B\left(\mathbf{x}_{l}, \delta\right) \cap \partial \Omega\right)$ and $B(\mathbf{x}, \delta) \cap \partial \Omega$ satisfying $\left|R\left(B\left(\mathbf{x}_{l}, \delta\right) \cap \partial \Omega\right)\right| \leq$ $2 \sqrt{\delta^{2}-s_{x}^{2}}+C \kappa\left(\overline{\mathbf{x}}_{l}\right)\left(\delta^{2}-s_{x}^{2}\right)$ and $|B(\mathbf{x}, \delta) \cap \partial \Omega| \leq 2 \sqrt{\delta^{2}-s_{x}^{2}}+C \kappa(\overline{\mathbf{x}})\left(\delta^{2}-s_{x}^{2}\right)$. Moreover, the spread $d_{\mathcal{H}}(B(\mathbf{x}, \delta) \cap$ $\left.\partial \Omega, R\left(B\left(\mathbf{x}_{l}, \delta\right) \cap \partial \Omega\right)\right)$ is bounded by

$$
\begin{aligned}
& d_{\mathcal{H}}\left(B(\mathbf{x}, \delta) \cap \partial \Omega, R\left(B\left(\mathbf{x}_{l}, \delta\right) \cap \partial \Omega\right)\right) \\
& \quad=\max \left\{\sup _{z \in B(\mathbf{x}, \delta) \cap \partial \Omega} \operatorname{dist}\left(z, R\left(B\left(\mathbf{x}_{l}, \delta\right) \cap \partial \Omega\right)\right), \sup _{z \in R\left(B\left(\mathbf{x}_{l}, \delta\right) \cap \partial \Omega\right)} \operatorname{dist}(z, B(\mathbf{x}, \delta) \cap \partial \Omega)\right\} \\
& \quad \leq C\left|\kappa\left(\overline{\mathbf{x}}_{l}\right)-\kappa(\overline{\mathbf{x}})\right|\left(\delta^{2}-s_{x}^{2}\right) .
\end{aligned}
$$

Therefore, noting that the quantities $J_{\delta}, I_{\delta}$ and $\mathrm{d} \mathbf{y}$ are invariant under $R$, and $\left|I_{\delta}\left(\mathbf{x}_{l}, \mathbf{y}\right)\right|,\left|I_{\delta}(\mathbf{x}, \mathbf{y})\right| \leq 3 \delta^{2}$, one has

$$
\begin{aligned}
& \left|\int_{A_{\delta, \mathbf{x}_{l}}} J_{\delta}\left(\left|\mathbf{x}_{l}-\mathbf{y}\right|\right) I_{\delta}\left(\mathbf{x}_{l}, \mathbf{y}\right) \mathrm{d} \mathbf{y}-\int_{A_{\delta}} J_{\delta}(|\mathbf{x}-\mathbf{y}|) I_{\delta}(\mathbf{x}, \mathbf{y}) \mathrm{d} \mathbf{y}\right| \\
& \quad=\left|\int_{R\left(A_{\left.\delta, \mathbf{x}_{l}\right)}\right.} J_{\delta}(|\mathbf{x}-\mathbf{y}|) I_{\delta}(\mathbf{x}, \mathbf{y}) \mathrm{d} \mathbf{y}-\int_{A_{\delta}} J_{\delta}(|\mathbf{x}-\mathbf{y}|) I_{\delta}(\mathbf{x}, \mathbf{y}) \mathrm{d} \mathbf{y}\right| \\
& \quad \leq C \int_{A_{\delta} \Delta R\left(A_{\left.\delta, \mathbf{x}_{l}\right)} J_{\delta}(|\mathbf{x}-\mathbf{y}|) \delta^{2} \mathrm{~d} \mathbf{y} \leq C\left|\kappa\left(\overline{\mathbf{x}}_{l}\right)-\kappa(\overline{\mathbf{x}})\right| \delta^{-2}\left(\delta^{2}-s_{x}^{2}\right)^{3 / 2}\right.} \leq C \sup _{|\xi| \leq|l|}\left|\kappa^{\prime}\left(\overline{\mathbf{x}}_{\xi}\right)\right| \delta^{-1}\left(\delta^{2}-s_{x}^{2}\right)^{3 / 2} \leq C \delta^{-1}\left(\delta^{2}-s_{x}^{2}\right)^{3 / 2},
\end{aligned}
$$

where the constant $C$ depends on $\sup _{r} J(|r|)$ and is independent of $\delta$. Moreover, with (3.6) and $\left|\int_{A_{\delta}} J_{\delta}(|\mathbf{x}-\mathbf{y}|) I_{\delta} \mathrm{d} \mathbf{y}\right| \leq C \sup _{r} J(|r|) \delta$ we have

$$
\left|\left(1-\frac{\left|\mathbf{r}^{\prime}(\mathbf{x})\right|}{\left|\mathbf{r}^{\prime}\left(\mathbf{x}_{l}\right)\right|}\right) \int_{A_{\delta, \mathbf{x}_{l}}} J_{\delta}\left(\left|\mathbf{x}_{l}-\mathbf{y}\right|\right) I_{\delta}\left(\mathbf{x}_{l}, \mathbf{y}\right) \mathrm{d} \mathbf{y}\right| \leq C \delta^{3} .
$$

Thus, we obtain the bound in (3.1).

We now work on the proof of (3.2) by combining (3.7) and establishing a lower bound for $M_{\delta}$. We firstly prove that

$$
\left|\frac{M_{\delta}(\mathbf{x})-M_{\delta}\left(\mathbf{x}_{l}\right)}{M_{\delta}(\mathbf{x})}\right| \leq C \delta .
$$

With the previous calculation, we have

$$
\begin{aligned}
\int_{D_{\delta}} J_{\delta}(\mathbf{x}-\mathbf{y}) I_{\delta} \mathrm{d} \mathbf{y} & =2 \int_{s_{x}}^{\delta} J_{\delta}(r) r^{2} \sqrt{1-\left(s_{x} / r\right)^{2}} s_{x} \mathrm{~d} r \geq C \delta^{-4} s_{x}\left(\delta^{2}-s_{x}^{2}\right)^{3 / 2} \\
& \geq \frac{C}{D}|\kappa(\overline{\mathbf{x}})| \delta^{-4} s_{x}\left(\delta^{2}-s_{x}^{2}\right)^{3 / 2}=C|\kappa(\overline{\mathbf{x}})| \delta^{-4} s_{x}\left(\delta^{2}-s_{x}^{2}\right)^{3 / 2}
\end{aligned}
$$

and $\int_{A_{\delta}} J_{\delta}(\mathbf{x}-\mathbf{y}) I_{\delta} \mathrm{d} \mathbf{y} \geq 0$. When $s_{x}^{2} \geq \delta^{2} / 2$ one has

$$
\left|\frac{M_{\delta}(\mathbf{x})-M_{\delta}\left(\mathbf{x}_{l}\right)}{M_{\delta}(\mathbf{x})}\right| \leq C \frac{\left|\kappa\left(\overline{\mathbf{x}}_{l}\right)-\kappa(\overline{\mathbf{x}})\right| \delta^{-2}\left(\delta^{2}-s_{x}^{2}\right)^{3 / 2}}{|\kappa(\overline{\mathbf{x}})| \delta^{-4} s_{x}\left(\delta^{2}-s_{x}^{2}\right)^{3 / 2}}=C \frac{\delta^{3}}{s_{x}} \sup _{|\xi| \leq d}\left|\frac{\kappa^{\prime}\left(\overline{\mathbf{x}}_{\xi}\right)}{\kappa(\overline{\mathbf{x}})}\right| \leq C \delta \sup _{|\xi| \leq d}\left|\frac{\kappa^{\prime}\left(\overline{\mathbf{x}}_{\xi}\right)}{\kappa(\overline{\mathbf{x}})}\right|
$$

and therefore (3.8) holds true. For $s_{x}^{2}<\delta^{2} / 2$, we just need to bound $\int_{A_{\delta}} J_{\delta}(\mathbf{x}-\mathbf{y}) I_{\delta} \mathrm{d} \mathbf{y}$ from below. For notational simplicity, we assume here the Cartesian coordinate system shown in the right plot of Figure 2 . The 
following properties hold:

$$
\begin{aligned}
& (\mathbf{y}-\overline{\mathbf{x}}) \cdot \mathbf{p}(\overline{\mathbf{x}})=x \text { coordinate of } \mathbf{y} \\
& (\mathbf{y}-\overline{\mathbf{x}}) \cdot \mathbf{n}(\overline{\mathbf{x}})=-(y \text { coordinate of } \mathbf{y})
\end{aligned}
$$

We first assume that $|\kappa(\overline{\mathbf{x}})|>0$. By Taylor approximation, $B(\mathbf{x}, \delta) \cap \partial \Omega$ is the graph of a function of the form $y=f(x)=\frac{\kappa(\overline{\mathbf{x}})}{2} x^{2}+O\left(x^{3}\right)$. Integrating it yields that the area $\left|A_{\delta}\right|=C|\kappa(\overline{\mathbf{x}})|\left(\delta^{2}-s_{x}^{2}\right)^{3 / 2}=C|\kappa(\overline{\mathbf{x}})| \delta^{3}$. Let $h \in(0,1)$ be a point where the area of $A_{\delta} \cap\{x \geq h \delta\}$ is $C|\kappa(\overline{\mathbf{x}})| \delta^{3} / 2$. With the convexity assumption of $\partial \Omega$, one has $h>1 / 2$. When $\delta \leq \delta_{1}<\frac{1}{2 D}$, the slope of $f$ (i.e., the slope of the tangent derivative of $B(\mathbf{x}, \delta) \cap \partial \Omega$ ) can reach at most $\delta D<1 / 2$. Thus the graph of $f$ lies below the line $y=x / 2$ and (3.10) gives

$$
\begin{aligned}
I_{\delta} & \geq|(\mathbf{y}-\mathbf{x}) \cdot \mathbf{p}(\overline{\mathbf{x}})|^{2}-|(\mathbf{y}-\overline{\mathbf{x}}) \cdot \mathbf{n}(\overline{\mathbf{x}})|^{2} \geq|(\mathbf{y}-\overline{\mathbf{x}}) \cdot \mathbf{p}(\overline{\mathbf{x}})|^{2}-|(\mathbf{y}-\overline{\mathbf{x}}) \cdot \mathbf{n}(\overline{\mathbf{x}})|^{2} \\
& \geq \frac{3}{4}|(\mathbf{y}-\overline{\mathbf{x}}) \cdot \mathbf{p}(\overline{\mathbf{x}})|^{2} \geq \frac{3}{4} h^{2} \delta^{2} \geq \frac{3}{16} \delta^{2}
\end{aligned}
$$

for all $\mathbf{y} \in A_{\delta} \cap\{x \geq h \delta\}$. Recalling that $J(r)$ is strictly positive for $0 \leq r \leq 1$ and therefore $\min _{r \leq 1} J(|r|)>0$, we infer

$$
\begin{aligned}
M_{\delta} & \geq \int_{A_{\delta} \cap\{x \geq h\}} \min _{r \leq 1} J(|r|) \delta^{-4}\left(|(\mathbf{y}-\overline{\mathbf{x}}) \cdot \mathbf{p}(\overline{\mathbf{x}})|^{2}-|(\mathbf{y}-\overline{\mathbf{x}}) \cdot \mathbf{n}(\overline{\mathbf{x}})|^{2}\right) \mathrm{d} \mathbf{y} \\
& \geq \frac{3 C}{32}|\kappa(\overline{\mathbf{x}})| \delta \geq C|\kappa(\overline{\mathbf{x}})| \delta .
\end{aligned}
$$

Combining with (3.7), we thus obtain the bound (3.8).

For $|\kappa(\overline{\mathbf{x}})|=0$, with domain $C^{3}$ regularity assumption and $\sup _{|\xi| \leq d}\left|\frac{\kappa^{\prime}\left(\overline{\mathbf{x}}_{\xi}\right)}{\kappa(\overline{\mathbf{x}})}\right| \leq D$ a.e., we have $\kappa\left(\overline{\mathbf{x}}_{\xi}\right) \equiv 0$ for $|\xi| \leq d$ almost everywhere and therefore $M_{\delta}\left(\overline{\mathbf{x}}_{l}\right)=M_{\delta}(\overline{\mathbf{x}})$ for $|l| \leq \delta$ and $\delta \leq d / 2$. (3.8) can then be trivially proved.

We can now prove $(3.2)$ :

$$
\left|\frac{M_{\delta}(\mathbf{x})\left|\mathbf{r}^{\prime}\left(\mathbf{x}_{l}\right)\right|-M_{\delta}\left(\mathbf{x}_{l}\right)\left|\mathbf{r}^{\prime}(\mathbf{x})\right|}{M_{\delta}(\mathbf{x})\left|\mathbf{r}^{\prime}\left(\mathbf{x}_{l}\right)\right|}\right| \leq \frac{\left|\mathbf{r}^{\prime}(\mathbf{x})\right|}{\left|\mathbf{r}^{\prime}\left(\mathbf{x}_{l}\right)\right|}\left|\frac{M_{\delta}(\mathbf{x})-M_{\delta}\left(\mathbf{x}_{l}\right)}{M_{\delta}(\mathbf{x})}\right|+\left|\frac{\left|\mathbf{r}^{\prime}(\mathbf{x})\right|}{\left|\mathbf{r}^{\prime}\left(\mathbf{x}_{l}\right)\right|}-1\right| \leq C \delta
$$

Remark 3.2. Note that in the previous proof we assumed $J(r)$ is strictly positive in $[0,1]$ such that $J(|r|) \geq$ $C_{1}>0$. However, the proof can be extended for a more general positive $J$ whose support is the entire ball $B(0,1)$. It suffices to note:

- It easily follows from the previous proof that the set

$$
\tilde{A}_{\delta}:=\left\{\mathbf{z} \in A_{\delta}:|\mathbf{z}-\mathbf{x}| \in[\delta / 3, \delta / 2]\right\}
$$

has area $C\left|A_{\delta}\right|$ for some constant $C \in(0,1)$, and on $\tilde{A}_{\delta}$ it holds $I_{\delta} \geq C_{1} \delta^{2}$, again for some constant $C_{1} \in(0,1)$.

- Since $J(r)$ is nonincreasing on $r$ and its support is the entire ball $B(0,1)$ there exists another constant $C_{2}>0$ such that $J(r) \geq C_{2}$ for $r \in[1 / 3,1 / 2]$.

Combining the above two facts, we obtain

$$
M_{\delta} \geq \int_{A_{\delta}} J_{\delta}(|\mathbf{x}-\mathbf{y}|) I_{\delta} \mathrm{d} \mathbf{y} \geq \int_{\tilde{A}_{\delta}} \min _{\delta \leq r \leq \delta / 2} J_{\delta}(r) I_{\delta} \mathrm{d} \mathbf{y} \geq C|\kappa(\overline{\mathbf{x}})| \delta .
$$


Remark 3.3. When $u \in C^{\infty}(\Omega)$, the above bounds of $M_{\delta}(\mathbf{x})$ yield

$$
\begin{aligned}
0 \leq h_{\delta}(u, u) & =\int_{\Omega_{\delta}} M_{\delta}(\mathbf{x}) \int_{-\delta}^{\delta} H_{\delta}(|l|)\left[u\left(\mathbf{x}_{l}\right)-u(\mathbf{x})\right]^{2} \mathrm{~d} \mathbf{x}_{l} \mathrm{~d} \mathbf{x} \\
& \leq \int_{\Omega_{\delta}} M_{\delta}(\mathbf{x}) \int_{-\delta}^{\delta} H_{\delta}(|l|)\left[\left|\frac{\partial u(\mathbf{x})}{\partial \mathbf{p}}\right|^{2}|l|^{2}+C|l|^{3}\right] \mathrm{d} \mathbf{x}_{l} \mathrm{~d} \mathbf{x} \\
& \leq \int_{\Omega_{\delta}} M_{\delta}(\mathbf{x})\left|\frac{\partial u(\mathbf{x})}{\partial \mathbf{p}}\right|^{2} \mathrm{~d} \mathbf{x}+C\left|\Omega_{\delta}\right| \delta \leq C\left|\Omega_{\delta}\right|\left(\left|\frac{\partial u(\mathbf{x})}{\partial \mathbf{p}}\right|^{2}+\delta\right) .
\end{aligned}
$$

Combining with the results in [63], we have $\lim _{\delta \rightarrow 0}\|u\|_{S_{\delta}}=\|u\|_{S_{0}}$.

We will now show a nonlocal Poincaré-type inequality:

Lemma 3.4. There exists a $0<\tilde{\delta} \leq 1$ such that

$$
\|u\|_{L^{2}(\Omega)}^{2} \leq C B_{\delta}(u, u)
$$

for all $u \in S_{\delta}$ and $\delta \leq \tilde{\delta}$. Note that here $\tilde{\delta}$ depends on both $u$ and $\Omega$.

Proof. With Proposition 2 of [51], we have the bound for the first term in (2.7): there exist $\delta_{0}$ such that for all $\delta<\delta_{0}$,

$$
\|u\|_{L^{2}(\Omega)}^{2} \leq C^{*} b_{\delta}(u, u)
$$

and here we assume $C^{*}>0$ without loss of generality. To estimate the remaining two terms, we first work on the case where $\delta \Omega$ is a straight line. For $\mathbf{x} \in \Omega_{\delta}$ we have $M_{\delta}\left(\mathbf{x}_{l}\right) \frac{\left|\mathbf{r}^{\prime}(\mathbf{x})\right|}{\left|\mathbf{r}^{\prime}\left(\mathbf{x}_{l}\right)\right|}=M_{\delta}(\mathbf{x})$, and therefore the last term of $B_{\delta}(u, u)$ vanishes. For the second term of $B_{\delta}(u, u)$, with Lemma 3.1 we have $M_{\delta}(\mathbf{x}) \geq 0$, and therefore $h_{\delta}(u, u)=\int_{\Omega_{\delta}} M_{\delta}(\mathbf{x}) \int_{-\delta}^{\delta} H_{\delta}(|l|)\left[u\left(\mathbf{x}_{l}\right)-u(\mathbf{x})\right]^{2} \mathrm{~d} \mathbf{x}_{l} \mathrm{~d} \mathbf{x} \geq 0$. We then have the Poincaré-type inequality: there exists constants $C$ and $\delta_{0}$ such that for all $u \in S_{\delta}$ and $\delta \leq \delta_{0}$ :

$$
\|u\|_{L^{2}(\Omega)}^{2} \leq C^{*} b_{\delta}(u, u) \leq C^{*}\left(b_{\delta}(u, u)+h_{\delta}(u, u)\right)=C^{*} B_{\delta}(u, u) .
$$

We now proceed to finish the proof. Here we assume that $\|u\|_{L^{2}(\Omega)}>0$, otherwise the result is trivial. For simplicity, we now denote $\delta_{1}$ as $\min \left(\delta_{0}, \bar{\delta}\right)$ where $\bar{\delta}$ is defined in Lemma 3.1 and $\delta_{0}$ as in (3.13). With (3.13) and Lemma 3.1 we still have $\|u\|_{L^{2}(\Omega)}^{2} \leq C^{*} b_{\delta}(u, u)$ and $h_{\delta}(u, u) \geq 0$. We now proceed to estimate the last term in $B_{\delta}(u, u)$ :

$$
\begin{aligned}
& \int_{\Omega_{\delta}} \int_{-\delta}^{\delta}\left[M_{\delta}\left(\mathbf{x}_{l}\right) \frac{\left|\mathbf{r}^{\prime}(\mathbf{x})\right|}{\left|\mathbf{r}^{\prime}\left(\mathbf{x}_{l}\right)\right|}-M_{\delta}(\mathbf{x})\right] H_{\delta}(|l|)\left[u\left(\mathbf{x}_{l}\right)-u(\mathbf{x})\right] \mathrm{d} \mathbf{x}_{l} u(\mathbf{x}) \mathrm{d} \mathbf{x} \\
& \geq-\frac{1}{2} \int_{\Omega_{\delta}} M_{\delta}(\mathbf{x}) \int_{-\delta}^{\delta} H_{\delta}(|l|)\left[u\left(\mathbf{x}_{l}\right)-u(\mathbf{x})\right]^{2} \mathrm{~d} \mathbf{x}_{l} \mathrm{~d} \mathbf{x} \\
&-\frac{1}{2} \int_{\Omega_{\delta}} \int_{-\delta}^{\delta} H_{\delta}(|l|) \frac{\left|M_{\delta}(\mathbf{x})-M_{\delta}\left(\mathbf{x}_{l}\right)\right| \mathbf{r}^{\prime}(\mathbf{x})|/| \mathbf{r}^{\prime}\left(\mathbf{x}_{l}\right) \|^{2}}{\left|M_{\delta}(\mathbf{x})\right|} \mathrm{d} \mathbf{x}_{l}|u(\mathbf{x})|^{2} \mathrm{~d} \mathbf{x} \\
& \geq-\frac{1}{2} h_{\delta}(u, u)-\frac{C_{N} C_{M}}{2} \int_{\Omega_{\delta}} \int_{-\delta}^{\delta} H_{\delta}(|l|) \delta^{3} \mathrm{~d} \mathbf{x}_{l}|u(\mathbf{x})|^{2} \mathrm{~d} \mathbf{x} \\
& \geq-\frac{1}{2} h_{\delta}(u, u)-\frac{C_{N} C_{M} C_{H} \delta}{2}\|u\|_{L^{2}(\Omega)}^{2} .
\end{aligned}
$$


Hence, when

$$
\delta<\min \left\{\delta_{1}, \frac{1}{C^{*} C_{N} C_{M} C_{H}}\right\}=: \tilde{\delta}
$$

we have

$$
B_{\delta}(u, u) \geq\left(\frac{1}{C^{*}}-\frac{C_{N} C_{M} C_{H} \delta}{2}\right)\|u\|_{L^{2}(\Omega)}^{2}+\frac{1}{2} h_{\delta}(u, u) \geq \frac{1}{2 C^{*}}\|u\|_{L^{2}(\Omega)}^{2}
$$

The uniform boundedness of $L_{\delta}^{-1}$ then follows

Lemma 3.5. Assuming that $\Omega$ and $\tilde{\delta}$ satisfy the conditions in Lemma 3.4, there exists a constant $C$ such that

$$
\left\|L_{\delta}^{-1}\right\|_{L^{2}(\Omega)} \leq C .
$$

Moreover, with the definition of $\|\cdot\|_{S_{\delta}}$, we can show the boundedness and coercivity of the nonsymmetric bilinear operator $B_{\delta}(\cdot, \cdot)$ :

Lemma 3.6. There exists $a 0<\tilde{\delta} \leq 1$ such that for all $\delta<\tilde{\delta}$ the following inequalities hold

$$
\begin{aligned}
\forall u, v \in S_{\delta}, B_{\delta}(u, v) & \leq C_{1}\|u\|_{S_{\delta}}\|v\|_{S_{\delta}} \\
\forall u & \in S_{\delta}, \quad B_{\delta}(u, u) \geq C_{2}\|u\|_{S_{\delta}}^{2}
\end{aligned}
$$

for two constants $C_{1}, C_{2}>0$.

Proof. We first show (3.17). For the first two terms in $B_{\delta}(u, v)$, with the Cauchy-Schwarz inequality one may obtain $b_{\delta}(u, v) \leq C \sqrt{b_{\delta}(u, u) b_{\delta}(v, v)}$ and $h_{\delta}(u, v) \leq C \sqrt{h_{\delta}(u, u) h_{\delta}(v, v)}$. Moreover, with Lemma 3.1, similar as in (3.14) we can show that

$$
\begin{gathered}
\int_{\Omega_{\delta}} \int_{-\delta}^{\delta}\left[M_{\delta}\left(\mathbf{x}_{l}\right) \frac{\left|\mathbf{r}^{\prime}(\mathbf{x})\right|}{\left|\mathbf{r}^{\prime}\left(\mathbf{x}_{l}\right)\right|}-M_{\delta}(\mathbf{x})\right] H_{\delta}(|l|)\left[u\left(\mathbf{x}_{l}\right)-u(\mathbf{x})\right] \mathrm{d} \mathbf{x}_{l} v(\mathbf{x}) \mathrm{d} \mathbf{x} \\
\leq C \sqrt{h_{\delta}(u, u)\left(C_{N} C_{M} C_{H} \delta\|v\|_{L^{2}(\Omega)}^{2}\right)} \leq C \sqrt{h_{\delta}(u, u) b_{\delta}(v, v)} .
\end{gathered}
$$

Therefore

$$
B_{\delta}(u, v)^{2} \leq C\left(b_{\delta}(u, u) b_{\delta}(v, v)+h_{\delta}(u, u) b_{\delta}(v, v)+h_{\delta}(u, u) h_{\delta}(v, v)\right) \leq C\|u\|_{S_{\delta}}^{2}\|v\|_{S_{\delta}}^{2} .
$$

On the other hand, (3.18) can be obtained when $\tilde{\delta}$ is taken as in (3.15) and follow a similar proof as in Lemma 3.4.

With the above properties, we can see that there exists a unique solution $u_{\delta} \in S_{\delta}$ solving (2.6) (cf., [14], Thm. 2.5.6). The well-posedness of the proposed variational problem is therefore obtained. To further show the asymptotic property of solution when $\delta \rightarrow 0$, we need the following embedding property:

Lemma 3.7. For all $u \in S_{0}$ there exists a constant $C$ such that

$$
B_{\delta}(u, u) \leq C\|\nabla u\|_{L^{2}(\Omega)}^{2}
$$

for any $\delta$ satisfying the condition in Lemma 3.6. 
Proof. Given $u \in S_{0}$, from Theorem 1 of [13] we have that

$$
b_{\delta}(u, u) \leq C\|u\|_{H^{1}(\Omega)}^{2} \leq C\|\nabla u\|_{L^{2}(\Omega)}^{2} .
$$

To bound the second and the third terms of $B_{\delta}(u, u)$, we start with the case of boundary curvature $\equiv 0$, where we only need to show that $h_{\delta}(u, u)=\int_{\Omega_{\delta}} M_{\delta}(\mathbf{x}) \int_{-\delta}^{\delta} H_{\delta}(|l|)\left[u\left(\mathbf{x}_{l}\right)-u(\mathbf{x})\right]^{2} \mathrm{~d} \mathbf{x}_{l} \mathrm{~d} \mathbf{x} \leq C\|\nabla u\|_{L^{2}(\Omega)}^{2}$. Since $M_{\delta}(\mathbf{x}) \leq C$, it suffices to estimate $\int_{\Omega_{\delta}} \int_{-\delta}^{\delta} H_{\delta}(|l|)\left[u\left(\mathbf{x}_{l}\right)-u(\mathbf{x})\right]^{2} \mathrm{~d} \mathbf{x}_{l} \mathrm{~d} \mathbf{x}$. With the Hölder inequality and the fact that $\int_{\Omega_{\delta}}\left|\nabla u\left(\mathbf{x}_{t}\right)\right|^{2} \mathrm{~d} \mathbf{x}=\int_{\Omega_{\delta}}|\nabla u(\mathbf{x})|^{2} \mathrm{~d} \mathbf{x}$ for all $|t| \leq \delta$, we have

$$
\begin{aligned}
& \int_{\Omega_{\delta}} \int_{-\delta}^{\delta} H_{\delta}(|l|)\left[u\left(\mathbf{x}_{l}\right)-u(\mathbf{x})\right]^{2} \mathrm{~d} \mathbf{x}_{l} \mathrm{~d} \mathbf{x} \\
& \quad \leq \sup _{r \leq 1} H(|r|) \frac{1}{\delta^{3}} \int_{\Omega_{\delta}} \int_{-\delta}^{\delta}\left[u\left(\mathbf{x}_{l}\right)-u(\mathbf{x})\right]^{2} \mathrm{~d} \mathbf{x}_{l} \mathrm{~d} \mathbf{x} \leq \frac{C}{\delta^{2}} \int_{\Omega_{\delta}} \int_{-\delta}^{\delta} \int_{0}^{l}\left|\nabla u\left(\mathbf{x}_{t}\right)\right|^{2} \mathrm{~d} t \mathrm{~d} \mathbf{x}_{l} \mathrm{~d} \mathbf{x} \\
& \quad \leq \frac{C}{\delta^{2}} \int_{\Omega_{\delta}} \int_{-\delta}^{\delta} \int_{0}^{\delta}\left|\nabla u\left(\mathbf{x}_{t}\right)\right|^{2} \mathrm{~d} t \mathrm{~d} \mathbf{x}_{l} \mathrm{~d} \mathbf{x}=\frac{C}{\delta} \int_{\Omega_{\delta}} \int_{0}^{\delta}\left|\nabla u\left(\mathbf{x}_{t}\right)\right|^{2} \mathrm{~d} t \mathrm{~d} \mathbf{x} \\
& \quad=\frac{C}{\delta} \int_{0}^{\delta} \int_{\Omega_{\delta}}\left|\nabla u\left(\mathbf{x}_{t}\right)\right|^{2} \mathrm{~d} \mathbf{x} \mathrm{d} t=C \int_{\Omega_{\delta}}|\nabla u(\mathbf{x})|^{2} \mathrm{~d} \mathbf{x} \leq C\|\nabla u\|_{L^{2}(\Omega)}^{2} .
\end{aligned}
$$

Therefore, the Lemma holds true when the boundary curvature $\kappa(\mathbf{x}) \equiv 0$, a.e. We now work on the case of nonzero curvature. Similar as in the curvature $\equiv 0$ case we can obtain $h_{\delta}(u, u) \leq C\|\nabla u\|_{L^{2}(\Omega)}^{2}$. For the last term of $B_{\delta}(u, u)$, with (3.19) we have

$$
\begin{aligned}
& \int_{\Omega_{\delta}} \int_{-\delta}^{\delta}\left[M_{\delta}\left(\mathbf{x}_{l}\right) \frac{\left|\mathbf{r}^{\prime}(\mathbf{x})\right|}{\left|\mathbf{r}^{\prime}\left(\mathbf{x}_{l}\right)\right|}-M_{\delta}(\mathbf{x})\right] H_{\delta}(|l|)\left[u\left(\mathbf{x}_{l}\right)-u(\mathbf{x})\right] \mathrm{d} \mathbf{x}_{l} u(\mathbf{x}) \mathrm{d} \mathbf{x} \\
& \leq C \sqrt{h_{\delta}(u, u) b_{\delta}(u, u)} \leq C\|\nabla u\|_{L^{2}(\Omega)}^{2} .
\end{aligned}
$$

Before studying the limiting behavior of the nonlocal operator, we need a compactness property:

Lemma 3.8. Suppose $u_{n} \in S_{\delta_{n}}$ and $\delta_{n} \rightarrow 0$, then given $\sup _{n} B_{\delta_{n}}\left(u_{n}, u_{n}\right) \leq \infty$, $u_{n}$ is precompact in $L^{2}(\Omega)$. Moreover, any limit point $u \in S_{0}$.

Proof. Since $S_{\delta_{n}} \subseteq L^{2}(\Omega)$ and $h_{\delta}\left(u_{n}, u_{n}\right) \geq 0$, similar to (3.14) we have,

$$
B_{\delta}(u, u) \geq \frac{1}{2} \int_{\Omega} \int_{\Omega} J_{\delta_{n}}(\mathbf{x}-\mathbf{y})\left(u_{n}(\mathbf{y})-u_{n}(\mathbf{x})\right)^{2} \mathrm{~d} \mathbf{y} \mathrm{d} \mathbf{x}+\left(\frac{1}{2 C^{*}}-\frac{C_{N} C_{M} C_{H} \delta}{2}\right)\|u\|_{L^{2}(\Omega)}^{2}
$$

where $C^{*}$ denotes the constant in (3.13). Therefore, when $\tilde{\delta}$ is taken as in (3.15), then for all $\delta<\tilde{\delta}$

$$
B_{\delta_{n}}\left(u_{n}, u_{n}\right) \geq \frac{1}{2} \int_{\Omega} \int_{\Omega} J_{\delta_{n}}(\mathbf{x}-\mathbf{y})\left(u_{n}(\mathbf{y})-u_{n}(\mathbf{x})\right)^{2} \mathrm{~d} \mathbf{y} \mathrm{d} \mathbf{x} .
$$

We have $u_{n} \in L^{2}(\Omega)$ and

$$
\int_{\Omega} \int_{\Omega} J_{\delta_{n}}(\mathbf{x}-\mathbf{y})\left(u_{n}(\mathbf{y})-u_{n}(\mathbf{x})\right)^{2} \mathrm{~d} \mathbf{y} \mathrm{d} \mathbf{x} \leq \infty
$$

From Theorem 1.2 of [56], any limit of $\left\{u_{n}\right\}$ is in $L^{2}(\Omega)$, or equivalently, $u_{n}$ is precompact, and any limit point $u \in S_{0}$. 
With the above lemmas, we obtain the following $L^{2}$ convergence result for an intermediate solution as $\delta \rightarrow 0$ :

Lemma 3.9. Suppose $\tilde{u}_{\delta}$ is the weak solution of

$$
\left\{\begin{array}{l}
L_{N \delta} \tilde{u}_{\delta}=f, \text { in } \Omega \\
\int_{\Omega} \tilde{u}_{\delta} \mathrm{d} \mathbf{x}=0
\end{array}\right.
$$

and $u_{0}$ is the weak solution of (2.1), then

$$
\lim _{\delta \rightarrow 0}\left\|\tilde{u}_{\delta}-u_{0}\right\|_{L^{2}(\Omega)}=0 .
$$

Proof. The proof follows a similar strategy as in [61,63]. A detailed derivation is provided in Appendix A.1.

We now have the main theorem of this section for $f \in C(\bar{\Omega})$ :

Theorem 3.10. Suppose $u_{\delta}$ is the weak solution of (2.5) and $u_{0}$ is the weak solution of (2.1), then

$$
\lim _{\delta \rightarrow 0}\left\|u_{\delta}-u_{0}\right\|_{L^{2}(\Omega)}=0 .
$$

Proof. With the results in Lemma 3.9, we only need to show that $\lim _{\delta \rightarrow 0}\left\|u_{\delta}-\tilde{u}_{\delta}\right\|_{L^{2}(\Omega)}=0$. Since $L_{\delta}\left(u_{\delta}-\tilde{u}_{\delta}\right)=$ $f_{\delta}-f$, with Lemma 3.5 we can see that it suffices to show

$$
\lim _{\delta \rightarrow 0}\left\|f_{\delta}-f\right\|_{L^{2}(\Omega)}=0,
$$

or equivalently

$$
\lim _{\delta \rightarrow 0} \int_{\Omega_{\delta}}\left(\int_{\left(\mathbb{R}^{N} \backslash \Omega\right)_{\delta}} J_{\delta}(|\mathbf{x}-\mathbf{y}|)\left(|(\mathbf{y}-\overline{\mathbf{x}}) \cdot \mathbf{n}(\overline{\mathbf{x}})|^{2}-|(\mathbf{x}-\overline{\mathbf{x}}) \cdot \mathbf{n}(\overline{\mathbf{x}})|^{2}\right) \mathrm{d} \mathbf{y} f(\mathbf{x})\right)^{2} \mathrm{~d} \mathbf{x}=0 .
$$

Since

$$
\begin{gathered}
\left|\int_{\left(\mathbb{R}^{N} \backslash \Omega\right)_{\delta}} J_{\delta}(|\mathbf{x}-\mathbf{y}|)\left(|(\mathbf{y}-\overline{\mathbf{x}}) \cdot \mathbf{n}(\overline{\mathbf{x}})|^{2}-|(\mathbf{x}-\overline{\mathbf{x}}) \cdot \mathbf{n}(\overline{\mathbf{x}})|^{2}\right) \mathrm{d} \mathbf{y}\right| \\
\leq C \int_{\left(\mathbb{R}^{N} \backslash \Omega\right)_{\delta}} J_{\delta}(|\mathbf{x}-\mathbf{y}|)|(\mathbf{y}-\mathbf{x}) \cdot \mathbf{n}(\overline{\mathbf{x}})|^{2} \mathrm{~d} \mathbf{y} \leq C
\end{gathered}
$$

we have

$$
\int_{\Omega_{\delta}}\left(\int_{\left(\mathbb{R}^{N} \backslash \Omega\right)_{\delta}} J_{\delta}(|\mathbf{x}-\mathbf{y}|)\left(((\mathbf{y}-\overline{\mathbf{x}}) \cdot \mathbf{n}(\overline{\mathbf{x}}))^{2}-((\mathbf{x}-\overline{\mathbf{x}}) \cdot \mathbf{n}(\overline{\mathbf{x}}))^{2}\right) \mathrm{d} \mathbf{y} f(\mathbf{x})\right)^{2} \mathrm{~d} \mathbf{x} \leq C \int_{\Omega_{\delta}}|f(\mathbf{x})|^{2} \mathrm{~d} \mathbf{x}
$$

which vanishes as $\delta \rightarrow 0$.

Remark 3.11. For the analysis in this paper we focus on the homogeneous Neumann-type boundary condition $g(\mathbf{x})=0$, while we note that the proposed nonlocal variational formulation can be applied to inhomogeneous boundary conditions. Here we take $J_{\delta}(r)=\frac{4}{\pi \delta^{4}}$ for simplicity. When $f(\mathbf{x})=0$ and $g(\mathbf{x}) \neq 0$, applying a test function $v(\mathbf{x}) \in C^{\infty}(\Omega)$ to (2.4) yields

$$
\begin{aligned}
\left(f_{\delta}, v\right)_{L^{2}(\Omega)} & =\int_{\Omega}\left(2 \int_{\mathbb{R}^{N} \backslash \Omega} J_{\delta}(|\mathbf{x}-\mathbf{y}|)(\mathbf{y}-\mathbf{x}) \cdot \mathbf{n}(\overline{\mathbf{x}}) \mathrm{d} \mathbf{y}-M_{\delta}(\mathbf{x}) \kappa(\overline{\mathbf{x}})\right) g(\overline{\mathbf{x}}) v(\mathbf{x}) \mathrm{d} \mathbf{x} \\
& =2 \int_{\Omega_{\delta}} \int_{\mathbb{R}^{N} \backslash \Omega} J_{\delta}(|\mathbf{x}-\mathbf{y}|)(\mathbf{y}-\mathbf{x}) \cdot \mathbf{n}(\overline{\mathbf{x}}) \mathrm{d} \mathbf{y} g(\overline{\mathbf{x}}) v(\mathbf{x}) \mathrm{d} \mathbf{x}-\int_{\Omega_{\delta}} M_{\delta}(\mathbf{x}) \kappa(\overline{\mathbf{x}}) g(\overline{\mathbf{x}}) v(\mathbf{x}) \mathrm{d} \mathbf{x} .
\end{aligned}
$$


For the second part, with the Hölder inequality we have

$$
\left|\int_{\Omega_{\delta}} M_{\delta}(\mathbf{x}) \kappa(\overline{\mathbf{x}}) g(\overline{\mathbf{x}}) v(\mathbf{x}) \mathrm{d} \mathbf{x}\right| \leq C \sqrt{\int_{\Omega_{\delta}} g^{2}(\overline{\mathbf{x}}) \mathrm{d} \mathbf{x} \int_{\Omega_{\delta}} v^{2}(\mathbf{x}) \mathrm{d} \mathbf{x}} \leq C \sqrt{\delta\|g\|_{L^{2}(\partial \Omega)}\|v\|_{L^{2}(\Omega)}} .
$$

Therefore, $\left|\int_{\Omega_{\delta}} M_{\delta}(\mathbf{x}) \kappa(\overline{\mathbf{x}}) g(\overline{\mathbf{x}}) v(\mathbf{x}) \mathrm{d} \mathbf{x}\right| \rightarrow 0$ as $\delta \rightarrow 0$. To show the asymptotic limit for the first part as $\delta \rightarrow 0$, for each $\mathbf{x} \in \Omega_{\delta}$ we have

$$
\int_{\mathbb{R}^{N} \backslash \Omega} J_{\delta}(|\mathbf{x}-\mathbf{y}|)(\mathbf{y}-\mathbf{x}) \cdot \mathbf{n}(\overline{\mathbf{x}}) \mathrm{d} \mathbf{y}=\int_{D_{\delta}} J_{\delta}(|\mathbf{x}-\mathbf{y}|)(\mathbf{y}-\mathbf{x}) \cdot \mathbf{n}(\overline{\mathbf{x}}) \mathrm{d} \mathbf{y}+\int_{A_{\delta}} J_{\delta}(|\mathbf{x}-\mathbf{y}|)(\mathbf{y}-\mathbf{x}) \cdot \mathbf{n}(\overline{\mathbf{x}}) \mathrm{d} \mathbf{y} .
$$

For the first part

$$
\int_{D_{\delta}} J_{\delta}(|\mathbf{x}-\mathbf{y}|)(\mathbf{y}-\mathbf{x}) \cdot \mathbf{n}(\overline{\mathbf{x}}) \mathrm{d} \mathbf{y}=\frac{4}{\pi \delta^{4}} \int_{s_{x}}^{\delta} 2 r \sqrt{\delta^{2}-r^{2}} \mathrm{~d} r=\frac{8}{3 \pi \delta^{4}}\left(\delta^{2}-s_{x}^{2}\right)^{3 / 2}
$$

and

$$
\begin{aligned}
& 2 \int_{\Omega_{\delta}} \int_{D_{\delta}} J_{\delta}(|\mathbf{x}-\mathbf{y}|)(\mathbf{y}-\mathbf{x}) \cdot \mathbf{n}(\overline{\mathbf{x}}) \mathrm{d} \mathbf{y} g(\overline{\mathbf{x}}) v(\mathbf{x}) \mathrm{d} \mathbf{x} \\
& \quad=\frac{16}{3 \pi \delta^{4}} \int_{\Omega_{\delta}}\left(\delta^{2}-s_{x}^{2}\right)^{3 / 2} g(\overline{\mathbf{x}}) v(\mathbf{x}) \mathrm{d} \mathbf{x}=\frac{16}{3 \pi \delta^{4}} \int_{\partial \Omega} \int_{0}^{\delta}\left(\delta^{2}-r^{2}\right)^{3 / 2} \mathrm{~d} r g(\overline{\mathbf{x}}) v(\overline{\mathbf{x}}) \mathrm{d} \overline{\mathbf{x}}+\mathrm{O}(\delta) \\
& \quad=\int_{\partial \Omega} g(\overline{\mathbf{x}}) v(\overline{\mathbf{x}}) \mathrm{d} \overline{\mathbf{x}}+\mathrm{O}(\delta) .
\end{aligned}
$$

For the second part, since the area of $A_{\delta}$ is bounded by $C \delta^{3}$, we have

$$
\left|\int_{A_{\delta}} J_{\delta}(|\mathbf{x}-\mathbf{y}|)(\mathbf{y}-\mathbf{x}) \cdot \mathbf{n}(\overline{\mathbf{x}}) \mathrm{d} \mathbf{y}\right| \leq C \delta .
$$

Combining (3.25) and (3.26) yields

$$
\lim _{\delta \rightarrow 0}\left(f_{\delta}, v\right)_{L^{2}(\Omega)}=\lim _{\delta \rightarrow 0} 2 \int_{\Omega_{\delta}} \int_{\mathbb{R}^{N} \backslash \Omega} J_{\delta}(|\mathbf{x}-\mathbf{y}|)(\mathbf{y}-\mathbf{x}) \cdot \mathbf{n}(\overline{\mathbf{x}}) \mathrm{d} \mathbf{y} g(\overline{\mathbf{x}}) v(\mathbf{x}) \mathrm{d} \mathbf{x}=\int_{\partial \Omega} g(\overline{\mathbf{x}}) v(\overline{\mathbf{x}}) \mathrm{d} \overline{\mathbf{x}} .
$$

Therefore, the right hand side converges to the inhomogeneous flux condition as $\delta \rightarrow 0$ in the variational formulation. In fact, the asymptotic convergence property in Theorem 3.10 can be shown for the nonlocal diffusion problem with inhomogeneous flux conditions given the corresponding nonlocal trace theorem, which will be addressed in the future work.

\section{Convergence Rate in the $L^{\infty}(\Omega)$ norm}

In this section, we will estimate the order of convergence rate by considering a problem with the more general setting: $\partial \Omega=\partial \Omega_{D} \bigcup \partial \Omega_{N}$ and $\left(\partial \Omega_{D}\right)^{\circ} \bigcap\left(\partial \Omega_{N}\right)^{\circ}=\emptyset$. Here $\partial \Omega_{D}$ and $\partial \Omega_{N}$ are both $1 \mathrm{D}$ curves. To define a Dirichlet-type constraint on $\partial \Omega_{D}$, we denote $\Omega_{D \delta}=\left\{\mathbf{x} \in \Omega_{\delta}: \overline{\mathbf{x}} \in \partial \Omega_{D}\right\}$ where $\overline{\mathbf{x}}$ is the orthogonal projection of $\mathbf{x}$ on $\partial \Omega$. Moreover, we denote $\partial \Omega_{D \delta}=\left\{\mathbf{x} \in \mathbb{R}^{N} \backslash \Omega\right.$ : $\left.\operatorname{dist}\left(\mathbf{x}, \Omega_{D \delta}\right) \leq \delta\right\}$ and assume that the value of $u$ is given on it. To be specific, here we assume $u(\mathbf{x})=0$ on $\partial \Omega_{D \delta}$ without loss of generality. Similarly, to apply the Neumanntype constraint on $\partial \Omega_{N}$, we denote $\Omega_{N \delta}=\left\{\mathbf{x} \in \Omega_{\delta}: \overline{\mathbf{x}} \in \partial \Omega_{N}\right\}$ and $\partial \Omega_{N \delta}=\left\{\mathbf{x} \in \mathbb{R}^{N} \backslash \Omega: \operatorname{dist}\left(\mathbf{x}, \Omega_{N \delta}\right) \leq \delta\right\}$. We consider a Neumann-type constraint as an extension of $\frac{\partial u}{\partial \mathbf{n}}=g(\mathbf{x})$ on $\partial \Omega_{N}$, by modifying the nonlocal problem discussed in the last section as follows: for $\mathbf{x} \in \Omega \backslash \Omega_{N \delta}$ :

$$
-2 \int_{\Omega \cup \partial \Omega_{D \delta}} J_{\delta}(|\mathbf{x}-\mathbf{y}|)\left(u_{\delta}(\mathbf{y})-u_{\delta}(\mathbf{x})\right) \mathrm{d} \mathbf{y}=f(\mathbf{x}),
$$


and for $\mathbf{x} \in \Omega_{N \delta}$ :

$$
\begin{aligned}
& -2 \int_{\Omega} J_{\delta}(|\mathbf{x}-\mathbf{y}|)\left(u_{\delta}(\mathbf{y})-u_{\delta}(\mathbf{x})\right) \mathrm{d} \mathbf{y}-2 M_{\delta}(\mathbf{x}) \int_{-\delta}^{\delta} H_{\delta}(|l|)\left(u_{\delta}\left(\mathbf{x}_{l}\right)-u_{\delta}(\mathbf{x})\right) \mathrm{d} \mathbf{x}_{l} \\
& =f(\mathbf{x})-\int_{\partial \Omega_{N \delta}} J_{\delta}(|\mathbf{x}-\mathbf{y}|)\left[|(\mathbf{y}-\overline{\mathbf{x}}) \cdot \mathbf{n}(\overline{\mathbf{x}})|^{2}-|(\mathbf{x}-\overline{\mathbf{x}}) \cdot \mathbf{n}(\overline{\mathbf{x}})|^{2}\right] \mathrm{d} \mathbf{y} f(\mathbf{x}) \\
& \quad+\left(2 \int_{\partial \Omega_{N \delta}} J_{\delta}(|\mathbf{x}-\mathbf{y}|)(\mathbf{y}-\mathbf{x}) \cdot \mathbf{n}(\overline{\mathbf{x}}) \mathrm{d} \mathbf{y}-M_{\delta}(\mathbf{x}) \kappa(\overline{\mathbf{x}})\right) g(\overline{\mathbf{x}})
\end{aligned}
$$

where

$$
M_{\delta}(\mathbf{x}):=\int_{\partial \Omega_{N \delta}} J_{\delta}(|\mathbf{x}-\mathbf{y}|)\left[|(\mathbf{y}-\mathbf{x}) \cdot \mathbf{p}(\overline{\mathbf{x}})|^{2}-|(\mathbf{y}-\overline{\mathbf{x}}) \cdot \mathbf{n}(\overline{\mathbf{x}})|^{2}+|(\mathbf{x}-\overline{\mathbf{x}}) \cdot \mathbf{n}(\overline{\mathbf{x}})|^{2}\right] \mathrm{d} \mathbf{y} .
$$

Here we note that it is possible that $\Omega_{D \delta} \bigcap \Omega_{N \delta} \neq \emptyset$. We can then rewrite the nonlocal equation to be solved as

$$
\begin{cases}L_{\delta} u=f, & \text { on } \Omega \backslash \Omega_{N \delta} \\ L_{N \delta} u=f_{\delta}, & \text { on } \Omega_{N \delta} \\ u=0, & \text { on } \partial \Omega_{D \delta} .\end{cases}
$$

The corresponding limiting local model is given by

$$
\begin{cases}-\triangle u=f, & \text { on } \Omega \\ \frac{\partial u}{\partial \mathbf{n}}=g, & \text { on } \partial \Omega_{N} \\ u=0, & \text { on } \partial \Omega_{D} .\end{cases}
$$

Here $f(\mathbf{x})$ is the given data corresponding to source, $g(\mathbf{x})$ is the given local flux on $\partial \Omega_{N}$, and

$$
\begin{aligned}
f_{\delta}(\mathbf{x}):= & f(\mathbf{x})-\int_{\partial \Omega_{N \delta}} J_{\delta}(|\mathbf{x}-\mathbf{y}|)\left[|(\mathbf{y}-\overline{\mathbf{x}}) \cdot \mathbf{n}(\overline{\mathbf{x}})|^{2}-|(\mathbf{x}-\overline{\mathbf{x}}) \cdot \mathbf{n}(\overline{\mathbf{x}})|^{2}\right] \mathrm{d} \mathbf{y} f(\mathbf{x}) \\
& +\left(2 \int_{\partial \Omega_{N \delta}} J_{\delta}(|\mathbf{x}-\mathbf{y}|)(\mathbf{y}-\mathbf{x}) \cdot \mathbf{n}(\overline{\mathbf{x}}) \mathrm{d} \mathbf{y}-M_{\delta}(\mathbf{x}) \kappa(\overline{\mathbf{x}})\right) g(\overline{\mathbf{x}}) .
\end{aligned}
$$

In this section, we focus on the case with homogeneous Neumann-type constraints, i.e., $g(\mathbf{x})=0$.

For the above problem with mixed constraints, we have the nonlocal maximum principle stated below

Lemma 4.1. For $u \in C(\bar{\Omega}) \cap C\left(\partial \Omega_{D \delta} \backslash \partial \Omega_{D}\right)$ and $u$ bounded on $\partial \Omega_{D \delta}$, assuming that $u$ satisfies $L_{\delta} u \leq 0$ for all $x \in \Omega \backslash \Omega_{N \delta}$ and $L_{N \delta} u \leq 0$ for all $x \in \Omega_{N \delta}$, we have

$$
\sup _{\mathbf{x} \in \bar{\Omega} \cup \partial \Omega_{D \delta}} u(\mathbf{x}) \leq \sup _{\mathbf{x} \in \partial \Omega_{D \delta}} u(\mathbf{x})
$$

Proof. Assuming that $\sup _{\mathbf{x} \in \bar{\Omega} \cup \partial \Omega_{D \delta}} u(\mathbf{x})>\sup _{\mathbf{x} \in \partial \Omega_{D \delta}} u(\mathbf{x})$, since $u \in C(\bar{\Omega})$ there exists $\mathbf{x}^{*} \in\left(\Omega \cup \partial \Omega_{N}\right)$ such that $u\left(\mathbf{x}^{*}\right)=\sup _{\mathbf{x} \in\left(\bar{\Omega} \cup \partial \Omega_{D \delta}\right)} u(\mathbf{x})$.

Case 1. $\mathbf{x}^{*} \in \Omega \backslash \Omega_{N \delta}$. Then $L_{\delta} u\left(\mathbf{x}^{*}\right)=-2 \int_{\Omega \cup \partial \Omega_{D \delta}} J_{\delta}\left(\left|\mathbf{x}^{*}-\mathbf{y}\right|\right)\left(u(\mathbf{y})-u\left(\mathbf{x}^{*}\right)\right) \mathrm{d} \mathbf{y} \geq 0$. Therefore $L_{\delta} u\left(\mathbf{x}^{*}\right)=0$ and

$$
u(\mathbf{y})=u\left(\mathbf{x}^{*}\right)=\sup _{\mathbf{x} \in \bar{\Omega} \cup \partial \Omega_{D \delta}} u(\mathbf{x}), \quad \forall \mathbf{y} \in\left(\bar{\Omega} \cup \partial \Omega_{D \delta}\right) \cap B\left(\mathbf{x}^{*}, \delta\right) .
$$

Case 2. $\mathbf{x}^{*} \in \Omega_{N \delta}$. Then

$$
L_{N \delta} u\left(\mathbf{x}^{*}\right)=-2 \int_{\Omega} J_{\delta}\left(\left|\mathbf{x}^{*}-\mathbf{y}\right|\right)\left(u(\mathbf{y})-u\left(\mathbf{x}^{*}\right)\right) \mathrm{d} \mathbf{y}-2 M_{\delta}\left(\mathbf{x}^{*}\right) \int_{-\delta}^{\delta} H(|l|)\left[u\left(\mathbf{x}_{l}^{*}\right)-u\left(\mathbf{x}^{*}\right)\right] \mathrm{d} \mathbf{x}_{l}^{*} \geq 0 .
$$


Note that in Lemma 3.1 we have proven $M_{\delta}\left(\mathbf{x}^{*}\right) \geq 0$. Again, this is possible only when

$$
u(\mathbf{y})=u\left(\mathbf{x}^{*}\right)=\sup _{\mathbf{x} \in \bar{\Omega} \cup \partial \Omega_{D \delta}} u(\mathbf{x}), \quad \forall \mathbf{y} \in \bar{\Omega} \cap B\left(\mathbf{x}^{*}, \delta\right) .
$$

Summing up the two cases, in view of (4.5) and (4.6), we have

$$
\begin{gathered}
\mathbf{x}^{*} \in \Omega \backslash \Omega_{N \delta} \Rightarrow u(\mathbf{y})=u\left(\mathbf{x}^{*}\right)=\sup _{\bar{\Omega} \cup \partial \Omega_{D \delta}} u, \forall \mathbf{y} \in\left(\bar{\Omega} \cup \partial \Omega_{D \delta}\right) \cap B\left(\mathbf{x}^{*}, \delta\right), \\
\mathbf{x}^{*} \in \Omega_{N \delta} \Rightarrow u(\mathbf{y})=u\left(\mathbf{x}^{*}\right)=\sup _{\bar{\Omega} \cup \partial \Omega_{D \delta}} u, \forall \mathbf{y} \in \bar{\Omega} \cap B\left(\mathbf{x}^{*}, \delta\right) .
\end{gathered}
$$

Now fixing $\mathbf{y}^{*} \in\left(\left(\Omega \cup \partial \Omega_{N}\right) \cap B\left(\mathbf{x}^{*}, \delta\right)\right)$, we can apply the same arguments with $\mathbf{y}^{*}$ in place of $\mathbf{x}^{*}$, and get $(4.7)$ and (4.8) with $\mathbf{y}^{*}$ in the role of $\mathbf{x}^{*}$. This process can be repeated for all points $\mathbf{y}^{*} \in\left(\left(\Omega \cup \partial \Omega_{N}\right) \cap B\left(\mathbf{x}^{*}, \delta\right)\right)$, and together with the continuity assumption of $u$ we obtain:

$$
u(\mathbf{y})=u\left(\mathbf{x}^{*}\right)=\sup _{\bar{\Omega} \cup \partial \Omega_{D \delta}} u, \quad \forall \mathbf{y} \in\left(\bar{\Omega} \cup \partial \Omega_{D \delta}\right) \cap\left[B\left(\mathbf{x}^{*}, \delta\right) \cup\left(\bigcup_{\mathbf{y}^{*} \in\left(\Omega \cup \partial \Omega_{N}\right) \cap B\left(\mathbf{x}^{*}, \delta\right)} B\left(\mathbf{y}^{*}, \delta\right)\right)\right] .
$$

Geometrically, note that

$$
\begin{array}{r}
\left(\bar{\Omega} \cup \partial \Omega_{D \delta}\right) \cap\left[B\left(\mathbf{x}^{*}, \delta\right) \cup\left(\bigcup_{\mathbf{y}^{*} \in\left(\Omega \cup \partial \Omega_{N}\right) \cap B\left(\mathbf{x}^{*}, \delta\right)} B\left(\mathbf{y}^{*}, \delta\right)\right)\right] \\
=\left\{z \in \bar{\Omega} \cup \partial \Omega_{D \delta}: \operatorname{dist}\left(z,\left(\Omega \cup \partial \Omega_{N}\right) \cap B\left(\mathbf{x}^{*}, \delta\right)\right) \leq \delta\right\} .
\end{array}
$$

In other words, with this argument we expanded the region where $u(\mathbf{z})=\sup _{\bar{\Omega} \cup \partial \Omega_{D \delta}} u$ from $\mathbf{z} \in\left(\Omega \cup \partial \Omega_{N}\right) \cap$ $B\left(\mathbf{x}^{*}, \delta\right)$ to its entire $\delta$-neighborhood lying in $\bar{\Omega} \cup \partial \Omega_{D \delta}$. We then apply this argument recursively, so that the region where $u(\mathbf{z})=\sup _{\bar{\Omega} \cup \partial \Omega_{D \delta}} u$ will get expanded to the entire domain of $\bar{\Omega} \cup \partial \Omega_{D \delta}$. In other words, to have a global maximum inside $\Omega$, the only possibility is for $u$ to be constant on $\bar{\Omega} \cup \partial \Omega_{D \delta}$, which contradicts with the assumption that $\sup _{\mathbf{x} \in \bar{\Omega} \cup \partial \Omega_{D \delta}} u(\mathbf{x})>\sup _{\mathbf{x} \in \partial \Omega_{D \delta}} u(\mathbf{x})$.

We now assume that $u_{\delta}$ is the solution of (4.2) and $u_{0}$ is the solution of (4.3). Denote $e_{\delta}(\mathbf{x}):=u_{\delta}(\mathbf{x})-u_{0}(\mathbf{x})$, $T_{\delta}(\mathbf{x}):=\left(L_{0} u_{0}(\mathbf{x})-L_{\delta} u_{0}(\mathbf{x})\right)+\left(f_{\delta}(\mathbf{x})-f(\mathbf{x})\right)$ for $\mathbf{x} \in \Omega \backslash \Omega_{N \delta}$ and $T_{\delta}(\mathbf{x}):=\left(L_{0} u_{0}(\mathbf{x})-L_{N \delta} u_{0}(\mathbf{x})\right)+\left(f_{\delta}(\mathbf{x})-f(\mathbf{x})\right)$ for $\mathbf{x} \in \Omega_{N \delta}$, then for $\mathbf{x} \in \Omega \backslash \Omega_{N \delta}$,

$$
L_{\delta} e_{\delta}=L_{\delta} u_{\delta}-L_{\delta} u_{0}=L_{0} u_{0}-L_{\delta} u_{0}=T_{\delta},
$$

and similarly for $\mathbf{x} \in \Omega_{N \delta}$,

$$
L_{\delta} e_{\delta}=L_{N \delta} u_{\delta}-L_{N \delta} u_{0}=f_{\delta}-f+L_{0} u_{0}-L_{N \delta} u_{0}=T_{\delta} .
$$

We then obtain the following truncation estimate for $T_{\delta}$ :

Lemma 4.2. Suppose $u_{0}$ is the solution to local problem (4.3), then

$$
T_{\delta}(\mathbf{x})=O\left(\delta^{2}\right)
$$

for $\mathbf{x} \in \Omega \backslash \Omega_{N \delta}$, and

$$
\begin{aligned}
T_{\delta}(\mathbf{x})= & 2 \int_{E_{\delta}} J_{\delta}(|\mathbf{x}-\mathbf{y}|) \frac{\partial u_{0}(\mathbf{x})}{\partial \mathbf{p}}((\mathbf{x}-\mathbf{y}) \cdot \mathbf{p}(\overline{\mathbf{x}})) \mathrm{d} \mathbf{y} \\
& +\int_{\Omega} J_{\delta}(|\mathbf{x}-\mathbf{y}|)\left[u_{0}(\mathbf{x})\right]_{n n n}((\mathbf{x}-\mathbf{y}) \cdot \mathbf{n}(\overline{\mathbf{x}}))\left(-|\overline{\mathbf{x}}-\mathbf{x}|^{2}+\frac{1}{3}|(\mathbf{x}-\mathbf{y}) \cdot \mathbf{n}(\overline{\mathbf{x}})|^{2}\right) \mathrm{d} \mathbf{y} \\
& +\int_{\Omega} J_{\delta}(|\mathbf{x}-\mathbf{y}|)\left[u_{0}(\mathbf{x})\right]_{n p p}((\mathbf{x}-\mathbf{y}) \cdot \mathbf{n}(\overline{\mathbf{x}}))|(\mathbf{x}-\mathbf{y}) \cdot \mathbf{p}(\overline{\mathbf{x}})|^{2} \mathrm{~d} \mathbf{y} \\
& +\kappa(\overline{\mathbf{x}}) M_{\delta}(\mathbf{x})\left[u_{0}(\mathbf{x})\right]_{n n}((\mathbf{x}-\overline{\mathbf{x}}) \cdot \mathbf{n}(\overline{\mathbf{x}}))+O\left(\delta^{2}\right)
\end{aligned}
$$




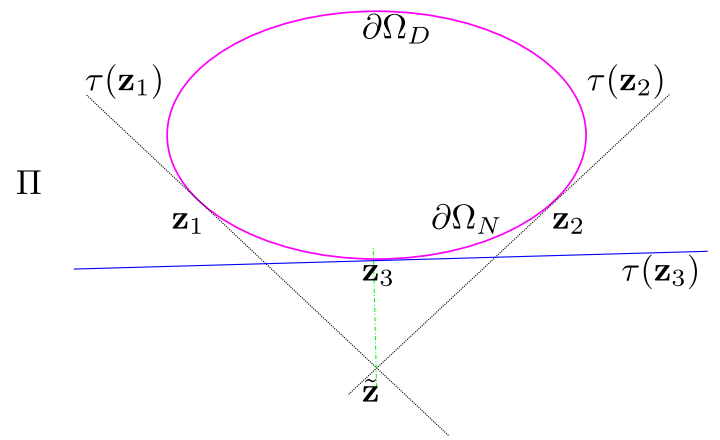

FIGURE 3. Illustration of the geometric assumption and notation for the barrier function $\phi(\mathbf{x})$ definition.

for $\mathbf{x} \in \Omega_{N \delta}$. Here $E_{\delta}$ denotes the region in $A_{\delta}$ which is asymmetric with respect to the $y$ axis (see the right plot of Fig. 2).

Proof. The proof is based on the Taylor expansion of $u_{0}$ and an estimate for the asymmetric part in $A_{\delta}$. The detailed derivations can be found in Appendix A.2.

Furthermore, with the maximum principle, when $f \in C(\bar{\Omega})$ and $u_{\delta}=u_{0}$ continuous in $\partial \Omega_{D \delta}$, we have the following lemma

Lemma 4.3. Suppose that a nonnegative continuous function $\phi(\mathbf{x})$ is defined on $\bar{\Omega} \cup \partial \Omega_{D \delta}$, and $-L_{\delta} \phi \geq G(\mathbf{x})>$ 0 for $\mathbf{x} \in \Omega \backslash \Omega_{N \delta},-L_{N \delta} \phi \geq G(\mathbf{x})>0$ for $\mathbf{x} \in \Omega_{N \delta}$. Then

$$
\sup _{\mathbf{x} \in \Omega \cup \partial \Omega_{N}}\left|e_{\delta}(\mathbf{x})\right| \leq \sup _{\mathbf{x} \in \partial \Omega_{D \delta}} \phi(\mathbf{x}) \sup _{\mathbf{x} \in \Omega \cup \partial \Omega_{N}} \frac{\left|T_{\delta}(\mathbf{x})\right|}{G(\mathbf{x})} .
$$

Proof. Let $K_{\delta}=\sup _{\mathbf{x} \in \Omega \cup \partial \Omega_{N}} \frac{\left|T_{\delta}(\mathbf{x})\right|}{G(\mathbf{x})}$, then for $K_{\delta} \phi(\mathbf{x})+e_{\delta}(\mathbf{x})$ we have: For $\mathbf{x} \in \Omega \backslash \Omega_{N \delta}$

$$
L_{\delta}\left(K_{\delta} \phi(\mathbf{x})+e_{\delta}(\mathbf{x})\right)=\sup _{\mathbf{x} \in \Omega \cup \partial \Omega_{N}} \frac{\left|T_{\delta}(\mathbf{x})\right|}{G(\mathbf{x})} L_{\delta} \phi(\mathbf{x})+L_{\delta} e_{\delta}(\mathbf{x})=\sup _{\mathbf{x} \in \Omega \cup \partial \Omega_{N}} \frac{\left|T_{\delta}(\mathbf{x})\right|}{G(\mathbf{x})} L_{\delta} \phi(\mathbf{x})+T_{\delta} \leq 0
$$

and a similar argument holds for $\mathbf{x} \in \Omega_{N \delta}$. With the maximum principle in Lemma 4.1 we have

$$
\sup _{\mathbf{x} \in \Omega \cup \partial \Omega_{N}} e_{\delta}(\mathbf{x}) \leq \sup _{\mathbf{x} \in \Omega \cup \partial \Omega_{N}}\left(K_{\delta} \phi(\mathbf{x})+e_{\delta}(\mathbf{x})\right) \leq \sup _{\mathbf{x} \in \partial \Omega_{D \delta}}\left(K_{\delta} \phi(\mathbf{x})+e_{\delta}(\mathbf{x})\right)=K_{\delta} \sup _{\mathbf{x} \in \partial \Omega_{D \delta}} \phi(\mathbf{x}) .
$$

Similarly, we have $L_{\delta}\left(K_{\delta} \phi(\mathbf{x})-e_{\delta}(\mathbf{x})\right) \leq 0$ for $\mathbf{x} \in \Omega \backslash \Omega_{N \delta}$ and $L_{N \delta}\left(K_{\delta} \phi(\mathbf{x})-e_{\delta}(\mathbf{x})\right) \leq 0$ for $\mathbf{x} \in \Omega_{N \delta}$, hence

$$
\sup _{\mathbf{x} \in \Omega \cup \partial \Omega_{N}}\left(-e_{\delta}(\mathbf{x})\right) \leq \sup _{\mathbf{x} \in \Omega \cup \partial \Omega_{N}}\left(K_{\delta} \phi(\mathbf{x})-e_{\delta}(\mathbf{x})\right) \leq \sup _{\mathbf{x} \in \partial \Omega_{D \delta}}\left(K_{\delta} \phi(\mathbf{x})-e_{\delta}(\mathbf{x})\right)=K_{\delta} \sup _{\mathbf{x} \in \partial \Omega_{D \delta}} \phi(\mathbf{x}) .
$$

We now define a nonnegative continuous function $\phi$ satisfying the conditions given in Lemma 4.3. In the following we take a specific kernel $J_{\delta}(\mathbf{s})=\frac{4}{\pi \delta^{4}}$ for $|\mathbf{s}| \leq \delta$ for simplicity. As shown in Figure 3, let $\left\{\mathbf{z}_{1}, \mathbf{z}_{2}\right\}:=$ $\partial \Omega_{D} \cap \partial \Omega_{N}$ and $\pi_{\partial \Omega}$ be the projection operator onto $\partial \Omega$. Due to the convexity of $\Omega$, the map $\pi_{\partial \Omega}(\mathbf{x})$ is always well defined and single-valued for any point $\mathbf{x} \notin \Omega$. For $\mathbf{x} \in \Omega$, the set where $\pi_{\partial \Omega}(\mathbf{x})$ is not single-valued (i.e., 
the "ridge" of $\partial \Omega)$ is $L^{2}$-negligible [49]. We then make the following crucial geometric assumption: Let $\tau\left(\mathbf{z}_{1}\right)$ (resp. $\tau\left(\mathbf{z}_{2}\right)$ ) be the tangent line to $\partial \Omega$ at $\mathbf{z}_{1}$ (resp. $\mathbf{z}_{2}$ ), then the intersecting point $\tilde{\mathbf{z}}:=\tau\left(\mathbf{z}_{1}\right) \cap \tau\left(\mathbf{z}_{2}\right)$ satisfies

$$
\pi_{\partial \Omega}(\tilde{\mathbf{z}}) \in \partial \Omega_{N} .
$$

Let $\mathbf{z}_{3} \in \partial \Omega$ be a point such that $\tau\left(\mathbf{z}_{3}\right)$ is orthogonal to the bisector of the angle $\angle \mathbf{z}_{2} \tilde{\mathbf{z}} \mathbf{z}_{1}$. Set the barrier function as

$$
\phi(\mathbf{x}):=\left|\operatorname{dist}\left(\mathbf{x}, \tau\left(\mathbf{z}_{3}\right)\right)+1\right|^{2} .
$$

For any point $\overline{\mathbf{x}} \in \partial \Omega_{N}$, in the following we denote the angle between $\mathbf{p}(\overline{\mathbf{x}})$ and $\mathbf{n}\left(\mathbf{z}_{3}\right)$ as $\alpha(\overline{\mathbf{x}})$. Note that with the crucial geometric assumption and the fact the $\Omega$ is convex, there exists $0<\tilde{\alpha}<\pi / 2$ such that $\tilde{\alpha} \leq \alpha(\overline{\mathbf{x}}) \leq \pi-\tilde{\alpha}, \forall \overline{\mathbf{x}} \in \partial \Omega_{N}$. Let $\Pi$ be the half-plane delimited by $\tau\left(\mathbf{z}_{3}\right)$ and containing $\Omega$, we now check the conditions in Lemma 4.3 with the following 3 steps:

Step 1: Convexity of $\phi$. To check that $\phi$ is convex on $\Pi$, consider arbitrary points $\mathbf{x}, \mathbf{y} \in \Pi$, and $t \in(0,1)$. We need to check

$$
\phi((1-t) \mathbf{x}+t \mathbf{y}) \leq(1-t) \phi(\mathbf{x})+t \phi(\mathbf{y}) .
$$

By construction, $\phi$ is invariant in the direction of $\tau\left(\mathbf{z}_{3}\right)$. Letting $\Sigma$ be an arbitrary line orthogonal to $\tau\left(\mathbf{z}_{3}\right)$ and $\mathbf{x}^{*}$ (resp. $\left.\mathbf{y}^{*}\right)$ be the projections of $\mathbf{x}$ (resp. $\left.\mathbf{y}\right)$ on $\Sigma$ for the projection of $(1-t) \mathbf{x}+t \mathbf{y}$ on $\Sigma$, we get

$$
[(1-t) \mathbf{x}+t \mathbf{y}]^{*}=(1-t) \mathbf{x}^{*}+t \mathbf{y}^{*}
$$

Since $\phi$ is invariant in the direction of $\tau\left(\mathbf{z}_{3}\right)$, we get

$$
\phi((1-t) \mathbf{x}+t \mathbf{y})=\phi\left((1-t) \mathbf{x}^{*}+t \mathbf{y}^{*}\right), \quad \phi(\mathbf{x})=\phi\left(\mathbf{x}^{*}\right), \quad \phi(\mathbf{y})=\phi\left(\mathbf{y}^{*}\right),
$$

and (4.16) is equivalent to

$$
\phi\left((1-t) \mathbf{x}^{*}+t \mathbf{y}^{*}\right) \leq(1-t) \phi\left(\mathbf{x}^{*}\right)+t \phi\left(\mathbf{y}^{*}\right) .
$$

Note that (4.17) holds true due to the convexity of $\phi$ along the direction $\mathbf{n}\left(\mathbf{z}_{3}\right) \| \Sigma$. The convexity of $\phi$ gives $[\phi]_{v v} \geq 0$ for any (nonzero) vector $\mathbf{v}$. Combining with the facts $0 \leq M_{\delta}(\mathbf{x}) \leq C$ as shown in Lemma 3.1 and $\int_{-\delta}^{\delta} H(|l|)\left[\phi\left(\mathbf{x}_{l}\right)-\phi(\mathbf{x})\right] \mathrm{d} \mathbf{x}_{l}=\frac{1}{2}[\phi]_{p p}+\frac{\kappa}{2}[\phi]_{n n}(\mathbf{x}-\overline{\mathbf{x}}) \cdot \mathbf{x}$ as shown in (A.7), we infer directly that

$$
M_{\delta}(\mathbf{x}) \int_{-\delta}^{\delta} H(|l|)\left[\phi\left(\mathbf{x}_{l}\right)-\phi(\mathbf{x})\right] \mathrm{d} \mathbf{x}_{l} \geq 0 .
$$

It remains to show the bounds for

$$
\begin{array}{cc}
\int_{\left(\Omega \cup \partial \Omega_{D \delta}\right) \cap B(\mathbf{x}, \delta)}(\phi(\mathbf{y})-\phi(\mathbf{x})) \mathrm{d} \mathbf{y}, \quad & \forall \mathbf{x} \in \Omega \backslash \Omega_{N \delta}, \\
\int_{\Omega \cap B(\mathbf{x}, \delta)}(\phi(\mathbf{y})-\phi(\mathbf{x})) \mathrm{d} \mathbf{y}, & \forall \mathbf{x} \in \Omega_{N \delta} .
\end{array}
$$

Step 2: bound for (4.19). Note that in this case $B(\mathbf{x}, \delta) \subset \Omega \cup \partial \Omega_{D \delta}$. Let $\ell(\mathbf{x})$ be the line through $\mathbf{x}$ and parallel to $\ell:=\tau\left(\mathbf{z}_{3}\right)$. Noting that $B(\mathbf{x}, \delta)$ is symmetric with respect to $\ell(\mathbf{x})$, for any $\mathbf{y} \in B(\mathbf{x}, \delta)$ we denote by $\mathbf{y}^{*}$ the reflection of $\mathbf{y}$ across $\ell(\mathbf{x})$. Let $B^{+}(\mathbf{x}, \delta)$ (resp. $B^{-}(\mathbf{x}, \delta)$ ) be the "upper" (resp. "lower") half ball, then

$$
\begin{aligned}
\int_{\left(\Omega \cup \partial \Omega_{D \delta}\right) \cap B(\mathbf{x}, \delta)}(\phi(\mathbf{y})-\phi(\mathbf{x})) \mathrm{d} \mathbf{y} & =\int_{B^{+}(\mathbf{x}, \delta)}(\phi(\mathbf{y})-\phi(\mathbf{x}))+\left(\phi\left(\mathbf{y}^{*}\right)-\phi(\mathbf{x})\right) \mathrm{d} \mathbf{y} \\
& \left.=\int_{0}^{\delta}\left[(\sqrt{\phi(\mathbf{x})}+\rho)^{2}+(\sqrt{\phi(\mathbf{x})}-\rho)^{2}-2 \phi(\mathbf{x})\right)\right] 2 \sqrt{\delta^{2}-\rho^{2}} \mathrm{~d} \rho \\
& =4 \int_{0}^{\delta} \rho^{2} \sqrt{\delta^{2}-\rho^{2}} \mathrm{~d} \rho \\
& \geq 4 \int_{0}^{\delta / 2} \rho^{2} \sqrt{\delta^{2}-\delta^{2} / 4} \mathrm{~d} \rho=\frac{\delta^{4}}{4 \sqrt{3}} .
\end{aligned}
$$




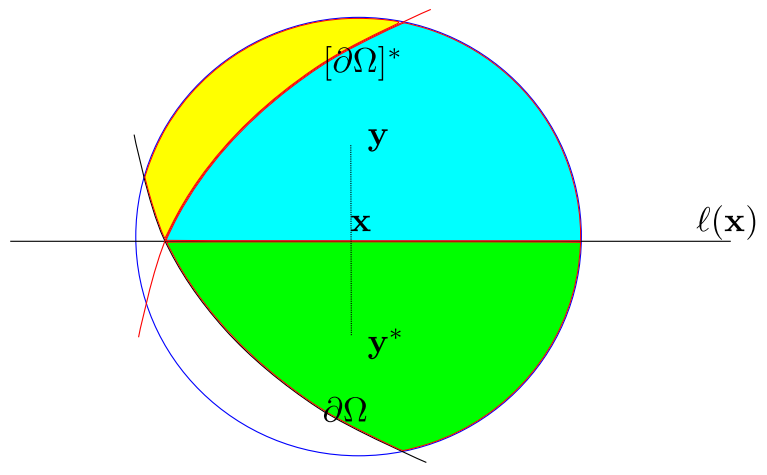

FIgURE 4. Notation for estimating the bound of $\int_{\Omega \cap B(\mathbf{x}, \delta)}(\phi(\mathbf{y})-\phi(\mathbf{x}))$ dy when $\mathbf{x} \in \Omega_{N \delta}$. Here green denotes the region of $B^{-}(\mathbf{x}, \delta) \cap \Omega$, cyan denotes $\left[B^{-}(\mathbf{x}, \delta) \cap \Omega\right]^{*}$, and yellow denotes $\left[\Omega \cap B^{+}(\mathbf{x}, \delta)\right] \backslash\left[B^{-}(\mathbf{x}, \delta) \cap \Omega\right]^{*} .[\partial \Omega]^{*}$ is the reflection of $\partial \Omega$ across $\ell(\mathbf{x})$.

Recalling $J_{\delta}=\frac{4}{\pi \delta^{4}}$ on its support, we obtained $-L_{\delta} \phi(\mathbf{x}) \geq \frac{2}{\pi \sqrt{3}}, \forall \mathbf{x} \in \Omega \backslash \Omega_{N \delta}$.

Step 3: bound of (4.20). For $\mathbf{x} \in \Omega_{N \delta}$, we will show that

$$
\int_{\Omega \cap(\mathbf{x}, \delta)}(\phi(\mathbf{y})-\phi(\mathbf{x})) \mathbf{y} \geq \begin{cases}\delta^{3}, & \text { when } s_{x} \leq \delta / 2, \\ \delta^{4}+C\left(\delta-s_{x}\right)^{3 / 2} \delta^{3 / 2}, & \text { when } s_{x}>\delta / 2 .\end{cases}
$$

Let $\left[B^{-}(\mathbf{x}, \delta) \cap \Omega\right]^{*}$ be the reflection of $B^{-}(\mathbf{x}, \delta) \cap \Omega$ across $\ell(\mathbf{x})$, as shown in Figure 4. Note the crucial geometric condition ensures that $\left[B^{-}(\mathbf{x}, \delta) \cap \Omega\right]^{*} \subseteq B^{+}(\mathbf{x}, \delta) \cap \Omega$. Since

$$
(\phi(\mathbf{y})-\phi(\mathbf{x}))+\left(\phi\left(\mathbf{y}^{*}\right)-\phi(\mathbf{x})\right)=2 \operatorname{dist}(\mathbf{y}, \ell(\mathbf{x}))^{2},
$$

we have

$$
\begin{aligned}
\int_{\Omega \cap B(\mathbf{x}, \delta)}(\phi(\mathbf{y})-\phi(\mathbf{x})) \mathrm{d} \mathbf{y}= & \int_{\left[\Omega \cap B^{+}(\mathbf{x}, \delta)\right] \backslash\left[B^{-}(\mathbf{x}, \delta) \cap \Omega\right]^{*}}(\phi(\mathbf{y})-\phi(\mathbf{x})) \mathrm{d} \mathbf{y} \\
& +\int_{\left[B^{-}(\mathbf{x}, \delta) \cap \Omega\right]^{*}}(\phi(\mathbf{y})-\phi(\mathbf{x})) \mathrm{d} \mathbf{y}+\int_{\left[B^{-}(\mathbf{x}, \delta) \cap \Omega\right]^{*}}\left(\phi\left(\mathbf{y}^{*}\right)-\phi(\mathbf{x})\right) \mathrm{d} \mathbf{y} \\
\geq & \int_{\left[B^{-}(\mathbf{x}, \delta) \cap \Omega\right]^{*}}\left[(\phi(\mathbf{y})-\phi(\mathbf{x}))+\left(\phi\left(\mathbf{y}^{*}\right)-\phi(\mathbf{x})\right)\right] \mathrm{d} \mathbf{y} \geq 0 .
\end{aligned}
$$

Since $\mathbf{x} \in \Omega$, one has $\left|B^{-}(\mathbf{x}, \delta) \cap \Omega\right|>0$ unless $\mathbf{x} \in \partial \Omega$ and $\left(\partial \Omega \cap B^{-}(\mathbf{x}, \delta)\right) \subset\left(\ell(\mathbf{x}) \cap B^{-}(\mathbf{x}, \delta)\right)$. Therefore, using (4.21), when $s_{x}>\delta / 2$ and $\delta \leq D / 5 \leq\left(5 \sup _{\mathbf{z} \in \partial \Omega}|\kappa(\mathbf{z})|\right)^{-1}$, a direct computation gives

$$
\begin{aligned}
& \int_{\left[B^{-}(\mathbf{x}, \delta) \cap \Omega\right]^{*}}(\phi(\mathbf{y})-\phi(\mathbf{x})) \mathrm{d} \mathbf{y}+\int_{\left[B^{-}(\mathbf{x}, \delta) \cap \Omega\right]^{*}}\left(\phi\left(\mathbf{y}^{*}\right)-\phi(\mathbf{x})\right) \mathrm{d} \mathbf{y} \\
& \quad \geq \int_{0}^{\delta / 2-\delta / 12} 2 \rho^{2} \sqrt{\delta^{2}-\rho^{2}} \mathrm{~d} \rho \geq \int_{0}^{5 \delta / 12} 2 \rho^{2} \sqrt{\delta^{2}-(\delta / 2)^{2}} \mathrm{~d} \rho \geq \frac{125 \sqrt{3} \delta^{4}}{5184} .
\end{aligned}
$$

On the other hand, when $s_{x}>\delta / 2$ we calculate the integral on the purple region (denoted as $F$ ) shown in the left plot of Figure 5. With the geometric assumption, we have $\phi\left(\mathbf{y}_{B}\right)-\phi(\mathbf{x}) \geq 2 \operatorname{dist}\left(\mathbf{y}_{B}, l(\mathbf{x})\right) \geq 2 s_{x} \sin (\tilde{\alpha})>$ $\sin (\tilde{\alpha}) \delta$, where $\mathbf{y}_{B}$ denotes the coordinate of point $B$. Since $\int_{\left[\Omega \cap B^{+}(\mathbf{x}, \delta)\right] \backslash\left[B^{-}(\mathbf{x}, \delta) \cap \Omega\right]^{*}}(\phi(\mathbf{y})-\phi(\mathbf{x})) \mathrm{d} \mathbf{y} \geq \int_{F}(\phi(\mathbf{y})-$ $\phi(\mathbf{x}))$ dy and $|C D|=\sqrt{\delta^{2}-s_{x}^{2}} / 2,|C E| \geq\left(\delta-s_{x}\right) / 2$ when $\delta \ll 1 / \sup _{\mathbf{z} \in \partial \Omega}|\kappa(\mathbf{z})|$, for $\mathbf{y} \in F$ we have

$$
\phi(\mathbf{y})-\phi(\mathbf{x}) \geq \phi\left(\mathbf{y}_{B}\right)-\phi(\mathbf{x}) \geq \sin (\tilde{\alpha}) \delta,
$$




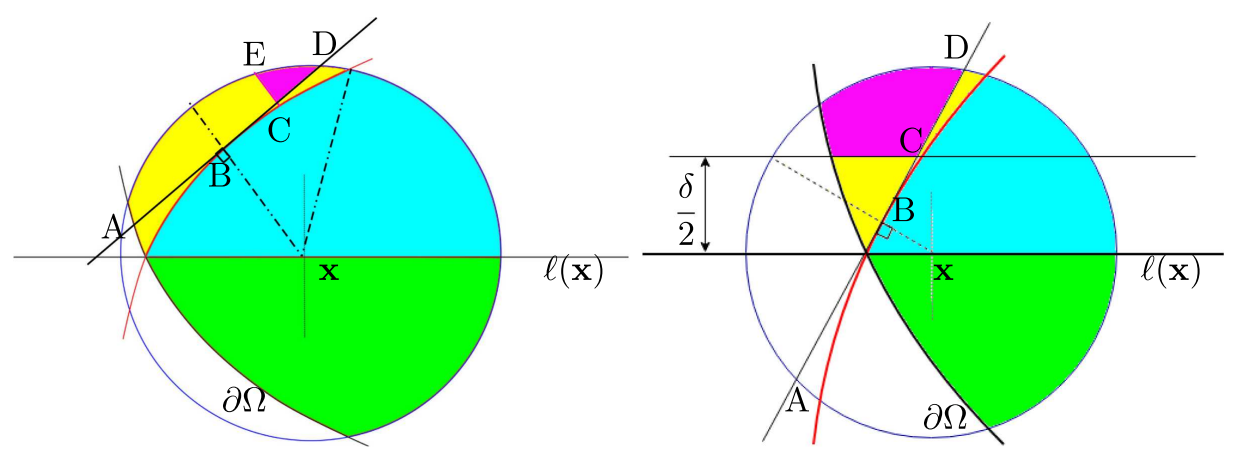

Figure 5. Notation for estimating the bound of $\int_{\Omega \cap B(\mathbf{x}, \delta)}(\phi(\mathbf{y})-\phi(\mathbf{x})) \mathrm{d} \mathbf{y}$ when $\mathbf{x} \in \Omega_{N \delta}$, where the green and cyan regions denote $B^{-}(\mathbf{x}, \delta) \cap \Omega$ and $\left[B^{-}(\mathbf{x}, \delta) \cap \Omega\right]^{*}$, respectively. The union of yellow and purple regions represent $\left[\Omega \cap B^{+}(\mathbf{x}, \delta)\right] \backslash\left[B^{-}(\mathbf{x}, \delta) \cap \Omega\right]^{*}$. Left: notation when $\operatorname{dist}(\mathbf{x}, \partial \Omega)>\delta / 2$, where the purple region is chosen such that $\mathbf{p}\left(\mathbf{y}_{B}\right) \perp x B, \mathbf{n}\left(\mathbf{y}_{B}\right) \| C E$ and $|B C|=|B D|$. Right: notation when $\operatorname{dist}(\mathbf{x}, \partial \Omega) \leq \delta / 2$, where the purple region is chosen such that $\mathbf{p}\left(\mathbf{y}_{B}\right) \perp x B$ and the distance from $C$ to $l(\mathbf{x})$ is $\delta / 2$.

and

$$
\operatorname{area}(F)>\operatorname{area}\left(\triangle_{C D E}\right)=\frac{1}{8} \sqrt{\left|\delta-s_{x}\right|^{3}\left(\delta+s_{x}\right)} \geq \frac{1}{8} \delta^{1 / 2}\left(\delta-s_{x}\right)^{3 / 2} .
$$

We then have

$$
\int_{\left[\Omega \cap B^{+}(\mathbf{x}, \delta)\right] \backslash\left[B^{-}(\mathbf{x}, \delta) \cap \Omega\right]^{*}}(\phi(\mathbf{y})-\phi(\mathbf{x})) \mathrm{d} \mathbf{y} \geq C\left(\delta-s_{x}\right)^{3 / 2} \delta^{3 / 2} .
$$

Similarly, for $s_{x} \leq \delta / 2$ we have $G \subset\left[\Omega \cap B^{+}(\mathbf{x}, \delta)\right] \backslash\left[B^{-}(\mathbf{x}, \delta) \cap \Omega\right]^{*}$ where $G$ is the purple set denoted in the right plot of Figure 5. For $\mathbf{y} \in G$ we have $\phi(\mathbf{y})-\phi(\mathbf{x}) \geq \delta$ and $\operatorname{area}(G) \geq \min \left\{\frac{(\sqrt{3}-1)^{2} \delta^{2} \tan (\tilde{\alpha})}{8}, \frac{\sqrt{3}}{8} \delta^{2}\right\}=C \delta^{2}$. Therefore

$$
\int_{\left[\Omega \cap B^{+}(\mathbf{x}, \delta)\right] \backslash\left[B^{-}(\mathbf{x}, \delta) \cap \Omega\right]^{*}}(\phi(\mathbf{y})-\phi(\mathbf{x})) \mathrm{d} \mathbf{y} \geq C \delta^{3} .
$$

i.e., the contribution of a region that lies completely above $\ell(\mathbf{x})$ is of order $O\left(\delta^{3}\right)$, provided that it has positive area.

Thus (4.19) and (4.20) are bounded. Combining with (4.18), and recalling $J_{\delta}=A \delta^{-4}$ on its support, we get

$$
-L_{\delta} \phi(\mathbf{x})=2 \int_{\Omega \cup \partial \Omega_{D \delta}} J_{\delta}(|\mathbf{x}-\mathbf{y}|)(\phi(\mathbf{y})-\phi(\mathbf{x})) \mathrm{d} \mathbf{y} \geq C
$$

for all $\mathbf{x} \in \Omega \backslash \Omega_{N \delta}$, and

$$
\begin{aligned}
-L_{N \delta} \phi(\mathbf{x}) & =2 \int_{\Omega} J_{\delta}(|\mathbf{x}-\mathbf{y}|)(\phi(\mathbf{y})-\phi(\mathbf{x})) \mathrm{d} \mathbf{y}+2 M_{\delta}(\mathbf{x}) \int_{-\delta}^{\delta} H(|l|)\left[u_{0}\left(\mathbf{x}_{l}\right)-u_{0}(\mathbf{x})\right] \mathrm{d} \mathbf{x}_{l} \\
& \geq C\left[\delta-s_{x}\right]^{3 / 2} \delta^{-5 / 2}+C_{1}>0
\end{aligned}
$$

for all $\mathbf{x} \in \Omega_{N \delta}$.

Note that Lemma 4.2 and the above estimates on function $\phi$ are still insufficient to ensure second order $L^{\infty}(\Omega)$ convergence to the local limit, since Lemma 4.2 gives $T_{\delta}=O(\delta)$ on $\Omega_{N \delta}$, while the estimates for $\phi$ gives only

$$
-L_{N \delta} \phi \geq C\left[\delta-s_{x}\right]^{3 / 2} \delta^{-5 / 2}+C_{1},
$$


and it is unclear if $\frac{T_{\delta}}{-L_{N \delta} \phi}$ can be uniformly bounded from above by $C \delta^{2}$ as $\mathbf{x}$ approaches the inner boundary of $\Omega_{N \delta}$. The next Lemma aims to provide an estimate for $T_{\delta}$.

Lemma 4.4. For $\mathbf{x} \in \Omega_{N \delta}, T_{\delta}(\mathbf{x})$ is bounded by $C \delta$ when $\mathbf{x}$ is close to $\partial \Omega$ and bounded by $C \delta^{2}$ as $\mathbf{x}$ approaches the inner boundary of $\Omega_{N \delta}$. Specifically,

$$
\left|T_{\delta}\right| \leq C\left[\delta-s_{x}\right]^{3 / 2} \delta^{-1 / 2}+O\left(\delta^{2}\right) .
$$

Proof. By Lemma 4.2 and the facts $\int_{B(\mathbf{x}, \delta)} J_{\delta}(|\mathbf{x}-\mathbf{y}|)((\mathbf{x}-\mathbf{y}) \cdot \mathbf{n}(\overline{\mathbf{x}}))^{3} \mathrm{~d} \mathbf{y}=0, \int_{B(\mathbf{x}, \delta)} J_{\delta}(|\mathbf{x}-\mathbf{y}|)((\mathbf{x}-\mathbf{y}) \cdot \mathbf{n}(\overline{\mathbf{x}})) \mathrm{d} \mathbf{y}=$ 0 and $\int_{B(\mathbf{x}, \delta)} J_{\delta}(|\mathbf{x}-\mathbf{y}|)((\mathbf{x}-\mathbf{y}) \cdot \mathbf{n}(\overline{\mathbf{x}}))|(\mathbf{x}-\mathbf{y}) \cdot \mathbf{p}(\overline{\mathbf{x}})|^{2} \mathrm{~d} \mathbf{y}=0$, we have

$$
\begin{aligned}
T_{\delta}= & 2 \int_{E_{\delta}} J_{\delta}(|\mathbf{x}-\mathbf{y}|) \frac{\partial u_{0}(\mathbf{x})}{\partial \mathbf{p}}((\mathbf{x}-\mathbf{y}) \cdot \mathbf{p}(\overline{\mathbf{x}})) \mathrm{d} \mathbf{y} \\
& -\left[u_{0}(\mathbf{x})\right]_{n n n}\left(\frac{1}{3} \int_{\partial \Omega_{N \delta}} J_{\delta}(|\mathbf{x}-\mathbf{y}|)((\mathbf{x}-\mathbf{y}) \cdot \mathbf{n}(\overline{\mathbf{x}}))^{3} \mathrm{~d} \mathbf{y}\right. \\
& \left.-\int_{\partial \Omega_{N \delta}} J_{\delta}(|\mathbf{x}-\mathbf{y}|)((\mathbf{x}-\mathbf{y}) \cdot \mathbf{n}(\overline{\mathbf{x}})) \mathrm{d} \mathbf{y}|\overline{\mathbf{x}}-\mathbf{x}|^{2}\right) \\
& -\int_{\partial \Omega_{N \delta}} J_{\delta}(|\mathbf{x}-\mathbf{y}|)\left[u_{0}(\mathbf{x})\right]_{n p p}((\mathbf{x}-\mathbf{y}) \cdot \mathbf{n}(\overline{\mathbf{x}}))|(\mathbf{x}-\mathbf{y}) \cdot \mathbf{p}(\overline{\mathbf{x}})|^{2} \mathrm{~d} \mathbf{y} \\
& +\kappa(\overline{\mathbf{x}})\left[u_{0}(\mathbf{x})\right]_{n n}((\mathbf{x}-\overline{\mathbf{x}}) \cdot \mathbf{n}(\overline{\mathbf{x}})) \int_{\partial \Omega_{N \delta}} J_{\delta}(|\mathbf{x}-\mathbf{y}|)\left(|(\mathbf{y}-\mathbf{x}) \cdot \mathbf{p}(\overline{\mathbf{x}})|^{2}\right. \\
& \left.-|(\mathbf{y}-\overline{\mathbf{x}}) \cdot \mathbf{n}(\overline{\mathbf{x}})|^{2}+|(\mathbf{x}-\overline{\mathbf{x}}) \cdot \mathbf{n}(\overline{\mathbf{x}})|^{2}\right) \mathrm{d} \mathbf{y}+O\left(\delta^{2}\right) .
\end{aligned}
$$

We firstly provide the bounds for the first term. Note that $|B(\mathbf{x}, \delta) \cap \partial \Omega| \leq 2 \sqrt{\delta^{2}-s_{x}^{2}}+C \kappa(\overline{\mathbf{x}})\left(\delta^{2}-s_{x}^{2}\right)$, therefore $|(\mathbf{x}-\mathbf{y}) \cdot \mathbf{p}(\overline{\mathbf{x}})| \leq C \sqrt{\delta^{2}-s_{x}^{2}}$ for $\mathbf{y} \in E_{\delta}$. Moreover, as shown in Appendix A.2, for the area of $E_{\delta}$ we have $\left|E_{\delta}\right| \leq C\left(\delta^{2}-s_{x}^{2}\right)^{2}+O\left(\delta^{5}\right)$. Then

$$
\left|\int_{E_{\delta}} J_{\delta}(|\mathbf{x}-\mathbf{y}|) \frac{\partial u_{0}(\mathbf{x})}{\partial \mathbf{p}}((\mathbf{x}-\mathbf{y}) \cdot \mathbf{p}(\overline{\mathbf{x}})) \mathrm{d} \mathbf{y}\right| \leq C\left(\delta^{2}-s_{x}^{2}\right)^{5 / 2} \delta^{-4} \leq C\left(\delta-s_{x}\right)^{3 / 2} \delta^{-1 / 2} .
$$

For the rest of terms in $T_{\delta}$, note that the integrands

$$
\begin{aligned}
& J_{\delta}(|\mathbf{x}-\mathbf{y}|)((\mathbf{x}-\mathbf{y}) \cdot \mathbf{n}(\overline{\mathbf{x}}))^{3} \leq C \delta^{-1} \\
& J_{\delta}(|\mathbf{x}-\mathbf{y}|)((\mathbf{x}-\mathbf{y}) \cdot \mathbf{n}(\overline{\mathbf{x}}))|\overline{\mathbf{x}}-\mathbf{x}|^{2} \leq C \delta^{-1} \\
& J_{\delta}(|\mathbf{x}-\mathbf{y}|)((\mathbf{x}-\mathbf{y}) \cdot \mathbf{n}(\overline{\mathbf{x}}))|(\mathbf{x}-\mathbf{y}) \cdot \mathbf{p}(\overline{\mathbf{x}})|^{2} \leq C \delta^{-1} \\
& J_{\delta}(|\mathbf{x}-\mathbf{y}|)|(\mathbf{y}-\mathbf{x}) \cdot \mathbf{p}(\overline{\mathbf{x}})|^{2}((\mathbf{x}-\overline{\mathbf{x}}) \cdot \mathbf{n}(\overline{\mathbf{x}})) \leq C \delta^{-1} \\
& J_{\delta}(|\mathbf{x}-\mathbf{y}|)|(\mathbf{y}-\overline{\mathbf{x}}) \cdot \mathbf{n}(\overline{\mathbf{x}})|^{2}((\mathbf{x}-\overline{\mathbf{x}}) \cdot \mathbf{n}(\overline{\mathbf{x}})) \leq C \delta^{-1} \\
& J_{\delta}(|\mathbf{x}-\mathbf{y}|)|(\mathbf{x}-\overline{\mathbf{x}}) \cdot \mathbf{n}(\overline{\mathbf{x}})|^{2}((\mathbf{x}-\overline{\mathbf{x}}) \cdot \mathbf{n}(\overline{\mathbf{x}})) \leq C \delta^{-1}
\end{aligned}
$$

for some constant $C$. Thus it suffices to estimate the area of the domain of integration $\partial \Omega_{N \delta} \cap B(\mathbf{x}, \delta)$. Since $\left|A_{\delta}\right| \leq C \delta^{3}$, it suffices the compute the area of $D_{\delta}$. Since $D_{\delta}$ is contained in the rectangle with side lengths $2 \sqrt{\delta^{2}-s_{x}^{2}}$ and $\delta-s_{x}$, direct computation then gives

$$
\left|D_{\delta}\right| \leq 2\left(\delta-s_{x}\right) \sqrt{\delta^{2}-s_{x}^{2}} \leq 2 \sqrt{2}\left[\delta-s_{x}\right]^{3 / 2} \delta^{1 / 2} .
$$

We then have $\left|\partial \Omega_{N \delta} \cap B(\mathbf{x}, \delta)\right| \leq C\left[\delta-s_{x}\right]^{3 / 2} \delta^{1 / 2}+C \delta^{3}$ which together with the bounds of the integrands finishes the proof. 
With the above lemmas we obtain the main theorem of this section.

Theorem 4.5. Suppose $f \in C(\bar{\Omega})$, $u_{\delta}$ solves the nonlocal problem (4.2) and $u_{0}$ is the solution to the corresponding local problem (4.3), then for sufficiently small $\delta$ there exists a constant $C$ independent of $\delta$ such that

$$
\sup _{\mathbf{x} \in \Omega}\left|u_{\delta}(\mathbf{x})-u_{0}(\mathbf{x})\right| \leq C \delta^{2} .
$$

Proof. With the barrier function $\phi$ defined as in (4.15), from the above lemmas and bounds we have

$$
\begin{array}{r}
\frac{\left|T_{\delta}\right|}{-L_{\delta} \phi} \leq C \delta^{2}, \quad \text { for } \mathbf{x} \in \Omega \backslash \Omega_{N \delta}, \\
\frac{\left|T_{\delta}\right|}{-L_{N \delta} \phi} \leq \frac{C_{1}\left[\delta-s_{x}\right]^{3 / 2} \delta^{-1 / 2}+C_{2} \delta^{2}}{C_{3}\left[\delta-s_{x}\right]^{3 / 2} \delta^{-5 / 2}+C_{4}}, \quad \text { for } \mathbf{x} \in \Omega_{N \delta} .
\end{array}
$$

Therefore, with Lemma 4.3, the proof of (4.22) will be finished once we can show that $\frac{\left|T_{\delta}\right|}{-L_{N \delta} \phi} \leq C \delta^{2}$ for $\mathbf{x} \in \Omega_{N \delta}$. Let

$$
f(r):=\frac{C_{1}[\delta-r]^{3 / 2} \delta^{-1 / 2}+C_{2} \delta^{2}}{C_{3}[\delta-r]^{3 / 2} \delta^{-5 / 2}+C_{4}}, \quad r:=s_{x} \in[0, \delta]
$$

Then

$$
f^{\prime}(r)=\left(\frac{3}{2}\right) \frac{\left(C_{2} C_{3}-C_{1} C_{4}\right)[\delta-r]^{1 / 2} \delta^{-1 / 2}}{\left[C_{3}[\delta-r]^{3 / 2} \delta^{-5 / 2}+C_{4}\right]^{2}},
$$

and thus $f$ is monotone (either increasing or decreasing, depending on the sign of $C_{2} C_{3}-C_{1} C_{4}$ ). Since

$$
f(0)=\frac{C_{1} \delta+C_{2} \delta^{2}}{C_{3} \delta^{-1}+C_{4}}=\frac{C_{1} \delta^{2}+C_{2} \delta^{3}}{C_{3}+C_{4} \delta} \leq O\left(\delta^{2}\right), \quad f(\delta)=\frac{C_{2} \delta^{2}}{C_{4}},
$$

the monotonicity of $f$ ensures that $f \leq O\left(\delta^{2}\right)$ for all $r \in[0, \delta]$, hence we get

$$
\sup _{\mathbf{x} \in \Omega_{N \delta}} \frac{\left|T_{\delta}\right|}{-L_{N \delta} \phi} \leq O\left(\delta^{2}\right)
$$

\section{Meshfree QuAdrature Rule AND Numerical SOlVER}

In this section, we develop a discretization method based upon a meshfree quadrature rule for compactly supported nonlocal integro-differential equations (IDEs) with radial kernels. This approach is based upon the generalized moving least squares (GMLS) approximation framework [64], and falls within the scope of the well-established GMLS approximation theory.

We discretize the domain $\Omega$ and $\partial \Omega_{D \delta}$ by a collection of points $\chi_{h}=\left\{\mathbf{x}_{i}\right\}_{\left\{i=1,2, \cdots, N_{p}\right\}} \subset \Omega \cup \partial \Omega_{D \delta}$, where the fill distance

$$
h:=\sup _{\mathbf{x}_{i} \in \chi_{h}} \min _{1 \leq j \leq N_{p}, j \neq i}\left|\mathbf{x}_{i}-\mathbf{x}_{j}\right|
$$

is a length scale characterizing the resolution of the point cloud, and $N_{p}$ denotes the total number of points. We define the separation distance

$$
q_{\chi}=\frac{1}{2} \min _{i \neq j}\left|x_{i}-x_{j}\right|
$$


and assume that the point set is quasi-uniform, namely that there exists a positive $c_{q u}$ satisfying

$$
q_{\chi} \leq h \leq c_{q u} q_{\chi}
$$

In a neighborhood of each point $\mathbf{x}_{i} \in \chi_{h}$, we reconstruct a polynomial approximation $s_{u, \chi_{h}, i}(\mathbf{x})$ to the nonlocal solution $u_{\delta}(\mathbf{x})$ in $B\left(\mathbf{x}_{i}, \delta\right)$. Specifically, we define $s_{u, \chi_{h}, i}$ as the solution to the optimization problem

$$
s_{u, \chi_{h}, i}(\mathbf{x})=\min _{p \in \pi_{m}\left(\mathbb{R}^{2}\right)}\left\{\sum_{j=1}^{N_{p}}\left[u\left(\mathbf{x}_{j}\right)-p\left(\mathbf{x}_{j}\right)\right]^{2} w\left(\mathbf{x}_{i}, \mathbf{x}_{j}\right)\right\},
$$

where $\pi_{m}\left(\mathbb{R}^{2}\right)$ are the $m$-th order polynomials in $\mathbb{R}^{2}$, and $w(\mathbf{x}, \mathbf{y})$ is a translation-invariant positive weight function with compact support $\delta$. For concreteness we take in this work

$$
w(\mathbf{x}, \mathbf{y})=\Phi_{\delta}(\mathbf{x}-\mathbf{y})=\left\{\begin{array}{cc}
\left(1-\frac{|\mathbf{x}-\mathbf{y}|}{\delta}\right)^{4}, & \text { when }|\mathbf{x}-\mathbf{y}| \leq \delta \\
0 & \text { when }|\mathbf{x}-\mathbf{y}|>\delta
\end{array}\right.
$$

For a quasi-uniform pointset and sufficiently large $\delta$ the optimization problem possesses a unique solution [66]. We then use this polynomial reconstruction to approximate the nonlocal operator as follows.

For each point $\mathbf{x}_{i}$, denote the set of indices for points in $B\left(\mathbf{x}_{i}, \delta\right)$ as

$$
I\left(\mathbf{x}_{i}\right) \equiv I\left(\mathbf{x}_{i}, \delta, \chi_{h}\right):=\left\{j \in\left\{1, \cdots, N_{p}\right\}:\left|\mathbf{x}_{i}-\mathbf{x}_{j}\right|<\delta\right\},
$$

and $\# I\left(\mathbf{x}_{i}\right)$ represents the number of indices in $I\left(\mathbf{x}_{i}\right)$. Define as a basis for $\pi_{m}\left(\mathbb{R}^{2}\right)$ the set $p_{1}(\mathbf{x}), p_{2}(\mathbf{x}), \cdots, p_{Q}(\mathbf{x})$, then the optimization problem has the following analytic solution.

$$
s_{u, \chi_{h}, i}(\mathbf{x})=\tilde{u} D P\left(P^{\mathrm{T}} D P\right)^{-1} R(\mathbf{x}),
$$

where

$$
\begin{aligned}
\tilde{u} & :=\left(u\left(\mathbf{x}_{j}\right): j \in I\left(\mathbf{x}_{i}\right)\right)^{\mathrm{T}} \in \mathbb{R}^{\# I\left(\mathbf{x}_{i}\right)}, \\
P & :=\left(p_{k}\left(\mathbf{x}_{j}\right)\right)_{j \in I\left(\mathbf{x}_{i}\right), 1 \leq k \leq Q} \in \mathbb{R}^{\# I\left(\mathbf{x}_{i}\right) \times Q} \\
D & =\operatorname{diag}\left(\Phi_{\delta}\left(\mathbf{x}_{i}-\mathbf{x}_{j}\right): j \in I\left(\mathbf{x}_{i}\right)\right) \in \mathbb{R}^{\# I\left(\mathbf{x}_{i}\right) \times \# I\left(\mathbf{x}_{i}\right)}, \\
R(\mathbf{x}) & =\left(p_{1}(\mathbf{x}), \cdots, p_{Q}(\mathbf{x})\right)^{\mathrm{T}} \in \mathbb{R}^{Q} .
\end{aligned}
$$

This process exactly recovers $u \in \pi_{m}\left(\mathbb{R}^{2}\right)$. In the GMLS framework, the reconstruction may be used to approximate a linear bounded target functional $\varpi$ as

$$
\varpi(u) \approx \varpi_{h}(u):=\varpi\left(s_{u, \chi_{h}, i}\right)=\tilde{u} D P\left(P^{\mathrm{T}} D P\right)^{-1} \varpi(R(\mathbf{x})),
$$

where $\varpi(R)$ denotes the application of the target functional component-wise to each element of the polynomial basis. Classic examples of $\varpi$ include the point evaluation functional to develop meshfree approximants, point evaluations of derivatives of functions to develop meshfree collocation schemes, and integrals of functions over compact sets. In this work, we select $\varpi$ as the nonlocal operator in (1.1) and (2.5), and thus obtain a meshfree estimator of the non-local operator that is exact when applied to $\pi_{m}\left(\mathbb{R}^{2}\right)$. To do so will require the computation of (1.1) and (2.5) applied to each member of the polynomial space.

In this paper we take $m=2$ and choose the quadratic basis functions as follows

$$
\begin{aligned}
& p_{1}(\mathbf{x})=1, \quad p_{2}(\mathbf{x})=\left(\mathbf{x}-\mathbf{x}_{i}\right) \cdot \mathbf{e}_{1}, \quad p_{3}(\mathbf{x})=\left(\mathbf{x}-\mathbf{x}_{i}\right) \cdot \mathbf{e}_{2}, \quad p_{4}(\mathbf{x})=\left[\left(\mathbf{x}-\mathbf{x}_{i}\right) \cdot \mathbf{e}_{1}\right]^{2}, \\
& p_{5}(\mathbf{x})=\left[\left(\mathbf{x}-\mathbf{x}_{i}\right) \cdot \mathbf{e}_{2}\right]^{2}, \quad p_{6}(\mathbf{x})=\left[\left(\mathbf{x}-\mathbf{x}_{i}\right) \cdot \mathbf{e}_{1}\right]\left[\left(\mathbf{x}-\mathbf{x}_{i}\right) \cdot \mathbf{e}_{\mathbf{2}}\right],
\end{aligned}
$$


where $\mathbf{e}_{1}:=\mathbf{n}\left(\overline{\mathbf{x}}_{i}\right), \mathbf{e}_{2}:=\mathbf{p}\left(\overline{\mathbf{x}}_{i}\right)$ for $\mathbf{x}_{i} \in \Omega_{N \delta}$ and $\mathbf{e}_{1}:=(1,0), \mathbf{e}_{2}:=(0,1)$ when $\mathbf{x}_{i} \in \Omega / \Omega_{N \delta}$. For $\mathbf{x}_{i} \in \Omega / \Omega_{N \delta}$, one may obtain the following formula for $\varpi_{h}$ in light of (1.1).

$$
-2 \tilde{u} D P\left(P^{\mathrm{T}} D P\right)^{-1} \int_{B\left(\mathbf{x}_{i}, \delta\right)} J_{\delta}\left(\left|\mathbf{y}-\mathbf{x}_{\mathbf{i}}\right|\right)\left(R(\mathbf{y})-R\left(\mathbf{x}_{i}\right)\right) \mathrm{d} \mathbf{y}=f\left(\mathbf{x}_{i}\right) .
$$

Similarly, for $\mathbf{x}_{i} \in \Omega_{N \delta}$, we apply the Neumann boundary treatment and obtain the following formula for $\varpi_{h}$ in light of $(2.3)$.

$$
\begin{aligned}
&-2 \tilde{u} D P\left(P^{\mathrm{T}} D P\right)^{-1} \int_{B\left(\mathbf{x}_{i}, \delta\right) \cap \Omega} J_{\delta}\left(\left|\mathbf{y}-\mathbf{x}_{i}\right|\right)\left(R(\mathbf{y})-R\left(\mathbf{x}_{i}\right)\right) \mathrm{d} \mathbf{y} \\
&-2 \tilde{u} D P\left(P^{\mathrm{T}} D P\right)^{-1} M_{\delta}\left(\mathbf{x}_{i}\right) \int_{-\delta}^{\delta} H_{\delta}(|l|)\left(R\left(\mathbf{x}_{l}\right)-R\left(\mathbf{x}_{i}\right)\right) \mathrm{d} \mathbf{x}_{l} \\
&= f\left(\mathbf{x}_{i}\right)+\left(2 \int_{B\left(\mathbf{x}_{i}, \delta\right) \backslash \Omega} J_{\delta}\left(\left|\mathbf{y}-\mathbf{x}_{i}\right|\right)\left(\mathbf{y}-\mathbf{x}_{i}\right) \cdot \mathbf{n}\left(\overline{\mathbf{x}}_{i}\right) \mathrm{d} \mathbf{y}-M_{\delta}\left(\mathbf{x}_{i}\right) \kappa\left(\overline{\mathbf{x}}_{i}\right)\right) g\left(\overline{\mathbf{x}}_{i}\right) \\
&-\int_{B\left(\mathbf{x}_{i}, \delta\right) \backslash \Omega} J_{\delta}\left(\left|\mathbf{y}-\mathbf{x}_{i}\right|\right)\left[\left|\left(\mathbf{y}-\overline{\mathbf{x}}_{i}\right) \cdot \mathbf{n}\left(\overline{\mathbf{x}}_{i}\right)\right|^{2}-\left|\left(\mathbf{x}_{i}-\overline{\mathbf{x}}_{i}\right) \cdot \mathbf{n}\left(\overline{\mathbf{x}}_{i}\right)\right|^{2}\right] \mathrm{d} \mathbf{y} f\left(\mathbf{x}_{i}\right) .
\end{aligned}
$$

For $\mathbf{x} \in \partial \Omega_{D \delta}$, we apply the Dirichlet boundary condition and therefore $u_{\delta}(\mathbf{x})$ is given. We can then solve for $\tilde{u}$ with (5.8) and (5.9).

Numerically, the problem now reduces to how to integrate quadratic polynomials over $B\left(\mathbf{x}_{i}, \delta\right) \cap \Omega$ and $B\left(\mathbf{x}_{i}, \delta\right) \backslash \Omega$ properly. On simple geometries the integral in (5.8) and (5.9) can be calculated analytically, while for more generalized cases where the boundary curve is more complicated, an analytic quadrature is intractable. We note that when $\delta$ is sufficiently small, $B\left(\mathbf{x}_{i}, \delta\right) \cap \Omega$ and $B\left(\mathbf{x}_{i}, \delta\right) \backslash \Omega$ can be written as the regions between two curves, and one can then evaluate the integral via numerical integration, for instance, with high-order Gaussian quadrature rules.

\section{Numerical Results}

In this section, we present the asymptotic convergence of the proposed boundary treatment by considering the nonlocal diffusion problem on three types of representative domains: a square domain in Section 6.1 which represents the case with 0 curvature on $\partial \Omega_{N}$; a circular domain in Section 6.2, which illustrates a case with constant curvature on $\partial \Omega$; and an elliptical domain in Section 6.3, with varying curvatures along the domain boundary. Here we note that the square domain case does not satisfy the $C^{3}$ regularity requirement and it is therefore outside the scope of the model problem analysis presented earlier. Hence the results in Section 6.1 also demonstrate how robust the convergence rate results are when relaxing the $C^{3}$ assumption on domain regularity. In this paper we focus on the type (3) convergence, i.e., the convergence of numerical solutions to the local solution as $h, \delta$ goes to 0 simultaneously, by testing four different combinations of $(h, \delta) \rightarrow 0$ : (1) fixing $h / \delta=C$, taking $\delta \rightarrow 0$; (2) fixing $h / \sqrt{\delta}=C$, taking $\delta \rightarrow 0$; (3) fixing $h / \delta^{2}=C$, taking $\delta \rightarrow 0$; and (4) decreasing $\delta$ with a fixed $h \ll \delta$. Here we note that in combination type (2), one has to keep sufficiently large ratio between $\delta$ and $h$ so as to make sure that the optimization problem (5.4) in the meshfree quadrature rule is well-posed. For combination type (4) tests, we aim to investigate the convergence of analytical nonlocal solutions $u_{\delta}$ to the local limit $u_{0}$, by keeping a sufficiently small fixed $h$ such that $h \ll \min \delta$ in the tests. Therefore, the numerical errors in combination type (4) tests are sufficiently small comparing with the differences between local and nonlocal solutions, and results indicate the convergence of analytical nonlocal solution $u_{\delta}$ to the local limit $u_{0}$ with a decreasing $\delta$. However, here we note that for a fixed $h$ one can not take $\delta \rightarrow 0$ in combination type (4) tests.

\subsection{Test 1: curvature $\kappa(\mathrm{x})=0$}

In this numerical example, we demonstrate a case where the Neumann boundary is a line segment. Specifically, we take the computational domain as $\Omega=[0,1] \times[0,1]$, with $\partial \Omega_{N}=\{(1, y): y \in[0,1]\}$ and $\partial \Omega_{D}=\partial \Omega \backslash \partial \Omega_{N}$. 
TABle 1. Test 1: convergence to the local solution for the $\kappa(\mathbf{x})=0$ case with fixed $\delta=C h$. Here the convergence order is with respect to $\delta$.

\begin{tabular}{lllllllll}
\hline \hline \multirow{2}{*}{$h$} & \multicolumn{9}{c}{$\delta / h=4$} & \multicolumn{4}{c}{$\delta / h=3.5$} \\
\cline { 2 - 8 } & $\left\|u_{\delta}-u_{0}\right\|_{\infty}$ & Order & $\left\|u_{\delta}-u_{0}\right\|_{2}$ & Order & $\left\|u_{\delta}-u_{0}\right\|_{\infty}$ & Order & $\left\|u_{\delta}-u_{0}\right\|_{2}$ & Order \\
\hline $2^{-3}$ & $1.45 \times 10^{-1}$ & - & $3.06 \times 10^{-2}$ & - & $9.04 \times 10^{-2}$ & - & $3.01 \times 10^{-2}$ & - \\
$2^{-4}$ & $2.34 \times 10^{-2}$ & 2.63 & $5.80 \times 10^{-3}$ & 2.39 & $1.37 \times 10^{-2}$ & 2.72 & $5.40 \times 10^{-3}$ & 2.48 \\
$2^{-5}$ & $4.25 \times 10^{-3}$ & 2.38 & $1.30 \times 10^{-3}$ & 2.16 & $2.50 \times 10^{-3}$ & 2.45 & $1.10 \times 10^{-3}$ & 2.30 \\
$2^{-6}$ & $1.00 \times 10^{-3}$ & 2.17 & $3.02 \times 10^{-4}$ & 2.11 & $5.65 \times 10^{-4}$ & 2.15 & $2.68 \times 10^{-4}$ & 2.04 \\
$2^{-7}$ & $2.48 \times 10^{-4}$ & 2.01 & $7.38 \times 10^{-5}$ & 2.03 & $1.34 \times 10^{-4}$ & 2.07 & $6.53 \times 10^{-5}$ & 2.04 \\
\hline
\end{tabular}

TABle 2. Test 1: convergence to the local solution for the $\kappa(\mathbf{x})=0$ case with fixed $\delta=C h^{2}$ and $\delta=C \sqrt{h}$. Here the convergence order is with respect to $\delta$.

\begin{tabular}{llllllllllll}
\hline \hline \multicolumn{1}{c}{$\sqrt{\delta} / h=2^{9 / 2}$} & \multicolumn{7}{c}{$\delta^{2} / h=2^{-2}$} \\
\hline$h$ & $\delta$ & $\left\|u_{\delta}-u_{0}\right\|_{\infty}$ & Order & $\left\|u_{\delta}-u_{0}\right\|_{2}$ & Order & $h$ & $\delta$ & $\left\|u_{\delta}-u_{0}\right\|_{\infty}$ & Order & $\left\|u_{\delta}-u_{0}\right\|_{2}$ & Order \\
\hline $2^{-5}$ & $2^{-1}$ & $5.79 \times 10^{-1}$ & - & $5.86 \times 10^{-2}$ & - & $2^{-5}$ & $2^{-7 / 2}$ & $3.30 \times 10^{-3}$ & - & $1.60 \times 10^{-3}$ & - \\
$2^{-6}$ & $2^{-3}$ & $4.90 \times 10^{-3}$ & 3.44 & $1.60 \times 10^{-3}$ & 2.60 & $2^{-6}$ & $2^{-4}$ & $1.50 \times 10^{-3}$ & 2.27 & $7.39 \times 10^{-4}$ & 2.24 \\
$2^{-7}$ & $2^{-5}$ & $1.76 \times 10^{-4}$ & 2.40 & $9.05 \times 10^{-5}$ & 2.07 & $2^{-7}$ & $2^{-9 / 2}$ & $7.54 \times 10^{-4}$ & 1.98 & $3.70 \times 10^{-4}$ & 2.00 \\
\hline
\end{tabular}

TABLE 3 . Test 1: convergence to the local solution for the $\kappa(\mathbf{x})=0$ case with decreasing $\delta$ and fixed $h \ll \delta$. Here the convergence order is with respect to $\delta$.

\begin{tabular}{llllll}
\hline \hline$h$ & $\delta$ & $\left\|u_{\delta}-u_{0}\right\|_{\infty}$ & Order & $\left\|u_{\delta}-u_{0}\right\|_{2}$ & Order \\
\hline $2^{-7}$ & $2^{-2}$ & $2.25 \times 10^{-1}$ & - & $1.18 \times 10^{-2}$ & - \\
$2^{-7}$ & $2^{-3}$ & $1.15 \times 10^{-2}$ & 4.29 & $1.60 \times 10^{-3}$ & 2.88 \\
$2^{-7}$ & $2^{-4}$ & $7.54 \times 10^{-4}$ & 3.93 & $3.70 \times 10^{-4}$ & 2.11 \\
$2^{-7}$ & $2^{-5}$ & $1.76 \times 10^{-4}$ & 2.10 & $9.05 \times 10^{-5}$ & 2.03 \\
\hline
\end{tabular}

The local limit of the nonlocal problem has a smooth analytical solution $u_{0}(x, y)=\sin (\pi x) \cos (\pi y)$, together with $f(x, y)=2 \pi^{2} \sin (\pi x) \cos (\pi y)$ and $\left.\frac{\partial u}{\partial \mathbf{n}}\right|_{x=1}=g(y)=-\pi \cos (\pi y)$. We apply the analytical local solution as a Dirichlet boundary condition over $\partial \Omega_{D \delta}$ by letting $u_{\delta}=u_{0}$, and impose the Neumann-type constraint (2.3) over the region $\Omega_{N \delta}=[1-\delta, 1] \times[0,1]$. With uniform discretization of mesh size $h$, we demonstrate the difference between the numerical results and $u_{0}$ in the $L^{\infty}$-norm and $L^{2}$-norm, and the convergence order with respect to $\delta$ in Tables 1-3. It is observed that as $\delta$ decreases, the numerical solution from the proposed nonlocal Neumann-type constraint problem converges to the local analytical solution $u_{0}$ as $O\left(\delta^{2}\right)$, which therefore verifies the analysis in Section 4 and demonstrates the asymptotic compatibility of the numerical solver.

\subsection{Test 2: constant curvature $\kappa(\mathrm{x})$}

We now consider as domain the unit circle $\Omega=\left\{(x, y) \mid x^{2}+y^{2} \leq 1\right\}, \partial \Omega_{N}=\partial \Omega$ and with the value $u_{\delta}(0,-1)=u_{0}(0,-1)$ given to make the problem well-posed. Similarly as in test 1 , we consider a smooth local solution $u_{0}(x, y)=\sin (\pi x) \cos (\pi y)$, with $f(x, y)=2 \pi^{2} \sin (\pi x) \cos (\pi y)$ and $\left.\frac{\partial u}{\partial \mathbf{n}}\right|_{(x, y) \in \partial \Omega_{N}}=g(x, y)=$ $\pi x \cos (\pi x) \cos (\pi y)-\pi y \sin (\pi x) \sin (\pi y)$, with uniform discretization of mesh-size $h$. The $L^{\infty}$-norm and $L^{2}$-norm convergence results are presented in Tables 4-6. It can be observed that the convergence rate is $O\left(\delta^{2}\right)$ as $\delta$ decreases, consistent with the analysis in Section 4. 
TABLE 4 . Test 2: convergence to the local solution for the $\kappa(\mathbf{x})=$ const case with fixed $\delta=C h$. Here the convergence order is with respect to $\delta$.

\begin{tabular}{lllllllll}
\hline \hline \multirow{2}{*}{$h$} & \multicolumn{9}{c}{$\delta / h=4$} & \multicolumn{4}{c}{$\delta / h=3.5$} \\
\cline { 2 - 8 } & $\left\|u_{\delta}-u_{0}\right\|_{\infty}$ & Order & $\left\|u_{\delta}-u_{0}\right\|_{2}$ & Order & $\left\|u_{\delta}-u_{0}\right\|_{\infty}$ & Order & $\left\|u_{\delta}-u_{0}\right\|_{2}$ & Order \\
\hline $2^{-3}$ & $3.74 \times 10^{-1}$ & - & $2.13 \times 10^{-1}$ & - & $2.98 \times 10^{-1}$ & - & $1.77 \times 10^{-1}$ & - \\
$2^{-4}$ & $1.10 \times 10^{-1}$ & 1.77 & $6.88 \times 10^{-2}$ & 1.63 & $8.21 \times 10^{-2}$ & 1.86 & $5.17 \times 10^{-2}$ & 1.78 \\
$2^{-5}$ & $2.68 \times 10^{-2}$ & 2.04 & $1.68 \times 10^{-2}$ & 2.03 & $1.98 \times 10^{-2}$ & 2.05 & $1.24 \times 10^{-2}$ & 2.06 \\
$2^{-6}$ & $6.30 \times 10^{-3}$ & 2.09 & $3.90 \times 10^{-3}$ & 2.11 & $4.70 \times 10^{-3}$ & 2.07 & $2.90 \times 10^{-3}$ & 2.10 \\
$2^{-7}$ & $1.50 \times 10^{-4}$ & 2.07 & $9.37 \times 10^{-4}$ & 2.06 & $1.10 \times 10^{-3}$ & 2.10 & $6.91 \times 10^{-4}$ & 2.07 \\
\hline
\end{tabular}

TABLE 5. Test 2: convergence to the local solution for the $\kappa(\mathbf{x})=$ const case with fixed $\delta=C h^{2}$ and $\delta=C \sqrt{h}$. Here the convergence order is with respect to $\delta$.

\begin{tabular}{|c|c|c|c|c|c|c|c|c|c|c|c|}
\hline \multicolumn{6}{|c|}{$\sqrt{\delta} / h=4 \sqrt{14}$} & \multicolumn{6}{|c|}{$\delta^{2} / h=2.45$} \\
\hline$h$ & $\delta$ & $\left\|u_{\delta}-u_{0}\right\|_{\infty}$ & Order & $\left\|u_{\delta}-u_{0}\right\|_{2}$ & Order & $h$ & $\delta$ & $\left\|u_{\delta}-u_{0}\right\|_{\infty}$ & Order & $\left\|u_{\delta}-u_{0}\right\|_{2}$ & Order \\
\hline $2^{-4}$ & $7 / 2^{3}$ & 2.14 & - & $4.47 \times 10^{-1}$ & - & $1 / 5$ & $7 / 10$ & 1.0569 & - & $6.50 \times 10^{-1}$ & - \\
\hline $2^{-5}$ & $7 / 2^{5}$ & $9.08 \times 10^{-2}$ & 2.19 & $5.67 \times 10^{-2}$ & 1.58 & $1 / 20$ & $7 / 20$ & $2.86 \times 10^{-1}$ & 1.87 & $1.51 \times 10^{-1}$ & 2.11 \\
\hline $2^{-6}$ & $7 / 2^{7}$ & $4.70 \times 10^{-3}$ & 2.10 & $2.90 \times 10^{-3}$ & 2.10 & $1 / 80$ & $7 / 40$ & $7.16 \times 10^{-2}$ & 2.00 & $3.83 \times 10^{-2}$ & 1.98 \\
\hline
\end{tabular}

TABLE 6 . Test 2: convergence to the local solution for the $\kappa(\mathbf{x})=$ const case with decreasing $\delta$ and fixed $h \ll \delta$. Here the convergence order is with respect to $\delta$.

\begin{tabular}{llllll}
\hline \hline$h$ & $\delta$ & $\left\|u_{\delta}-u_{0}\right\|_{\infty}$ & Order & $\left\|u_{\delta}-u_{0}\right\|_{2}$ & Order \\
\hline $2^{-6}$ & $2^{-1}$ & $6.57 \times 10^{-1}$ & - & $2.38 \times 10^{-1}$ & - \\
$2^{-6}$ & $2^{-2}$ & $1.27 \times 10^{-1}$ & 2.38 & $7.52 \times 10^{-2}$ & 1.66 \\
$2^{-6}$ & $2^{-3}$ & $2.87 \times 10^{-2}$ & 2.14 & $1.79 \times 10^{-2}$ & 2.07 \\
$2^{-6}$ & $2^{-4}$ & $6.40 \times 10^{-3}$ & 2.16 & $4.00 \times 10^{-3}$ & 2.16 \\
\hline
\end{tabular}

\subsection{Test 3: non-constant curvature $\kappa(\mathrm{x})$}

In our previous two tests, the problem domains have either zero curvature or a constant curvature on the Neumann boundary. In this section, we further consider a more generalized domain with a non-constant curvature on its boundary. We consider the ellipse $\Omega=\left\{(x, y) \mid x^{2} / 4+y^{2} \leq 1\right\}$ with $\left.\partial \Omega_{N}=\partial \Omega\right\}$. $u_{\delta}(0,-1)=u_{0}(0,-1)$ is given to guarantee the compatibility condition. Here we note that when $\delta<1 / 2$, the orthogonal projection $\overline{\mathbf{x}}$ is well-defined for any $\mathbf{x} \in \Omega_{N \delta}$. We again consider a smooth local solution $u_{0}(x, y)=\sin (\pi x) \cos (\pi y)$ with $f(x, y)=2 \pi^{2} \sin (\pi x) \cos (\pi y)$, and we demonstrate the convergence of the numerical solution to the local solution with mesh-size $h$. As shown in Tables 7-9, second order convergence of $\delta$ is achieved which therefore verifies the estimates in Section 4 and illustrates the asymptotic compatibility for a domain with nonuniform boundary curvature.

Moreover, we note that in the cases with constant curvature boundary, the Neumann-type constraint problem gives the analytical solution $u_{\delta}=u_{0}$ for the patch test problem with a linear solution $u_{0}(x, y)=x+y$. Therefore, in the previous two tests, the numerical solver passes the linear patch test with machine precision. In the elliptical domain with non-constant curvature, we further investigate the linear patch test problem, and the numerical results are illustrated in Table 10. It can be observed that although the numerical solution is no longer within 
TABLE 7 . Test 3: convergence to the local solution for the non-constant $\kappa(\mathbf{x})$ case with fixed $\delta=C h$. Here the convergence order is with respect to $\delta$.

\begin{tabular}{llllllllll}
\hline \hline \multirow{2}{*}{$h$} & \multicolumn{9}{c}{$\delta / h=4$} \\
\cline { 2 - 9 } & $\left\|u_{\delta}-u_{0}\right\|_{\infty}$ & Order & $\left\|u_{\delta}-u_{0}\right\|_{2}$ & Order & $\left\|u_{\delta}-u_{0}\right\|_{\infty}$ & Order & $\left\|u_{\delta}-u_{0}\right\|_{2}$ & Order \\
\hline $2^{-3}$ & $4.80 \times 10^{-3}$ & - & $2.40 \times 10^{-3}$ & - & $3.60 \times 10^{-3}$ & - & $1.90 \times 10^{-3}$ & - \\
$2^{-4}$ & $2.80 \times 10^{-3}$ & 0.78 & $1.60 \times 10^{-3}$ & 0.59 & $2.20 \times 10^{-3}$ & 0.71 & $1.20 \times 10^{-2}$ & 0.66 \\
$2^{-5}$ & $9.34 \times 10^{-4}$ & 1.58 & $5.39 \times 10^{-4}$ & 1.57 & $7.00 \times 10^{-4}$ & 1.65 & $3.99 \times 10^{-4}$ & 1.59 \\
$2^{-6}$ & $2.33 \times 10^{-4}$ & 2.00 & $1.25 \times 10^{-4}$ & 2.11 & $1.88 \times 10^{-4}$ & 1.91 & $1.07 \times 10^{-4}$ & 1.90 \\
\hline
\end{tabular}

TABLE 8. Test 3: convergence to the local solution for the non-constant $\kappa(\mathbf{x})$ case with fixed $\delta=C h^{2}$ and $\delta=C \sqrt{h}$. Here the convergence order is with respect to $\delta$.

\begin{tabular}{llllllllllll}
\hline \hline \multicolumn{9}{c}{$\sqrt{\delta} / h=16$} & \multicolumn{6}{c}{$\delta^{2} / h=1.8$} \\
\hline$h$ & $\delta$ & $\left\|u_{\delta}-u_{0}\right\|_{\infty}$ & Order & $\left\|u_{\delta}-u_{0}\right\|_{2}$ & Order & $h$ & $\delta$ & $\left\|u_{\delta}-u_{0}\right\|_{\infty}$ & Order & $\left\|u_{\delta}-u_{0}\right\|_{2}$ & Order \\
\hline $2^{-4}$ & $2^{0}$ & $4.00 \times 10^{-1}$ & - & $5.34 \times 10^{-2}$ & - & $1 / 20$ & $3 / 10$ & $4.60 \times 10^{-3}$ & - & $2.40 \times 10^{-3}$ & - \\
$2^{-5}$ & $2^{-2}$ & $3.20 \times 10^{-3}$ & 3.48 & $1.80 \times 10^{-3}$ & 2.45 & $1 / 60$ & $\sqrt{3} / 10$ & $1.80 \times 10^{-3}$ & 1.71 & $9.83 \times 10^{-4}$ & 1.63 \\
$2^{-6}$ & $2^{-4}$ & $2.33 \times 10^{-4}$ & 1.89 & $1.25 \times 10^{-4}$ & 1.92 & $1 / 80$ & $3 / 20$ & $1.36 \times 10^{-3}$ & 1.95 & $7.16 \times 10^{-4}$ & 2.20 \\
\hline
\end{tabular}

TABLE 9. Test 3: convergence to the local solution for the non-constant $\kappa(\mathbf{x})$ case with decreasing $\delta$ and fixed $h \ll \delta$. Here the convergence order is with respect to $\delta$.

\begin{tabular}{lllll}
\hline$\delta$ & $\left\|u_{\delta}-u_{0}\right\|_{\infty}$ & Order & $\left\|u_{\delta}-u_{0}\right\|_{2_{2}}$ & Order \\
\hline $3.5 / 8$ & $4.12 \times 10$ & - & $1.48 \times 10^{2}$ & - \\
$3.5 / 16$ & $9.72 \times 10^{-3}$ & 5.40 & $1.20 \times 10^{-3}$ & 3.63 \\
$3.5 / 32$ & $7.44 \times 10^{-4}$ & 3.71 & $4.26 \times 10^{-4}$ & 1.50 \\
$3.5 / 64$ & $1.88 \times 10^{-4}$ & 1.98 & $1.07 \times 10^{-4}$ & 1.99 \\
\hline
\end{tabular}

TABLE 10. Test 3: linear patch test for convergence to the local solution for the non-constant $\kappa(\mathbf{x})$ case.

\begin{tabular}{lllllllll}
\hline \hline \multirow{2}{*}{$h$} & \multicolumn{9}{c}{$\delta / h=4$} & \multicolumn{5}{c}{$\delta / h=3.5$} \\
\cline { 2 - 9 } & $\left\|u_{\delta}-u_{0}\right\|_{\infty}$ & order & $\left\|u_{\delta}-u_{0}\right\|_{2}$ & order & $\left\|u_{\delta}-u_{0}\right\|_{\infty}$ & order & $\left\|u_{\delta}-u_{0}\right\|_{2}$ & order \\
\hline $2^{-3}$ & $1.71 \times 10^{-1}$ & - & $7.87 \times 10^{-2}$ & - & $1.14 \times 10^{-1}$ & - & $5.94 \times 10^{-2}$ & - \\
$2^{-4}$ & $2.89 \times 10^{-2}$ & 2.57 & $1.55 \times 10^{-2}$ & 2.34 & $2.16 \times 10^{-2}$ & 2.39 & $1.16 \times 10^{-2}$ & 2.36 \\
$2^{-5}$ & $6.01 \times 10^{-3}$ & 2.27 & $3.20 \times 10^{-3}$ & 2.28 & $4.50 \times 10^{-3}$ & 2.26 & $2.40 \times 10^{-3}$ & 2.27 \\
$2^{-6}$ & $1.20 \times 10^{-3}$ & 2.32 & $6.04 \times 10^{-4}$ & 2.40 & $8.35 \times 10^{-4}$ & 2.43 & $4.11 \times 10^{-4}$ & 2.55 \\
$2^{-7}$ & $1.26 \times 10^{-4}$ & 3.25 & $4.69 \times 10^{-5}$ & 3.69 & $1.39 \times 10^{-4}$ & 2.58 & $6.20 \times 10^{-5}$ & 2.72 \\
\hline
\end{tabular}

machine precision accuracy, the numerical solution converges to the analytical solution with an $O\left(h^{2}\right)$ rate as $h \rightarrow 0$.

\section{EXTENSION: DOMAIN WITH CORNERS}

In many popular nonlocal problem applications, it is common that the Neumann-type boundary contains corners. For example, on a peridynamic problem with damage, once a crack initiates and bifurcates, new zigzag 


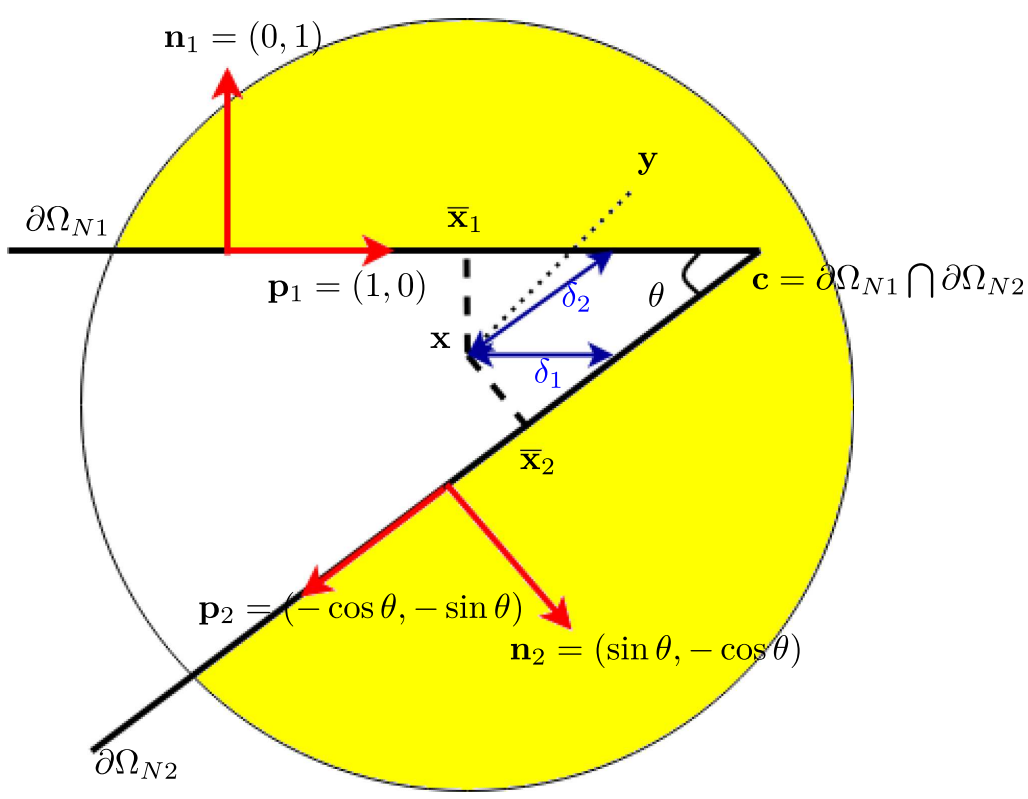

FiguRE 6. Geometric assumptions and notation for the corner case. Here the yellow region denotes $B(\mathbf{x}, \delta) \cap \partial \Omega_{N \delta}$.

boundary forms and the Neumann-type boundary condition must be applied on these new boundaries. To investigate how well the new Neumann-type constraint formulation extrapolates to the setting of Lipschitz domains, in this section we further extend the proposed formulation to boundaries with corners. We also numerically show the performance as well as asymptotic compatibility properties on a sample test problem with Neumann-type boundary on two sides of a square domain. Specifically, in Section 7.1 we derive the formulation near a corner by approximating $-2 \int_{\partial \Omega_{N \delta}} J_{\delta}(\mathbf{x}-\mathbf{y})(u(\mathbf{y})-u(\mathbf{x}))$ dy. Then in Section 7.2 we firstly adopt a similar problem domain as in test 1 of Section 6.1 but with Neumann-type boundary conditions applied on two sides of the boundary including their intersecting corner. To further demonstrate the performance on complicated domains, we also test the formulation on a cross-shape domain which is non-convex (as shown in Fig. 7) with Neumann-type boundary conditions applied on all boundaries. In both cases we demonstrate the second-order convergence of the nonlocal solution to the corresponding local limit as $h, \delta \rightarrow 0$.

\subsection{Flux condition and numerical setting}

In this section, we extend the numerical algorithm to a domains with corners. For simplicity, here we assume that there are two boundaries with Neumann-type boundary conditions:

$$
\begin{array}{ll}
\frac{\partial u}{\partial \mathbf{n}_{1}}=g_{1}, & \text { on } \partial \Omega_{N 1}, \\
\frac{\partial u}{\partial \mathbf{n}_{2}}=g_{2}, & \text { on } \partial \Omega_{N 2},
\end{array}
$$

and the two boundaries intersect at $\mathbf{c}=\partial \Omega_{N 1} \bigcap \partial \Omega_{N 2}$. For any point $\mathbf{x}$ satisfying $|\mathbf{x}-\mathbf{c}|<\delta$, we project $\mathbf{x}$ onto the two boundaries respectively, i.e., $\mathbf{x}=\overline{\mathbf{x}}_{1}-s_{x 1} \mathbf{n}_{1}\left(\overline{\mathbf{x}}_{1}\right)=\overline{\mathbf{x}}_{2}-s_{x 2} \mathbf{n}_{2}\left(\overline{\mathbf{x}}_{2}\right)$. In this section, we assume that both $\partial \Omega_{N 1}$ and $\partial \Omega_{N 2}$ are straight lines near the corner $\mathbf{c}$, although the formulation can be further extended to more general cases. Denote $\theta$ as the angle between $\partial \Omega_{N_{1}}$ and $\partial \Omega_{N_{2}}$, without loss of generality we further denote $\mathbf{n}_{1}=(0,1)$ and $\mathbf{n}_{2}=(\sin \theta,-\cos \theta)$. Correspondingly, we have $\mathbf{p}_{1}=(1,0)$ and $\mathbf{p}_{2}=(-\cos \theta,-\sin \theta)$. We 


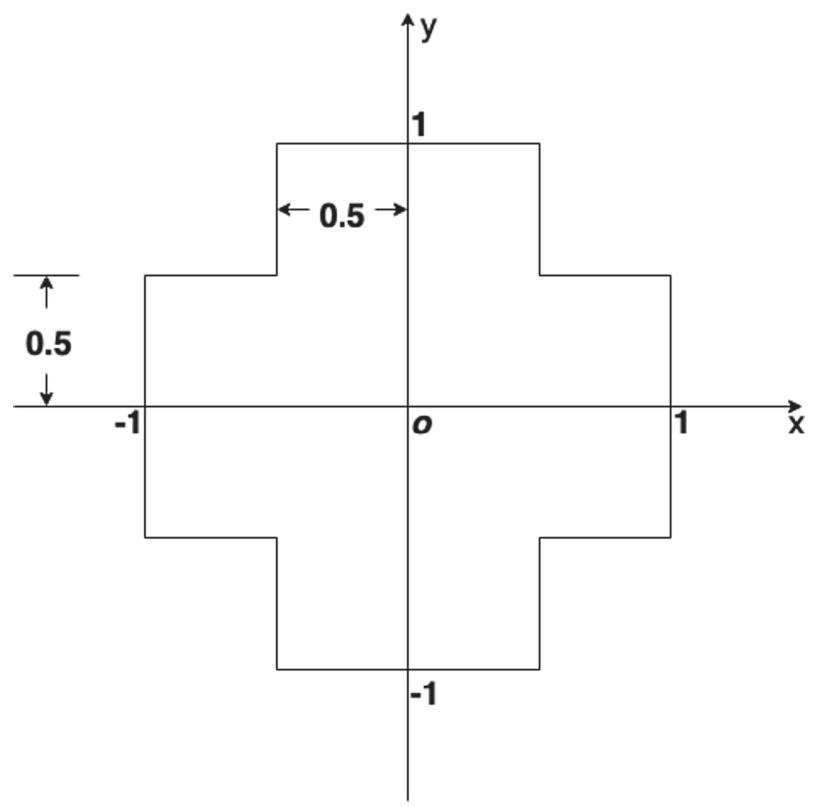

Figure 7. A cross-shape non-convex domain employed in the second test of corner formulation.

illustrate geometric assumptions and notation in Figure 6. For each point $\mathbf{x}=\left(x_{1}, x_{2}\right)$, with Taylor expansion we have the following approximation for $u(\mathbf{y})-u(\mathbf{x})$ with $\mathbf{y}=\left(y_{1}, y_{2}\right) \in B(\mathbf{x}, \delta) \cap \partial \Omega_{N \delta}$ :

$$
\begin{aligned}
u(\mathbf{y})-u(\mathbf{x})= & d_{1} \frac{\partial u(\mathbf{x})}{\partial \mathbf{n}_{1}}+d_{2} \frac{\partial u(\mathbf{x})}{\partial \mathbf{n}_{2}}+\frac{1}{2} d_{1}^{2}[u(\mathbf{x})]_{n_{1} n_{1}}+\frac{1}{2} d_{2}^{2}[u(\mathbf{x})]_{n_{2} n_{2}}+d_{1} d_{2}[u(\mathbf{x})]_{n_{1} n_{2}}+O\left(\delta^{3}\right) \\
= & d_{1} g_{1}\left(\overline{\mathbf{x}}_{1}\right)+d_{2} g_{2}\left(\overline{\mathbf{x}}_{2}\right)+\left(\frac{1}{2} d_{1}^{2}-\left(\overline{\mathbf{x}}_{1}-\mathbf{x}\right) \cdot \mathbf{n}_{1} d_{1}\right)\left(-f(\mathbf{x})-[u(\mathbf{x})]_{p_{1} p_{1}}\right) \\
& +\left(\frac{1}{2} d_{2}^{2}-\left(\overline{\mathbf{x}}_{2}-\mathbf{x}\right) \cdot \mathbf{n}_{2} d_{2}\right)\left(-f(\mathbf{x})-[u(\mathbf{x})]_{p_{2} p_{2}}\right) \\
& +\frac{1}{2 \sin \theta} d_{1} d_{2}\left(\frac{\partial g_{1}\left(\overline{\mathbf{x}}_{1}\right)}{\partial \mathbf{p}_{1}}-\frac{\partial g_{2}\left(\overline{\mathbf{x}}_{2}\right)}{\partial \mathbf{p}_{2}}+f(\mathbf{x}) \sin \theta \cos \theta\right)+O\left(\delta^{3}\right)
\end{aligned}
$$

where

Moreover, we have

$$
d_{1}:=\frac{\cos \theta}{\sin \theta}\left(y_{1}-x_{1}\right)+\left(y_{2}-x_{2}\right), \quad d_{2}:=\frac{1}{\sin \theta}\left(y_{1}-x_{1}\right) .
$$

$$
[u(\mathbf{x})]_{p_{1} p_{1}}+[u(\mathbf{x})]_{p_{2} p_{2}}=-f(\mathbf{x})+\cot \theta \frac{\partial g_{1}\left(\overline{\mathbf{x}}_{1}\right)}{\partial \mathbf{p}_{1}}-\cot \theta \frac{\partial g_{1}\left(\overline{\mathbf{x}}_{2}\right)}{\partial \mathbf{p}_{2}}+O(\delta) .
$$

Let

$$
\begin{aligned}
& D_{1}=2 \int_{\partial \Omega_{N \delta}} J_{\delta}(|\mathbf{x}-\mathbf{y}|)\left[\frac{1}{2} d_{1}^{2}-\left(\overline{\mathbf{x}}_{1}-\mathbf{x}\right) \cdot \mathbf{n}_{1} d_{1}\right] \mathrm{d} \mathbf{y}, \\
& D_{2}=2 \int_{\partial \Omega_{N \delta}} J_{\delta}(|\mathbf{x}-\mathbf{y}|)\left[\frac{1}{2} d_{2}^{2}-\left(\overline{\mathbf{x}}_{2}-\mathbf{x}\right) \cdot \mathbf{n}_{2} d_{2}\right] \mathrm{d} \mathbf{y},
\end{aligned}
$$

substituting the above approximations into the nonlocal formulation and neglecting the higher order terms give the algorithm. For $D_{1}>D_{2}$, we take $\delta_{1}$ as the arc length from $\mathbf{x}$ to $\partial \Omega_{N}$ following the contour parallel to $\partial \Omega_{N 1}$ 
and use $2 \int_{-\delta_{1}}^{\delta_{1}} H_{\delta_{1}}(|l|)\left(u\left(\mathbf{x}_{l 1}\right)-u(\mathbf{x})\right) \mathrm{d} \mathbf{x}_{l 1}$ to denote the integral on this contour which approximates $[u(\mathbf{x})]_{p_{1} p_{1}}$ :

$$
\begin{aligned}
& -2 \int_{\Omega} J_{\delta}(|\mathbf{x}-\mathbf{y}|)(u(\mathbf{y})-u(\mathbf{x})) \mathrm{d} \mathbf{y}+4\left(D_{1}-D_{2}\right) \int_{-\delta_{1}}^{\delta_{1}} H_{\delta_{1}}(|l|)\left(u\left(\mathbf{x}_{l 1}\right)-u(\mathbf{x})\right) \mathrm{d} \mathbf{x}_{l 1} \\
& =f(\mathbf{x})-D_{1} f(\mathbf{x})-D_{2} \cot \theta\left(\frac{\partial g_{1}\left(\overline{\mathbf{x}}_{1}\right)}{\partial \mathbf{p}_{1}}-\frac{\partial g_{2}\left(\overline{\mathbf{x}}_{2}\right)}{\partial \mathbf{p}_{2}}\right)+2 \int_{\partial \Omega_{N \delta}} J_{\delta}(|\mathbf{x}-\mathbf{y}|)\left(d_{1} g_{1}\left(\overline{\mathbf{x}}_{1}\right)\right. \\
& \left.\quad+d_{2} g_{2}\left(\overline{\mathbf{x}}_{2}\right)+\frac{1}{2 \sin \theta} d_{1} d_{2}\left(\frac{\partial g_{1}\left(\overline{\mathbf{x}}_{1}\right)}{\partial \mathbf{p}_{1}}-\frac{\partial g_{2}\left(\overline{\mathbf{x}}_{2}\right)}{\partial \mathbf{p}_{2}}+f(\mathbf{x}) \sin \theta \cos \theta\right)\right) \mathrm{d} \mathbf{y} .
\end{aligned}
$$

Else, we similarly take $\delta_{2}$ as the arc length from $\mathbf{x}$ to $\partial \Omega_{N}$ following the contour parallel to $\partial \Omega_{N 2}$ and use $2 \int_{-\delta_{2}}^{\delta_{2}} H_{\delta_{2}}(|l|)\left(u\left(\mathbf{x}_{l 2}\right)-u(\mathbf{x})\right) \mathrm{d} \mathbf{x}_{l 2}$ to denote the integral on this contour which approximates $[u(\mathbf{x})]_{p_{2} p_{2}}$ :

$$
\begin{aligned}
& -2 \int_{\Omega} J_{\delta}(|\mathbf{x}-\mathbf{y}|)(u(\mathbf{y})-u(\mathbf{x})) \mathrm{d} \mathbf{y}+4\left(D_{2}-D_{1}\right) \int_{-\delta_{2}}^{\delta_{2}} H_{\delta_{2}}(|l|)\left(u\left(\mathbf{x}_{l 2}\right)-u(\mathbf{x})\right) \mathrm{d} \mathbf{x}_{l 2} \\
& = \\
& \quad f(\mathbf{x})-D_{2} f(\mathbf{x})-D_{1} \cot \theta\left(\frac{\partial g_{1}\left(\overline{\mathbf{x}}_{1}\right)}{\partial \mathbf{p}_{1}}-\frac{\partial g_{2}\left(\overline{\mathbf{x}}_{2}\right)}{\partial \mathbf{p}_{2}}\right)+2 \int_{\partial \Omega_{N \delta}} J_{\delta}(|\mathbf{x}-\mathbf{y}|)\left(d_{1} g_{1}\left(\overline{\mathbf{x}}_{1}\right)\right. \\
& \left.\quad+d_{2} g_{2}\left(\overline{\mathbf{x}}_{2}\right)+\frac{1}{2 \sin \theta} d_{1} d_{2}\left(\frac{\partial g_{1}\left(\overline{\mathbf{x}}_{1}\right)}{\partial \mathbf{p}_{1}}-\frac{\partial g_{2}\left(\overline{\mathbf{x}}_{2}\right)}{\partial \mathbf{p}_{2}}+f(\mathbf{x}) \sin \theta \cos \theta\right)\right) \mathrm{d} \mathbf{y} .
\end{aligned}
$$

Here we note that in (7.3) and (7.4), since the coefficient for nonlocal Laplace Beltrami $\int_{-\delta_{i}}^{\delta_{i}} H_{\delta_{i}}(|l|)\left(u\left(\mathbf{x}_{l i}\right)-\right.$ $u(\mathbf{x})) \mathrm{d} \mathbf{x}_{l i}(i=1$ or 2$)$ is not negative, the coercivity is not guaranteed in this formulation. However, numerical experiments in Section 7.2 still suggest that the method remains robust in practice, possibly due to the fact that the corner formulation only impacts the discretization points within distance $\delta$ to both edges. Moreover, here we note that when considering a sufficiently smooth function $u$ in a finite domain $\Omega$, one has

$$
\int_{\Omega} \int_{\Omega} J_{\delta}(|\mathbf{x}-\mathbf{y}|)[u(\mathbf{y})-u(\mathbf{x})]^{2} \mathrm{~d} \mathbf{y} \mathrm{d} \mathbf{x}=O(1), \quad \int_{\Omega_{\delta}} M_{\delta}(\mathbf{x}) \int_{-\delta}^{\delta} H_{\delta}(|l|)\left[u\left(\mathbf{x}_{l}\right)-u(\mathbf{x})\right]^{2} \mathrm{~d} \mathbf{x}_{l} \mathrm{~d} \mathbf{x}=O(\delta) .
$$

Therefore, in the nonlocal weak formulation the $-2 \int_{\Omega} J_{\delta}(|\mathbf{x}-\mathbf{y}|)(u(\mathbf{y})-u(\mathbf{x}))$ dy term gets more dominant as $\delta \rightarrow 0$, which possibly explains the numerical robustness of the corner formulation (7.3) and (7.4).

\subsection{Numerical results}

In this section, we firstly investigate the numerical performance of formulation (7.3) and (7.4) on a square domain $\Omega=[0,1] \times[0,1]$ with Neumann-type boundary conditions applied on $\partial \Omega_{N 1}=\{(1, y): y \in[0,1]\}$ and $\partial \Omega_{N 2}=\{(x, 1): x \in[0,1]\}$. Note that the Neumann-type boundary contains a corner $\mathbf{c}=(1,1)$ where the numerical algorithms (7.3) and (7.4) are employed. We set the analytical local solution as $u_{0}(x, y)=x^{2} y^{2}$, which then yields $f(x, y)=-2\left(x^{2}+y^{2}\right),\left.\frac{\partial u}{\partial \mathbf{n}}\right|_{x=1}=g_{1}(y)=2 y^{2}$ and $\left.\frac{\partial u}{\partial \mathbf{n}}\right|_{y=1}=g_{2}(x)=2 x^{2}$. The Dirichlettype condition $u=u_{0}$ is provided in a layer $\partial \Omega_{D \delta}=\{(x, y) \mid(x, y) \in[-\delta, 1] \times[-\delta, 1] / \Omega\}$. With mesh-sizes $h=\{1 / 16,1 / 32,1 / 64,1 / 128\}$ and a fixed ratio $\delta / h=\{4.0,3.5\}$, the numerical results are shown in Table 11, illustrating an $O\left(\delta^{2}\right)$ convergence rate to the local limit.

In the second test of this section, we further consider a non-convex domain with corners. The domain is presented in Figure 7. A Neumann-type boundary condition is applied everywhere over the boundary except for on point $(-1,0)$ where Dirichlet boundary condition is applied to make the problem well posed. We use the analytical local solution $u_{0}(x, y)=\sin (x) \cos (y)$ with $f(x, y)=2 \sin (x) \cos (y)$, and keep the ration $\delta / h=3.5$ while decreasing $\delta$ and $h$ simultaneously. The numerical results are provided in Table 12, which shows a secondorder convergence rate in $\delta$. This example shows that the proposed formulation is capable to handle non-convex domains consisting of line segments and corners as boundaries. 
TABLE 11. Convergence to the local solution in the square domain with Neumann-type boundary including a corner.

\begin{tabular}{lllllllll}
\hline \hline \multirow{2}{*}{$h$} & \multicolumn{9}{c}{$\delta / h=4$} & \multicolumn{5}{c}{$\delta / h=3.5$} \\
\cline { 2 - 9 } & $\left\|u_{\delta}-u_{0}\right\|_{\infty}$ & Order & $\left\|u_{\delta}-u_{0}\right\|_{2}$ & Order & $\left\|u_{\delta}-u_{0}\right\|_{\infty}$ & Order & $\left\|u_{\delta}-u_{0}\right\|_{2}$ & Order \\
\hline $2^{-3}$ & $7.43 \times 10^{-2}$ & - & $1.91 \times 10^{-2}$ & - & $5.45 \times 10^{-2}$ & - & $1.46 \times 10^{-2}$ & - \\
$2^{-4}$ & $1.52 \times 10^{-2}$ & 2.29 & $4.01 \times 10^{-3}$ & 2.26 & $1.13 \times 10^{-2}$ & 2.27 & $3.10 \times 10^{-3}$ & 2.24 \\
$2^{-5}$ & $3.30 \times 10^{-3}$ & 2.20 & $9.12 \times 10^{-4}$ & 2.13 & $2.40 \times 10^{-3}$ & 2.24 & $6.97 \times 10^{-4}$ & 2.15 \\
$2^{-6}$ & $7.42 \times 10^{-3}$ & 2.15 & $2.17 \times 10^{-4}$ & 2.06 & $5.60 \times 10^{-4}$ & 2.11 & $1.66 \times 10^{-4}$ & 2.07 \\
$2^{-7}$ & $1.74 \times 10^{-4}$ & 2.09 & $5.32 \times 10^{-5}$ & 2.03 & $1.31 \times 10^{-4}$ & 2.09 & $4.04 \times 10^{-5}$ & 2.03 \\
\hline
\end{tabular}

TABLE 12. Convergence to the local solution on a non-convex cross-shape domain.

\begin{tabular}{lllll}
\hline \hline \multirow{2}{*}{$h$} & \multicolumn{4}{c}{$\delta / h=3.5$} \\
\cline { 2 - 5 } & $\left\|u_{\delta}-u_{0}\right\|_{\infty}$ & Order & $\left\|u_{\delta}-u_{0}\right\|_{2}$ & Order \\
\hline $1 / 40$ & $7.75 \times 10^{-4}$ & - & $2.80 \times 10^{-3}$ & - \\
$1 / 80$ & $1.73 \times 10^{-4}$ & 2.16 & $4.66 \times 10^{-4}$ & 2.59 \\
$1 / 160$ & $2.84 \times 10^{-5}$ & 2.60 & $8.11 \times 10^{-5}$ & 2.52 \\
\hline
\end{tabular}

\section{CONCLUSion AND FUture WORK}

In this paper we have introduced a new nonlocal Neumann-type constraint for the 2D nonlocal diffusion problem which is an analogue to the local flux boundary condition and for the first time achieved the optimal second-order convergence rate $O\left(\delta^{2}\right)$ to the local limit in the $L^{\infty}(\Omega)$ norm. The formulation is applied on a collar layer inside the domain and therefore requires no mesh or extrapolation outside the problem domain, which enables the possibility of applying the physical boundary conditions on a sharp interface. We have shown that when the problem domain is bounded, convex, connected and possesses sufficient regularity, the proposed nonlocal Neumann-type constraint with the nonlocal diffusion equation is well-posed. The nonlocal solution $u_{\delta}$ converges to the solution $u_{0}$ from the corresponding local problem in the $L^{2}(\Omega)$ norm as the horizon size $\delta \rightarrow 0$. Moreover, when the solution is continuous in $\bar{\Omega}$ and the Neumann type boundary is convex, we have further proved the second-order convergence of $u_{\delta}$ in the $L^{\infty}(\Omega)$ norm. Numerically, we have developed an asymptotically compatible particle method based on a meshfree quadrature rule for the Neumann-type constraint problem. Numerical examples on domains with representative geometries and boundary curvatures were investigated, and the optimal convergence rate $O\left(\delta^{2}\right)$ in the $L^{\infty}(\Omega)$ norm was observed in all instances, verifying the asymptotic compatibility of both the Neumann boundary treatment and discretization. Finally, we have demonstrated that the regularity assumption may be relaxed in practice and the formulation can be extended to non-convex domain with corners, which greatly improves the applicability of the proposed formulation for more complicated scenarios. Although the formulation does not preserve formal coercivity near the corner, numerical experiments indicate that the formulation is robust in practice and achieves the optimal convergence rate to the local limit.

We note that the formulation described in this paper actually provides an approach for applying the Neumanntype boundary condition on general compactly supported nonlocal integro-differential equations (IDEs) with radial kernels. As a natural extension, we are working on a nonlocal trace theorem which will immediately extend the current analysis results in the $L^{2}$ norm to problems with inhomogeneous boundary conditions, and we are also developing a sharp traction boundary condition for peridynamics which is consistent with the classical elasticity theory. 


\section{Appendix A.}

\section{A.1. Proof of Lemma 3.9}

In this section, we aim to provide the detailed proof for Lemma 3.9. Since $B_{\delta}\left(\tilde{u}_{\delta}, v\right)=(f, v)_{L^{2}(\Omega)}$ for any $v \in S_{\delta}$, with Lemmas 3.4-3.6 we have

$$
\left\|\tilde{u}_{\delta}\right\|_{S_{\delta}}^{2} \leq C B_{\delta}\left(\tilde{u}_{\delta}, \tilde{u}_{\delta}\right)=C\left(f, \tilde{u}_{\delta}\right)_{L^{2}(\Omega)} \leq C\|f\|_{L^{2}(\Omega)}\left\|\tilde{u}_{\delta}\right\|_{L^{2}(\Omega)} \leq C\|f\|_{L^{2}(\Omega)}\left\|\tilde{u}_{\delta}\right\|_{S_{\delta}}
$$

which yields the uniform boundedness of $\left\{\tilde{u}_{\delta}\right\}$. With Lemma 3.8, we have the convergence of a subsequence of $\left\{\tilde{u}_{\delta}\right\}$ in $L^{2}(\Omega)$. Here we use the same $\tilde{u}_{\delta}$ to denote the convergent subsequence, then $\tilde{u}_{\delta} \rightarrow u_{*} \in S_{0}$. To prove the lemma, it suffices to show that $u_{*}=u_{0}$ or

$$
B_{0}\left(u_{*}, v\right):=\left(\nabla u_{*}, \nabla v\right)=(f, v)_{L^{2}(\Omega)}, \quad \forall v \in C^{\infty}(\Omega) .
$$

Taking a standard mollifier $\phi_{\epsilon}$ satisfying $\int_{B(0, \epsilon)} \phi_{\epsilon}(\mathbf{x}) \mathrm{d} \mathbf{x}=1$ and letting $\tilde{u}_{\delta, \epsilon}=\int_{B(0, \epsilon)} \tilde{u}_{\delta}(\mathbf{x}-\mathbf{y}) \phi_{\epsilon}(\mathbf{y}) \mathrm{d} \mathbf{y}$, we define $\Omega_{\epsilon}=\{\mathbf{x} \in \Omega: \operatorname{dist}(\mathbf{x}, \partial \Omega)<\epsilon\}$ and $\Omega^{\epsilon}=\{\mathbf{x} \in \Omega: \operatorname{dist}(\mathbf{x}, \partial \Omega) \geq \epsilon\}$. Assuming that $\epsilon>\delta$, for $v \in C^{\infty}(\Omega)$ we denote

$$
\begin{aligned}
& B_{\delta}^{\epsilon}(u, v)=\int_{\Omega^{\epsilon}} \int_{\Omega^{\epsilon}} J_{\delta}(|\mathbf{x}-\mathbf{y}|)(u(\mathbf{y})-u(\mathbf{x}))(v(\mathbf{y})-v(\mathbf{x})) \mathrm{d} \mathbf{y} \mathrm{d} \mathbf{x}, \\
& B_{0}^{\epsilon}(u, v)=\int_{\Omega^{\epsilon}} \nabla u \cdot \nabla v \mathrm{~d} \mathbf{x} .
\end{aligned}
$$

Since

$$
\begin{aligned}
B_{\delta}^{\epsilon}\left(\tilde{u}_{\delta, \epsilon}, v\right) & =\int_{\Omega^{\epsilon}} \int_{\Omega^{\epsilon}} J_{\delta}(|\mathbf{x}-\mathbf{y}|)\left(\tilde{u}_{\delta, \epsilon}(\mathbf{y})-\tilde{u}_{\delta, \epsilon}(\mathbf{x})\right)(v(\mathbf{y})-v(\mathbf{x})) \mathrm{d} \mathbf{y} \mathrm{d} \mathbf{x} \\
& =\int_{\Omega^{\epsilon}} \int_{\Omega^{\epsilon}} J_{\delta}(|\mathbf{x}-\mathbf{y}|)\left(\int_{B(0, \epsilon)} \phi_{\epsilon}(\mathbf{z}) \tilde{u}_{\delta}(\mathbf{y}-\mathbf{z}) \mathrm{d} \mathbf{z}-\int_{B(0, \epsilon)} \phi_{\epsilon}(\mathbf{z}) \tilde{u}_{\delta}(\mathbf{x}-\mathbf{z}) \mathrm{d} \mathbf{z}\right)(v(\mathbf{y})-v(\mathbf{x})) \mathrm{d} \mathbf{y} \mathrm{d} \mathbf{x} \\
& =\int_{B(0, \epsilon)} \phi_{\epsilon}(\mathbf{z})\left(\int_{\Omega^{\epsilon}} \int_{\Omega^{\epsilon}} J_{\delta}(|\mathbf{x}-\mathbf{y}|)\left(\tilde{u}_{\delta}(\mathbf{y}-\mathbf{z})-\tilde{u}_{\delta}(\mathbf{x}-\mathbf{z})\right)(v(\mathbf{y})-v(\mathbf{x})) \mathrm{d} \mathbf{y} \mathrm{d} \mathbf{x}\right) \mathrm{d} \mathbf{z} \\
& =\int_{B(0, \epsilon)} \phi_{\epsilon}(\mathbf{z}) B_{\delta}^{\epsilon}\left(\tilde{u}_{\delta}(\mathbf{x}-\mathbf{z}), v(\mathbf{x})\right) \mathrm{d} \mathbf{z},
\end{aligned}
$$

to show (A.1) it suffices to prove that when $\delta \rightarrow 0$ first then $\epsilon \rightarrow 0$, we have

$$
B_{\delta}^{\epsilon}\left(\tilde{u}_{\delta, \epsilon}, v\right) \rightarrow B_{0}\left(u_{*}, v\right)
$$

and

$$
\int_{B(0, \epsilon)} \phi_{\epsilon}(\mathbf{z}) B_{\delta}^{\epsilon}\left(\tilde{u}_{\delta}(\mathbf{x}-\mathbf{z}), v(\mathbf{x})\right) \mathrm{d} \mathbf{z} \rightarrow(f, v)_{L^{2}(\Omega)}
$$

To show (A.2) we first fix $\epsilon$ and let $\delta \rightarrow 0$. Since $\epsilon>\delta, \Omega^{\epsilon} \cap \Omega_{\delta}=\Phi$ and $\Omega_{\delta} \subset \Omega_{\epsilon}$. Then

$$
\begin{aligned}
B_{\delta}^{\epsilon}\left(\tilde{u}_{\delta, \epsilon}, v\right)= & \int_{\Omega^{\epsilon}} \int_{\Omega} J_{\delta}(|\mathbf{x}-\mathbf{y}|)\left(\tilde{u}_{\delta, \epsilon}(\mathbf{y})-\tilde{u}_{\delta, \epsilon}(\mathbf{x})\right)(v(\mathbf{y})-v(\mathbf{x})) \mathrm{d} \mathbf{y} \mathrm{d} \mathbf{x} \\
& -\int_{\Omega^{\epsilon}} \int_{\Omega_{\epsilon}} J_{\delta}(|\mathbf{x}-\mathbf{y}|)\left(\tilde{u}_{\delta, \epsilon}(\mathbf{y})-\tilde{u}_{\delta, \epsilon}(\mathbf{x})\right)(v(\mathbf{y})-v(\mathbf{x})) \mathrm{d} \mathbf{y} \mathrm{d} \mathbf{x} .
\end{aligned}
$$

Since $\tilde{u}_{\delta, \epsilon} \rightarrow u_{*, \epsilon}$ as $\delta \rightarrow 0$, with Proposition 3.4 of [63] and the Dominated Convergence Theorem,

$$
\lim _{\epsilon \rightarrow 0} \lim _{\delta \rightarrow 0} \int_{\Omega^{\epsilon}} \int_{\Omega} J_{\delta}(|\mathbf{x}-\mathbf{y}|)\left(\tilde{u}_{\delta, \epsilon}(\mathbf{y})-\tilde{u}_{\delta, \epsilon}(\mathbf{x})\right)(v(\mathbf{y})-v(\mathbf{x})) \mathrm{d} \mathbf{y} \mathrm{d} \mathbf{x}=\lim _{\epsilon \rightarrow 0} B_{0}^{\epsilon}\left(u_{*, \epsilon}, v\right)=B_{0}\left(u_{*}, v\right) .
$$


On the other hand, for the second term, with the uniform boundedness

$$
\lim _{\epsilon \rightarrow 0} \lim _{\delta \rightarrow 0}\left|\int_{\Omega^{\epsilon}} \int_{\Omega_{\epsilon}} J_{\delta}(|\mathbf{x}-\mathbf{y}|)\left(\tilde{u}_{\delta, \epsilon}(\mathbf{y})-\tilde{u}_{\delta, \epsilon}(\mathbf{x})\right)(v(\mathbf{y})-v(\mathbf{x})) \mathrm{d} \mathbf{y} \mathrm{d} \mathbf{x}\right| \leq C \lim _{\epsilon \rightarrow 0} \operatorname{area}\left(\Omega_{\epsilon}\right)=0 .
$$

Hence (A.2) has been proved. For (A.3) it suffices to show that

$$
\begin{aligned}
\lim _{\epsilon \rightarrow 0} \lim _{\delta \rightarrow 0}\left|B_{\delta}^{\epsilon}\left(\tilde{u}_{\delta}(\mathbf{x}-\mathbf{z}), v(\mathbf{x})\right)-(f(\mathbf{x}), v(\mathbf{x}))_{L^{2}(\Omega)}\right| \\
\quad=\lim _{\epsilon \rightarrow 0} \lim _{\delta \rightarrow 0}\left|B_{\delta}^{\epsilon}\left(\tilde{u}_{\delta}(\mathbf{x}-\mathbf{z}), v(\mathbf{x})\right)-B_{\delta}\left(\tilde{u}_{\delta}(\mathbf{x}), v(\mathbf{x})\right)\right|=0 .
\end{aligned}
$$

Denote $\Omega^{\mathbf{z} \epsilon}=\left\{\mathbf{x} \in \Omega: \mathbf{x}-\mathbf{z} \in \Omega^{\epsilon}\right\}$, we have

$$
\begin{aligned}
&\left|B_{\delta}^{\epsilon}\left(\tilde{u}_{\delta}(\mathbf{x}-\mathbf{z}), v(\mathbf{x})\right)-B_{\delta}\left(\tilde{u}_{\delta}(\mathbf{x}), v(\mathbf{x})\right)\right| \\
&=\mid \int_{\Omega^{\epsilon}} \int_{\Omega^{\epsilon}} J_{\delta}(|\mathbf{x}-\mathbf{y}|)\left(\tilde{u}_{\delta}(\mathbf{y}-\mathbf{z})-\tilde{u}_{\delta}(\mathbf{x}-\mathbf{z})\right)(v(\mathbf{y})-v(\mathbf{x})) \mathrm{d} \mathbf{y} \mathrm{d} \mathbf{x} \\
&-\int_{\Omega} \int_{\Omega} J_{\delta}(|\mathbf{x}-\mathbf{y}|)\left(\tilde{u}_{\delta}(\mathbf{y})-\tilde{u}_{\delta}(\mathbf{x})\right)(v(\mathbf{y})-v(\mathbf{x})) \mathrm{d} \mathbf{y} \mathrm{d} \mathbf{x} \\
&-\int_{\Omega_{\delta}} M_{\delta}(\mathbf{x}) \int_{-\delta}^{\delta} H_{\delta}(|l|)\left[\tilde{u}^{\delta}\left(\mathbf{x}_{l}\right)-\tilde{u}^{\delta}(\mathbf{x})\right]\left[v\left(\mathbf{x}_{l}\right)-v(\mathbf{x})\right] \mathrm{d} \mathbf{x}_{l} \mathrm{~d} \mathbf{x} \\
&-\int_{\Omega_{\delta}} \int_{-\delta}^{\delta}\left[M_{\delta}\left(\mathbf{x}_{l}\right) \frac{\left|\mathbf{r}^{\prime}(\mathbf{x})\right|}{\left|\mathbf{r}^{\prime}\left(\mathbf{x}_{l}\right)\right|}-M_{\delta}(\mathbf{x})\right] H_{\delta}(|l|)\left[\tilde{u}^{\delta}\left(\mathbf{x}_{l}\right)-\tilde{u}^{\delta}(\mathbf{x})\right] \mathrm{d} \mathbf{x}_{l} v(\mathbf{x}) \mathrm{d} \mathbf{x} \mid \\
& \leq\left|\int_{\Omega^{\mathbf{z} \epsilon}} \int_{\Omega^{\mathbf{z} \epsilon}} J_{\delta}(|\mathbf{x}-\mathbf{y}|)\left(\tilde{u}_{\delta}(\mathbf{y})-\tilde{u}_{\delta}(\mathbf{x})\right)(v(\mathbf{y}+\mathbf{z})-v(\mathbf{x}+\mathbf{z})-v(\mathbf{y})+v(\mathbf{x})) \mathrm{d} \mathbf{y} \mathrm{d} \mathbf{x}\right| \\
&+\mid \int_{\Omega^{\mathbf{z} \epsilon}} \int_{\Omega^{\mathbf{z} \epsilon}} J_{\delta}(|\mathbf{x}-\mathbf{y}|)\left(\tilde{u}_{\delta}(\mathbf{y})-\tilde{u}_{\delta}(\mathbf{x})\right)(v(\mathbf{y})-v(\mathbf{x})) \mathrm{d} \mathbf{y} \mathrm{d} \mathbf{x} \\
&-\int_{\Omega} \int_{\Omega} J_{\delta}(|\mathbf{x}-\mathbf{y}|)\left(\tilde{u}_{\delta}(\mathbf{y})-\tilde{u}_{\delta}(\mathbf{x})\right)(v(\mathbf{y})-v(\mathbf{x})) \mathrm{d} \mathbf{y} \mathrm{d} \mathbf{x} \mid \\
&+\mid \int_{\Omega_{\delta}} M_{\delta}(\mathbf{x}) \int_{-\delta}^{\delta} H_{\delta}(|l|)\left[\tilde{u}^{\delta}\left(\mathbf{x}_{l}\right)-\tilde{u}^{\delta}(\mathbf{x})\right]\left[v\left(\mathbf{x}_{l}\right)-v(\mathbf{x})\right] \mathrm{d} \mathbf{x}_{l} \mathrm{~d} \mathbf{x} \\
&+\int_{\Omega_{\delta}} \int_{-\delta}^{\delta}\left[M_{\delta}\left(\mathbf{x}_{l}\right) \frac{\left|\mathbf{r}^{\prime}(\mathbf{x})\right|}{\left|\mathbf{r}^{\prime}\left(\mathbf{x}_{l}\right)\right|}-M_{\delta}(\mathbf{x})\right] H_{\delta}(|l|)\left[\tilde{u}^{\delta}\left(\mathbf{x}_{l}\right)-\tilde{u}^{\delta}(\mathbf{x})\right] \mathrm{d} \mathbf{x}_{l} v(\mathbf{x}) \mathrm{d} \mathbf{x} \mid \\
&:= \mathrm{I}+\mathrm{II}+\mathrm{III} .
\end{aligned}
$$

For the first term we have

$$
\mathrm{I} \leq\left\|\tilde{u}_{\delta}\right\|_{S_{\delta}}\|v(\mathbf{x}+\mathbf{z})-v(\mathbf{x})\|_{S_{\delta}} \leq\left\|\tilde{u}_{\delta}\right\|_{S_{\delta}}\|v(\mathbf{x}+\mathbf{z})-v(\mathbf{x})\|_{S_{0}}
$$

which goes to 0 as $\epsilon \rightarrow 0$ since $|\mathbf{z}| \leq \epsilon$ and $v \in C^{\infty}(\Omega)$. For the second term, since $|\mathbf{z}|<\epsilon, \Omega \backslash \Omega^{\mathbf{z} \epsilon} \subset \Omega_{2 \epsilon}$. Therefore

$$
\begin{aligned}
\mathrm{II} \leq & \left|\int_{\Omega \backslash \Omega^{\mathbf{z} \epsilon}} \int_{\Omega} J_{\delta}(|\mathbf{x}-\mathbf{y}|)\left(\tilde{u}_{\delta}(\mathbf{y})-\tilde{u}_{\delta}(\mathbf{x})\right)(v(\mathbf{y})-v(\mathbf{x})) \mathrm{d} \mathbf{y} \mathrm{d} \mathbf{x}\right| \\
& +\left|\int_{\Omega \backslash \Omega^{\mathbf{z} \epsilon}} \int_{\Omega^{\mathbf{z} \epsilon}} J_{\delta}(|\mathbf{x}-\mathbf{y}|)\left(\tilde{u}_{\delta}(\mathbf{y})-\tilde{u}_{\delta}(\mathbf{x})\right)(v(\mathbf{y})-v(\mathbf{x})) \mathrm{d} \mathbf{y} \mathrm{d} \mathbf{x}\right|
\end{aligned}
$$




$$
\begin{aligned}
& \leq 2\left\|\tilde{u}_{\delta}\right\|_{S_{\delta}}\left(\int_{\Omega \backslash \Omega^{\mathbf{z} \epsilon}} \int_{\Omega} J_{\delta}(|\mathbf{x}-\mathbf{y}|)(v(\mathbf{y})-v(\mathbf{x}))^{2} \mathrm{~d} \mathbf{y} \mathrm{d} \mathbf{x}\right)^{1 / 2} \\
& \leq 2\left\|\tilde{u}_{\delta}\right\|_{S_{\delta}}\left(\int_{\Omega_{2 \epsilon}} \int_{\Omega} J_{\delta}(|\mathbf{x}-\mathbf{y}|)(v(\mathbf{y})-v(\mathbf{x}))^{2} \mathrm{~d} \mathbf{y} \mathrm{d} \mathbf{x}\right)^{1 / 2}
\end{aligned}
$$

Since $v \in C^{\infty}(\Omega)$, we have $\lim _{\epsilon \rightarrow 0} \lim _{\delta \rightarrow 0} \int_{\Omega_{2 \epsilon}} \int_{\Omega} J_{\delta}(\mathbf{x}-\mathbf{y})(v(\mathbf{y})-v(\mathbf{x}))^{2} \mathrm{~d} \mathbf{y} \mathrm{d} \mathbf{x}=0$ and therefore $\lim _{\epsilon \rightarrow 0} \lim _{\delta \rightarrow 0}$ II $=0$. For the third term we first consider the curvature $\equiv 0$ case. When $\delta$ is sufficiently small, since $M_{\delta}(\mathbf{x}) \leq 3 \pi \sup _{r \leq 1} J(|r|)$ we have

$$
\begin{aligned}
\mathrm{III} & \leq\left|\int_{\Omega_{\delta}} M_{\delta}(\mathbf{x}) \int_{-\delta}^{\delta} H_{\delta}(|l|)\left[\tilde{u}_{\delta}\left(\mathbf{x}_{l}\right)-\tilde{u}_{\delta}(\mathbf{x})\right]\left[v\left(\mathbf{x}_{l}\right)-v(\mathbf{x})\right] \mathrm{d} \mathbf{x}_{l} \mathrm{~d} \mathbf{x}\right| \\
& \leq C\left\|\tilde{u}^{\delta}\right\|_{S_{\delta}}\left(\int_{\Omega_{\delta}} M_{\delta}(\mathbf{x}) \int_{-\delta}^{\delta} H_{\delta}(|l|) l^{2} \mathrm{~d} \mathbf{x}_{l} \sup _{\mathbf{z} \in \Omega_{\delta}}\left|\frac{\partial v(\mathbf{z})}{\partial \mathbf{p}}\right|^{2} \mathrm{~d} \mathbf{x}\right)^{1 / 2} \leq C\left\|\tilde{u}^{\delta}\right\|_{S_{\delta}} \sup _{\mathbf{z} \in \Omega_{\delta}}\left|\frac{\partial v(\mathbf{z})}{\partial \mathbf{p}}\right|\left(\operatorname{area}\left(\Omega_{\delta}\right)\right)^{1 / 2} .
\end{aligned}
$$

Since $v \in C^{\infty}(\Omega), \sup _{\mathbf{z} \in \Omega_{\delta}}\left|\frac{\partial v(\mathbf{z})}{\partial \mathbf{p}}\right| \leq \infty$. Since $\Omega$ is bounded, $\lim _{\delta \rightarrow 0}$ area $\left(\Omega_{\delta}\right)=0$. Hence $\lim _{\epsilon \rightarrow 0} \lim _{\delta \rightarrow 0}$ III $=0$. To prove the case of nonzero curvature, when $\delta$ is sufficiently small (3.19) and (A.6) yield

$$
\begin{aligned}
\mathrm{III} \leq & \left|\int_{\Omega_{\delta}} M_{\delta}(\mathbf{x}) \int_{-\delta}^{\delta} H_{\delta}(|l|)\left[\tilde{u}_{\delta}\left(\mathbf{x}_{l}\right)-\tilde{u}_{\delta}(\mathbf{x})\right]\left[v\left(\mathbf{x}_{l}\right)-v(\mathbf{x})\right] \mathrm{d} \mathbf{x}_{l} \mathrm{~d} \mathbf{x}\right| \\
& +\left|\int_{\Omega_{\delta}} \int_{-\delta}^{\delta}\left[M_{\delta}\left(\mathbf{x}_{l}\right) \frac{\left|\mathbf{r}^{\prime}(\mathbf{x})\right|}{\left|\mathbf{r}^{\prime}(\mathbf{x})_{l}\right|}-M_{\delta}(\mathbf{x})\right] H_{\delta}(|l|)\left[\tilde{u}_{\delta}\left(\mathbf{x}_{l}\right)-\tilde{u}_{\delta}(\mathbf{x})\right] \mathrm{d} \mathbf{x}_{l} v(\mathbf{x}) \mathrm{d} \mathbf{x}\right| \\
\leq & C\left\|\tilde{u}^{\delta}\right\|_{S_{\delta}} \sqrt{\int_{\Omega_{\delta}} v^{2}(\mathbf{x}) \mathrm{d} \mathbf{x}+\sup _{\mathbf{z} \in \Omega_{\delta}}\left|\frac{\partial v(\mathbf{z})}{\partial \mathbf{p}}\right|^{2} \operatorname{area}\left(\Omega_{\delta}\right) \stackrel{\delta \rightarrow 0}{\rightarrow} 0 .}
\end{aligned}
$$

Due to $v \in C^{\infty}(\Omega)$, as $\delta \rightarrow 0 \int_{\Omega_{\delta}} v^{2}(\mathbf{x}) \mathrm{d} \mathbf{x} \rightarrow 0$. Moreover, $\lim _{\delta \rightarrow 0}$ area $\left(\Omega_{\delta}\right)=0$. Therefore $\lim _{\epsilon \rightarrow 0} \lim _{\delta \rightarrow 0} \mathrm{III}=0$ and we have then finished the proof.

\section{A.2. Proof of Lemma 4.2}

In this section, we aim to provide the detailed derivation for Lemma 4.2. For $\mathbf{x} \in \Omega \backslash \Omega_{N \delta}$,

$$
\begin{aligned}
T_{\delta}= & L_{0} u_{0}-L_{\delta} u_{0}=-\triangle u_{0}+2 \int_{\Omega \cup \partial \Omega_{D \delta}} J_{\delta}(|\mathbf{x}-\mathbf{y}|)\left(u_{0}(\mathbf{y})-u_{0}(\mathbf{x})\right) \mathrm{d} \mathbf{y} \\
= & -\triangle u_{0}+\int_{\Omega \cup \partial \Omega_{D \delta}} J_{\delta}(|\mathbf{x}-\mathbf{y}|)\left[u_{0}(\mathbf{x})\right]_{p p}((\mathbf{x}-\mathbf{y}) \cdot \mathbf{p}(\overline{\mathbf{x}}))^{2} \mathrm{~d} \mathbf{y} \\
& +\int_{\Omega \cup \partial \Omega_{D \delta}} J_{\delta}(|\mathbf{x}-\mathbf{y}|)\left[u_{0}(\mathbf{x})\right]_{n n}((\mathbf{x}-\mathbf{y}) \cdot \mathbf{n}(\overline{\mathbf{x}}))^{2} \mathrm{~d} \mathbf{y}+O\left(\delta^{2}\right)=O\left(\delta^{2}\right) .
\end{aligned}
$$

For $\mathbf{x} \in \Omega_{N \delta}$, we will first estimate $\int_{-\delta}^{\delta} H(|l|)\left[u_{0}\left(\mathbf{x}_{l}\right)-u_{0}(\mathbf{x})\right] \mathrm{d} \mathbf{x}_{l}$. With Taylor's expansion we have

$$
\begin{aligned}
u_{0}\left(\mathbf{x}_{l}\right)= & u_{0}(\mathbf{x})+\frac{\partial u_{0}(\mathbf{x})}{\partial \mathbf{n}}\left(\mathbf{x}_{l}-\mathbf{x}\right) \cdot \mathbf{n}(\overline{\mathbf{x}})+\frac{\partial u_{0}(\mathbf{x})}{\partial \mathbf{p}}\left(\mathbf{x}_{l}-\mathbf{x}\right) \cdot \mathbf{p}(\overline{\mathbf{x}}) \\
& +\frac{1}{2}\left[u_{0}(\mathbf{x})\right]_{n n}\left|\left(\mathbf{x}_{l}-\mathbf{x}\right) \cdot \mathbf{n}(\overline{\mathbf{x}})\right|^{2}+\frac{1}{2}\left[u_{0}(\mathbf{x})\right]_{p p}\left|\left(\mathbf{x}_{l}-\mathbf{x}\right) \cdot \mathbf{p}(\overline{\mathbf{x}})\right|^{2}
\end{aligned}
$$




$$
\begin{aligned}
& +\left[u_{0}(\mathbf{x})\right]_{p n}\left(\left(\mathbf{x}_{l}-\mathbf{x}\right) \cdot \mathbf{n}(\overline{\mathbf{x}})\right)\left(\left(\mathbf{x}_{l}-\mathbf{x}\right) \cdot \mathbf{p}(\overline{\mathbf{x}})\right)+\frac{1}{6}\left[u_{0}(\mathbf{x})\right]_{n n n}\left(\left(\mathbf{x}_{l}-\mathbf{x}\right) \cdot \mathbf{n}(\overline{\mathbf{x}})\right)^{3} \\
& +\frac{1}{6}\left[u_{0}(\mathbf{x})\right]_{p p p}\left(\left(\mathbf{x}_{l}-\mathbf{x}\right) \cdot \mathbf{p}(\overline{\mathbf{x}})\right)^{3}+\frac{1}{2}\left[u_{0}(\mathbf{x})\right]_{p n n}\left|\left(\mathbf{x}_{l}-\mathbf{x}\right) \cdot \mathbf{n}(\overline{\mathbf{x}})\right|^{2}\left(\left(\mathbf{x}_{l}-\mathbf{x}\right) \cdot \mathbf{p}(\overline{\mathbf{x}})\right) \\
& +\frac{1}{2}\left[u_{0}(\mathbf{x})\right]_{p p n}\left(\left(\mathbf{x}_{l}-\mathbf{x}\right) \cdot \mathbf{n}(\overline{\mathbf{x}})\right)\left|\left(\mathbf{x}_{l}-\mathbf{x}\right) \cdot \mathbf{p}(\overline{\mathbf{x}})\right|^{2}+O\left(l^{4}\right) .
\end{aligned}
$$

Assuming the boundary $\partial \Omega$ is $C^{3}$ regular, we can approximate $\partial \Omega \cap B(\mathbf{x}, \delta)$ with the osculating circle $\mathcal{C}(\overline{\mathbf{x}})$. When $\partial \Omega$ does not coincide with $\mathcal{C}(\overline{\mathbf{x}})$, we denote $\mathbf{P} \mathbf{x}_{l}$ as the point with distance $l$ to $\mathbf{x}$ along $\mathcal{C}(\overline{\mathbf{x}})$ following the $\mathbf{p}$ direction. For point $\mathbf{x}$, take the Cartesian coordinate system as shown in the right plot of Figure 2 and let $\left(c_{1}(s), c_{2}(s)\right)$ be the curve of boundary $\partial \Omega$ which is parameterized by the arclength $s$. Then we have $\mathbf{x}_{l}=\left(c_{1}(l), c_{2}(l)\right)^{T}$, and

$$
\mathbf{x}_{l}=\mathbf{x}+\left(\begin{array}{l}
l \\
0
\end{array}\right)+\left(\begin{array}{c}
0 \\
\frac{\kappa(\mathbf{x}) l^{2}}{2}
\end{array}\right)+\left(\begin{array}{c}
c_{1}^{\prime \prime \prime}(0) \frac{l^{3}}{6} \\
c_{2}^{\prime \prime \prime}(0) \frac{l^{3}}{6}
\end{array}\right)+O\left(l^{4}\right)
$$

while $\mathbf{P} \mathbf{x}_{l}=\mathbf{x}+\left(\frac{1}{\kappa(\mathbf{x})} \sin (l \kappa(\mathbf{x})), \frac{1}{\kappa(\mathbf{x})}(1-\cos (l \kappa(\mathbf{x})))\right)^{T}$. Therefore

$$
\mathbf{x}_{l}-\mathbf{P} \mathbf{x}_{l}=\left(\begin{array}{c}
\frac{c_{1}^{\prime \prime \prime}(0)+\kappa^{2}(\mathbf{x})}{6} l^{3} \\
\frac{c_{2}^{\prime \prime \prime}(0)}{6} l^{3}
\end{array}\right)+O\left(l^{4}\right) .
$$

With $E_{\delta}$ to denote the region in $A_{\delta}$ which is asymmetric with respect to the $y$ axis in the right plot of Figure 2, we then have the area of $E_{\delta}$ as $\left|E_{\delta}\right| \leq C\left(\delta^{2}-s_{x}^{2}\right)^{2}+O\left(\delta^{5}\right)$. Moreover, adopting the coordinates as shown in the right plot of Figure 2, we have $\left(\mathbf{x}_{l}-\mathbf{x}\right) \cdot \mathbf{n}(\overline{\mathbf{x}})=-\frac{\kappa}{2} l^{2}-\frac{c_{1}^{\prime \prime \prime}(0)}{6} l^{3}+O\left(l^{4}\right),\left(\mathbf{x}_{l}-\mathbf{x}\right) \cdot \mathbf{p}(\overline{\mathbf{x}})=l+\frac{c_{2}^{\prime \prime \prime}(0)}{6} l^{3}+O\left(l^{4}\right)$. Therefore

$$
\begin{aligned}
u_{0}\left(\mathbf{x}_{l}\right)-u_{0}(\mathbf{x})= & -\frac{\partial u_{0}(\mathbf{x})}{\partial \mathbf{n}}\left(\frac{\kappa}{2} l^{2}+\frac{c_{1}^{\prime \prime \prime}(0)}{6} l^{3}\right)+\frac{\partial u_{0}(\mathbf{x})}{\partial \mathbf{p}}\left(l+\frac{c_{2}^{\prime \prime \prime}(0)}{6} l^{3}\right)+\frac{l^{2}}{2}\left[u_{0}(\mathbf{x})\right]_{p p} \\
& -\frac{\kappa l^{3}}{2}\left[u_{0}(\mathbf{x})\right]_{p n}+\frac{l^{3}}{6}\left[u_{0}(\mathbf{x})\right]_{p p p}+O\left(l^{4}\right), \\
u_{0}\left(\mathbf{x}_{-l}\right)-u_{0}(\mathbf{x})= & -\frac{\partial u_{0}(\mathbf{x})}{\partial \mathbf{n}}\left(\frac{\kappa}{2} l^{2}-\frac{c_{1}^{\prime \prime \prime}(0)}{6} l^{3}\right)+\frac{\partial u_{0}(\mathbf{x})}{\partial \mathbf{p}}\left(-l-\frac{c_{2}^{\prime \prime \prime}(0)}{6} l^{3}\right)+\frac{l^{2}}{2}\left[u_{0}(\mathbf{x})\right]_{p p} \\
& +\frac{\kappa l^{3}}{2}\left[u_{0}(\mathbf{x})\right]_{p n}-\frac{l^{3}}{6}\left[u_{0}(\mathbf{x})\right]_{p p p}+O\left(l^{4}\right)
\end{aligned}
$$

which yield

$$
\begin{aligned}
u_{0}\left(\mathbf{x}_{l}\right)+u_{0}\left(\mathbf{x}_{-l}\right)-2 u_{0}(\mathbf{x}) & =\kappa l^{2} \frac{\partial u_{0}(\mathbf{x})}{\partial \mathbf{n}}+l^{2}\left[u_{0}(\mathbf{x})\right]_{p p}+O\left(l^{4}\right) \\
& =\kappa l^{2} \frac{\partial u_{0}(\overline{\mathbf{x}})}{\partial \mathbf{n}}+\kappa l^{2}\left[u_{0}(\mathbf{x})\right]_{n n}((\mathbf{x}-\overline{\mathbf{x}}) \cdot \mathbf{n}(\overline{\mathbf{x}}))+l^{2}\left[u_{0}(\mathbf{x})\right]_{p p}+O\left(l^{4}\right) \\
& =\kappa l^{2}\left[u_{0}(\mathbf{x})\right]_{n n}((\mathbf{x}-\overline{\mathbf{x}}) \cdot \mathbf{n}(\overline{\mathbf{x}}))+l^{2}\left[u_{0}(\mathbf{x})\right]_{p p}+O\left(l^{4}\right) .
\end{aligned}
$$

Therefore

$$
\begin{aligned}
\int_{-\delta}^{\delta} H(|l|)\left[u_{0}\left(\mathbf{x}_{l}\right)-u_{0}(\mathbf{x})\right] \mathrm{d} \mathbf{x}_{l} & =\int_{0}^{\delta} H(|l|)\left[\kappa l^{2}\left[u_{0}(\mathbf{x})\right]_{n n}((\mathbf{x}-\overline{\mathbf{x}}) \cdot \mathbf{n}(\overline{\mathbf{x}}))+l^{2}\left[u_{0}(\mathbf{x})\right]_{p p}+O\left(l^{4}\right)\right] \mathrm{d} \mathbf{x}_{l} \\
& =\frac{\kappa}{2}\left[u_{0}(\mathbf{x})\right]_{n n}((\mathbf{x}-\overline{\mathbf{x}}) \cdot \mathbf{n}(\overline{\mathbf{x}}))+\frac{1}{2}\left[u_{0}(\mathbf{x})\right]_{p p}+O\left(\delta^{2}\right),
\end{aligned}
$$


and

$$
\begin{aligned}
2 M_{\delta}(\mathbf{x}) & \int_{-\delta}^{\delta} H(|l|)\left[u_{0}\left(\mathbf{x}_{l}\right)-u_{0}(\mathbf{x})\right] \mathrm{d} \mathbf{x}_{l} \\
= & M_{\delta}(\mathbf{x})\left[u_{0}(\mathbf{x})\right]_{p p}+\kappa M_{\delta}(\mathbf{x})\left[u_{0}(\mathbf{x})\right]_{n n}((\mathbf{x}-\overline{\mathbf{x}}) \cdot \mathbf{n}(\overline{\mathbf{x}}))+O\left(\delta^{2}\right) \\
= & \kappa M_{\delta}(\mathbf{x})\left[u_{0}(\mathbf{x})\right]_{n n}((\mathbf{x}-\overline{\mathbf{x}}) \cdot \mathbf{n}(\overline{\mathbf{x}}))+\left[u_{0}(\mathbf{x})\right]_{p p} \int_{\partial \Omega_{N \delta}} J_{\delta}(|\mathbf{x}-\mathbf{y}|)|(\mathbf{y}-\mathbf{x}) \cdot \mathbf{p}(\overline{\mathbf{x}})|^{2} \mathrm{~d} \mathbf{y} \\
& -\left[u_{0}(\mathbf{x})\right]_{p p} \int_{\partial \Omega_{N \delta}} J_{\delta}(|\mathbf{x}-\mathbf{y}|)\left(|(\mathbf{y}-\overline{\mathbf{x}}) \cdot \mathbf{n}(\overline{\mathbf{x}})|^{2}-|(\mathbf{x}-\overline{\mathbf{x}}) \cdot \mathbf{n}(\overline{\mathbf{x}})|^{2}\right) \mathrm{d} \mathbf{y}+O\left(\delta^{2}\right) .
\end{aligned}
$$

With the above properties one has the following approximation via Taylor expansion:

$$
\begin{aligned}
2 \int_{\Omega} J_{\delta} & (|\mathbf{x}-\mathbf{y}|)\left(u_{0}(\mathbf{y})-u_{0}(\mathbf{x})\right) \mathrm{d} \mathbf{y} \\
= & -2 \int_{\Omega} J_{\delta}(|\mathbf{x}-\mathbf{y}|)\left[u_{0}(\mathbf{x})\right]_{n n}((\mathbf{x}-\mathbf{y}) \cdot(\overline{\mathbf{x}}-\mathbf{x})) \mathrm{d} \mathbf{y}+2 \int_{E_{\delta}} J_{\delta}(|\mathbf{x}-\mathbf{y}|) \frac{\partial u_{0}(\mathbf{x})}{\partial \mathbf{p}}((\mathbf{x}-\mathbf{y}) \cdot \mathbf{p}(\overline{\mathbf{x}})) \mathrm{d} \mathbf{y} \\
& +\int_{\Omega} J_{\delta}(|\mathbf{x}-\mathbf{y}|)\left[u_{0}(\mathbf{x})\right]_{n n n}((\mathbf{x}-\mathbf{y}) \cdot \mathbf{n}(\overline{\mathbf{x}}))\left(-|\overline{\mathbf{x}}-\mathbf{x}|^{2}+\frac{1}{3}|(\mathbf{x}-\mathbf{y}) \cdot \mathbf{n}(\overline{\mathbf{x}})|^{2}\right) \mathrm{d} \mathbf{y} \\
& +\int_{\Omega} J_{\delta}(|\mathbf{x}-\mathbf{y}|)\left[u_{0}(\mathbf{x})\right]_{n n}|(\mathbf{x}-\mathbf{y}) \cdot \mathbf{n}(\overline{\mathbf{x}})|^{2} \mathrm{~d} \mathbf{y}+\int_{\Omega} J_{\delta}(|\mathbf{x}-\mathbf{y}|)\left[u_{0}(\mathbf{x})\right]_{p p}|(\mathbf{x}-\mathbf{y}) \cdot \mathbf{p}(\overline{\mathbf{x}})|^{2} \mathrm{~d} \mathbf{y} \\
& +\int_{\Omega} J_{\delta}(|\mathbf{x}-\mathbf{y}|)\left[u_{0}(\mathbf{x})\right]_{n p p}((\mathbf{x}-\mathbf{y}) \cdot \mathbf{n}(\overline{\mathbf{x}}))|(\mathbf{x}-\mathbf{y}) \cdot \mathbf{p}(\overline{\mathbf{x}})|^{2} \mathrm{~d} \mathbf{y}+O\left(\delta^{2}\right)
\end{aligned}
$$

and the estimate for $T_{\delta}$ with $\mathbf{x} \in \Omega_{N \delta}$ :

$$
\begin{aligned}
T_{\delta}= & \left(L_{0} u_{0}-L_{N \delta} u_{0}\right)+\left(f_{\delta}-f\right) \\
= & -\triangle u_{0}(\mathbf{x})+2 \int_{\Omega} J_{\delta}(|\mathbf{x}-\mathbf{y}|)\left(u_{0}(\mathbf{y})-u_{0}(\mathbf{x})\right) \mathrm{d} \mathbf{y}+2 M_{\delta}(\mathbf{x}) \int_{-\delta}^{\delta} H(|l|)\left[u_{0}\left(\mathbf{x}_{l}\right)-u_{0}(\mathbf{x})\right] \mathrm{d} \mathbf{x}_{l} \\
& -\int_{\partial \Omega_{N \delta}} J_{\delta}(|\mathbf{x}-\mathbf{y}|)\left(|(\mathbf{y}-\overline{\mathbf{x}}) \cdot \mathbf{n}(\overline{\mathbf{x}})|^{2}-|(\mathbf{x}-\overline{\mathbf{x}}) \cdot \mathbf{n}(\overline{\mathbf{x}})|^{2}\right)\left(-\triangle u_{0}(\mathbf{x})\right) \mathrm{d} \mathbf{y} \\
= & 2 \int_{E_{\delta}} J_{\delta}(|\mathbf{x}-\mathbf{y}|) \frac{\partial u_{0}(\mathbf{x})}{\partial \mathbf{p}}((\mathbf{x}-\mathbf{y}) \cdot \mathbf{p}(\overline{\mathbf{x}})) \mathrm{d} \mathbf{y} \\
& +\int_{\Omega} J_{\delta}(|\mathbf{x}-\mathbf{y}|)\left[u_{0}(\mathbf{x})\right]_{n n n}((\mathbf{x}-\mathbf{y}) \cdot \mathbf{n}(\overline{\mathbf{x}}))\left(-|\overline{\mathbf{x}}-\mathbf{x}|^{2}+\frac{1}{3}|(\mathbf{x}-\mathbf{y}) \cdot \mathbf{n}(\overline{\mathbf{x}})|^{2}\right) \mathrm{d} \mathbf{y} \\
& +\int_{\Omega} J_{\delta}(|\mathbf{x}-\mathbf{y}|)\left[u_{0}(\mathbf{x})\right]_{n p p}((\mathbf{x}-\mathbf{y}) \cdot \mathbf{n}(\overline{\mathbf{x}}))|(\mathbf{x}-\mathbf{y}) \cdot \mathbf{p}(\overline{\mathbf{x}})|^{2} \mathrm{~d} \mathbf{y} \\
& +\kappa M_{\delta}(\mathbf{x})\left[u_{0}(\mathbf{x})\right]_{n n}((\mathbf{x}-\overline{\mathbf{x}}) \cdot \mathbf{n}(\overline{\mathbf{x}}))+O\left(\delta^{2}\right) .
\end{aligned}
$$

We have then finished the proof.

Acknowledgements. The authors want to express their appreciation of the critical suggestions from Dr. Xiaochuan Tian, Dr. Marta D'Elia and Dr. Michael Parks, which improved the clarity and quality of this work. Sandia National Laboratories is a multimission laboratory managed and operated by National Technology and Engineering Solutions of Sandia, LLC., a wholly owned subsidiary of Honeywell International, Inc., for the U.S. 555 Department of Energys National Nuclear Security Administration under contract DE-NA-0003525. H. You and Y. Yu would like to acknowledge support from the National Science Foundation under awards DMS 1753031. Y. Yu is also partially supported by the Lehigh faculty research grant. X.Y. Lu acknowledges the partial support of Lakehead University internal grants 10-50-16422410 and 10-50-16422409, and NSERC Discovery Grant 10-50-16420120. 


\section{REFERENCES}

[1] B. Aksoylu and T. Mengesha, Results on nonlocal boundary value problems. Numer. Funct. Anal. Optim. 31 (2010) $1301-1317$.

[2] B. Alali and M. Gunzburger, Peridynamics and material interfaces. J. Elast. 120 (2015) 225-248.

[3] X. Antoine and H. Barucq, Approximation by generalized impedance boundary conditions of a transmission problem in acoustic scattering. ESAIM:M2AN 39 (2005) 1041-1059.

[4] E. Askari, J. Xu and S. Silling, Peridynamic analysis of damage and failure in composites. In: 44th AIAA Aerospace Sciences Meeting and Exhibit, Reno, Nevada. AIAA, Reston, VA (2006).

[5] M. Astorino, F. Chouly and M.A. Fernández, Robin based semi-implicit coupling in fluid-structure interaction: stability analysis and numerics. SIAM J. Sci. Comput. 31 (2009) 4041-4065.

[6] S. Badia, F. Nobile and C. Vergara, Fluid-structure partitioned procedures based on Robin transmission conditions. J. Comput. Phys. 227 (2008) 7027-7051.

[7] B. Baeumer, M. Kovács, M.M. Meerschaert and H. Sankaranarayanan, Boundary conditions for fractional diffusion. J. Comput. Appl. Math. 336 (2018) 408-424.

[8] G. Barles, C. Georgelin and E.R. Jakobsen, On Neumann and oblique derivatives boundary conditions for nonlocal elliptic equations. J. Differ. Equ. 256 (2014) 1368-1394.

[9] Z.P. Bažant and M. Jirásek, Nonlocal integral formulations of plasticity and damage: survey of progress. J. Eng. Mech. 128 (2002) 1119-1149.

[10] M.A. Bessa, J.T. Foster, T. Belytschko and W.K. Liu, A meshfree unification: reproducing kernel peridynamics. Comput. Mech. 53 (2014) 1251-1264.

[11] F. Bobaru and Y.D. Ha, Adaptive refinement and multiscale modeling in 2D peridynamics. Int. J. Multiscale Comput. Eng. 9 (2011).

[12] J.P. Borthagaray, W. Li and R.H. Nochetto, Finite element discretizations of nonlocal minimal graphs: convergence. Preprint: arXiv: 1905.06395 (2019).

[13] J. Bourgain, H. Brezis and P. Mironescu, Another look at sobolev spaces. hal-00747692 (2001).

[14] S. Brenner and R. Scott, The Mathematical Theory of Finite Element Methods. In Vol. 15. Springer Science \& Business Media (2007).

[15] C. Bucur and E. Valdinoci, Nonlocal Diffusion and Applications. In Vol. 20. Springer (2016).

[16] N. Burch and R. Lehoucq, Classical, nonlocal, and fractional diffusion equations on bounded domains. Int. J. Multiscale Comput. Eng. 9 (2011).

[17] F.A. Chiarello and P. Goatin, Global entropy weak solutions for general non-local traffic flow models with anisotropic kernel. ESAIM: M2AN $\mathbf{5 2}$ (2018) 163-180.

[18] C. Cortazar, M. Elgueta, J.D. Rossi and N. Wolanski, Boundary fluxes for nonlocal diffusion. J. Differ. Equ. 234 (2007) 360-390.

[19] C. Cortazar, M. Elgueta, J.D. Rossi and N. Wolanski, How to approximate the heat equation with Neumann boundary conditions by nonlocal diffusion problems. Arch. Ration. Mech. Anal. 187 (2008) 137-156.

[20] K. Dayal and K. Bhattacharya, A real-space non-local phase-field model of ferroelectric domain patterns in complex geometries. Acta Mater. 55 (2007) 1907-1917.

[21] O. Defterli, M. D'Elia, Q. Du, M. Gunzburger, R. Lehoucq and M.M. Meerschaert, Fractional diffusion on bounded domains. Fractional Calculus Appl. Anal. 18 (2015) 342-360.

[22] P. Demmie and S. Silling, An approach to modeling extreme loading of structures using peridynamics. J. Mech. Mater. Struct. 2 (2007) 1921-1945.

[23] S. Dipierro, X. Ros Oton and E. Valdinoci, Nonlocal problems with Neumann boundary conditions. Rev. Matematica Iberoamericana 33 (2017) 377-416.

[24] S. Dipierro, O. Savin and E. Valdinoci, Boundary behavior of nonlocal minimal surfaces. J. Funct. Anal. 272 (2017) $1791-1851$.

[25] Q. Du and R. Lipton, Peridynamics, fracture, and nonlocal continuum models. SIAM News 47 (2014) 138178758.

[26] Q. Du and K. Zhou, Mathematical analysis for the peridynamic nonlocal continuum theory. ESAIM: M2AN 45 (2011) 217-234.

[27] Q. Du, M. Gunzburger, R.B. Lehoucq and K. Zhou, Analysis and approximation of nonlocal diffusion problems with volume constraints. SIAM Rev. 54 (2012) 667-696.

[28] Q. Du, M. Gunzburger, R.B. Lehoucq and K. Zhou, A nonlocal vector calculus, nonlocal volume-constrained problems, and nonlocal balance laws. Math. Models Methods Appl. Sci. 23 (2013) 493-540.

[29] Q. Du, Z. Huang and R.B. Lehoucq, Nonlocal convection-diffusion volume-constrained problems and jump processes. Discrete Continuous Dyn. Syst.-Ser. B 19 (2014) 373.

[30] Q. Du, R.B. Lehoucq and A.M. Tartakovsky, Integral approximations to classical diffusion and smoothed particle hydrodynamics. Comput. Methods Appl. Mech. Engi. 286 (2015) 216-229.

[31] Q. Du, R. Lipton and T. Mengesha, Multiscale analysis of linear evolution equations with applications to nonlocal models for heterogeneous media. ESAIM:M2AN 50 (2016) 1425-1455.

[32] Q. Du, Y. Tao and X. Tian, A peridynamic model of fracture mechanics with bond-breaking. J. Elast. 132 (2018) 197-218.

[33] E. Emmrich and D. Puhst, Survey of existence results in nonlinear peridynamics in comparison with local elastodynamics. Comput. Methods App. Math. 15 (2015) 483-496. 
[34] E. Emmrich and O. Weckner, Analysis and numerical approximation of an integro-differential equation modeling non-local effects in linear elasticity. Math. Mech. Solids 12 (2007) 363-384.

[35] E. Emmrich and O. Weckner, On the well-posedness of the linear peridynamic model and its convergence towards the navier equation of linear elasticity. Commun. Math. Sci. 5 (2007) 851-864.

[36] H.A. Erbay, S. Erbay and A. Erkip, Convergence of a semi-discrete numerical method for a class of nonlocal nonlinear wave equations. ESAIM:M2AN 52 (2018) 803-826.

[37] J.T. Foster, Dynamic crack initiation toughness: Experiments and peridynamic modeling. Ph.D. thesis, Purdue University (2009).

[38] W. Gerstle, N. Sau and S. Silling, Peridynamic modeling of concrete structures. Nucl. Eng. Des. 237 (2007) $1250-1258$.

[39] G. Grubb, Local and nonlocal boundary conditions for $\mu$-transmission and fractional elliptic pseudodifferential operators. Anal. PDE 7 (2014) 1649-1682.

[40] Y.D. Ha and F. Bobaru, Characteristics of dynamic brittle fracture captured with peridynamics. Eng. Fract. Mech. 78 (2011) 1156-1168.

[41] J.F. Kelly, H. Sankaranarayanan and M.M. Meerschaert, Boundary conditions for two-sided fractional diffusion. J. Comput. Phys. 376 (2019) 1089-1107.

[42] A. Lischke, G. Pang, M. Gulian, F. Song, C. Glusa, X. Zheng, Z. Mao, W. Cai, M.M. Meerschaert, M. Ainsworth and G. Em Karniadakis, What is the fractional laplacian? Preprint arXiv:1801.09767 (2018).

[43] D.J. Littlewood, S.A. Silling, J.A. Mitchell, P.D. Seleson, S.D. Bond, M.L. Parks, D.Z. Turner, D.J. Burnett, J. Ostien and M. Gunzburger, Strong Coupling for Integrated Fracture Modeling. Technical report, Sandia National Laboratories (SNL-NM), Albuquerque, NM (United States); Sandia National Laboratories, Livermore, CA (United States) (2015).

[44] R. Lipton, Dynamic brittle fracture as a small horizon limit of peridynamics. J. Elast. 117 (2014) 21-50.

[45] E. Madenci and E. Oterkus, Peridynamic Theory and Its Applications. Springer (2016).

[46] E. Madenci, M. Dorduncu, A. Barut and N. Phan, Weak form of peridynamics for nonlocal essential and natural boundary conditions. Comput. Methods Appl. Mech. Eng. 337 (2018) 598-631.

[47] R.L. Magin, Fractional Calculus in Bioengineering. Begell House Publishers Inc., Redding, CT (2006).

[48] F. Mainardi, Fractional Calculus and Waves in Linear Viscoelasticity: An Introduction to Mathematical Models. World Scientific (2010).

[49] C. Mantegazza and A.C. Mennucci, Hamilton-Jacobi equations and distance functions on riemannian manifolds. Appl. Math. Optim. 47 (2003) 1-25.

[50] T. Mengesha and Q. Du, Analysis of a scalar peridynamic model with a sign changing kernel. Disc. Cont. Dyn. Sys. B 18 (2013) 1415-1437.

[51] T. Mengesha and Q. Du, Nonlocal constrained value problems for a linear peridynamic navier equation. J. Elast. 116 (2014) $27-51$.

[52] T. Mengesha and Q. Du, Characterization of function spaces of vector fields and an application in nonlinear peridynamics. Nonlinear Anal. 140 (2016) 82-111.

[53] E. Montefusco, B. Pellacci and G. Verzini, Fractional diffusion with Neumann boundary conditions: the logistic equation. Discrete Continuous Dyn. Syst.-Ser. B 18 (2013).

[54] M.L. Parks, P. Seleson, S.J. Plimpton, R.B. Lehoucq and S.A. Silling, Peridynamics with Lammps: A User Guide v0.2 Beta. Sandia National Laboraties (2008).

[55] I. Podlubny, Fractional Differential Equations. An Introduction to Fractional Derivatives, Fractional Differential Equations, to Methods of Their Solution and Some of Their Applications. In Vol. 198. Academic Press (1998).

[56] A.C. Ponce, An estimate in the spirit of poincaré's inequality. J. Eur. Math. Soc. 6 (2004) 1-15.

[57] J. Ren, Z.-Z. Sun and X. Zhao, Compact difference scheme for the fractional sub-diffusion equation with Neumann boundary conditions. J. Comput. Phys. 232 (2013) 456-467.

[58] E.W. Sachs and M. Schu, A priori error estimates for reduced order models in finance. ESAIM:M2AN 47 (2013) 449-469.

[59] P. Seleson, M. Gunzburger and M.L. Parks, Interface problems in nonlocal diffusion and sharp transitions between local and nonlocal domains. Comput. Methods Appl. Mech. Eng. 266 (2013) 185-204.

[60] S.A. Silling, Reformulation of elasticity theory for discontinuities and long-range forces. J. Mech. Phys. Solids 48 (2000) 175-209.

[61] Y. Tao, X. Tian and Q. Du, Nonlocal diffusion and peridynamic models with Neumann type constraints and their numerical approximations. Appl. Math. Comput. 305 (2017) 282-298.

[62] M. Taylor and D.J. Steigmann, A two-dimensional peridynamic model for thin plates. Math. Mech. Solids 20 (2015) 998-1010.

[63] X. Tian and Q. Du, Asymptotically compatible schemes and applications to robust discretization of nonlocal models. SIAM J. Numer. Anal. 52 (2014) 1641-1665.

[64] N. Trask, H. You, Y. Yu and M.L. Parks, An asymptotically compatible meshfree quadrature rule for nonlocal problems with applications to peridynamics. Comput. Methods Appl. Mech. Eng. 343 (2019) 151-165.

[65] O. Weckner, A. Askari, J. Xu, H. Razi and S.A. Silling, Damage and failure analysis based on peridynamics - theory and applications. In: 48th AIAA Structures, Structural Dynamics, and Materials Conf (2007). 
[66] H. Wendland, Scattered Data Approximation. Cambridge University Press 17 (2004).

[67] J. Xu, A. Askari, O. Weckner and S. Silling, Peridynamic analysis of impact damage in composite laminates. J. Aerosp. Eng. 21 (2008) 187-194.

[68] Y. Yu, F. Bargos, H. You, M.L. Parks, M.L. Bittencourt and G.E. Karniadakis, A partitioned coupling framework for peridynamics and classical theory: Analysis and simulations. Comput. Methods Appl. Mech. Eng. 340 (2018) 903-931.

[69] K. Zhou and Q. Du, Mathematical and numerical analysis of linear peridynamic models with nonlocal boundary conditions. SIAM J. Numer. Anal. 48 (2010) 1759-1780.

[70] M. Zimmermann, A Continuum Theory with Long-range Forces for Solids. Ph.D. thesis, Massachusetts Institute of Technology (2005). 